\title{
Design, Development, Implementation, and On-orbit Performance of the Dynamic Ionosphere CubeSat Experiment Mission
}

\section{C.S. Fish • C.M. Swenson • G. Crowley • A. Barjatya • T. Neilsen · J. Gunther •}

I. Azeem • M. Pilinski • R. Wilder · D. Allen • M. Anderson · B. Bingham •

K. Bradford · S. Burr · R. Burt • B. Byers · J. Cook · K. Davis · C. Frazier · S. Grover •

G. Hansen - S. Jensen - R. LeBaron · J. Martineau • J. Miller • J. Nelsen - W. Nelson •

P. Patterson - E. Stromberg · J. Tran · S. Wassom • C. Weston · M. Whiteley •

Q. Young · J. Petersen · S. Schaire · C.R. Davis · M. Bokaie · R. Fullmer • R. Baktur •

J. Sojka $\cdot$ M. Cousins

Received: 29 March 2013 / Accepted: 10 January 2014 / Published online: 6 February 2014

(C) The Author(s) 2014. This article is published with open access at Springerlink.com

\begin{abstract}
Funded by the NSF CubeSat and NASA ELaNa programs, the Dynamic Ionosphere CubeSat Experiment (DICE) mission consists of two 1.5U CubeSats which were launched into an eccentric low Earth orbit on October 28, 2011. Each identical spacecraft carries two Langmuir probes to measure ionospheric in-situ plasma densities, electric field
\end{abstract}

C.S. Fish $(\varangle)$

Space Dynamics Laboratory, Utah State University Research Foundation, 1695 North Research

Park Way, North Logan, UT 84341, USA

e-mail: chad.fish@sdl.usu.edu

C.S. Fish

Center for Space Engineering, Utah State University, Logan, UT, USA

C.S. Fish

Electrical and Computer Engineering Department, Virginia Tech, Blacksburg, VA, USA

C.M. Swenson · J. Gunther · R. Fullmer · R. Baktur · J. Sojka

Utah State University, 4100 Old Main Hill, Logan, UT, USA

G. Crowley $\cdot$ I. Azeem · M. Pilinski · R. Wilder

Atmospheric and Space Technology Research Associates, LLC, 5777 Central Ave., Ste. 221, Boulder, CO, USA

A. Barjatya
Embry-Riddle Aeronautical University, 600 S. Clyde Morris Blvd., Daytona Beach, FL, USA

T. Neilsen · D. Allen · M. Anderson · B. Bingham · K. Bradford · S. Burr · R. Burt · B. Byers · J. Cook ·

K. Davis · C. Frazier · S. Grover · G. Hansen · S. Jensen · R. LeBaron · J. Martineau · J. Miller ·

J. Nelsen · W. Nelson · P. Patterson · E. Stromberg · J. Tran · S. Wassom · C. Weston · M. Whiteley ·

Q. Young

Space Dynamics Laboratory, North Logan, UT, USA 
probes to measure in-situ DC and AC electric fields, and a science grade magnetometer to measure in-situ DC and AC magnetic fields. Given the tight integration of these multiple sensors with the CubeSat platforms, each of the DICE spacecraft is effectively a "sensorsat" capable of comprehensive ionospheric diagnostics. The use of two identical sensor-sats at slightly different orbiting velocities in nearly identical orbits permits the de-convolution of spatial and temporal ambiguities in the observations of the ionosphere from a moving platform. In addition to demonstrating nanosat-based constellation science, the DICE mission is advancing a number of groundbreaking CubeSat technologies including miniaturized mechanisms and high-speed downlink communications.

Keywords DICE - CubeSats · Ionospheric diagnostics · DC and AC electric fields · Plasma density · IDA4D · AIME · Constellation · Magnetometer · Langmuir probe

\title{
1 Introduction
}

The most significant advances in Earth, solar, and space physics over the next decades will originate from new, system-level observational techniques. The most promising technique to still be fully developed and implemented requires conducting multi-point or distributed constellation-based observations. This system-level observational approach is required to understand the "big picture" coupling between disparate regions such as the solar-wind, magnetosphere, ionosphere, upper atmosphere, land, and ocean. The national research council, NASA science mission directorate, and the larger heliophysics community have repeatedly identified the pressing need for multipoint scientific investigations to be implemented via satellite constellations (Heliophysics Roadmap 2009; Solar and Space Physics Decadal Survey 2012). The NASA Solar Terrestrial Probes Magnetospheric Multiscale (MMS) mission and NASA Earth Science Division's "A-train," consisting of the AQUA, CloudSat, CALIPSO, and AURA satellites, are examples of such constellations. However, the costs to date of these and other similar proposed constellations have been prohibitive given the "large satellite" architectures or the multiple launch vehicles required for implementing the constellations.

Financially sustainable development and deployment of multi-spacecraft constellations can only be achieved through the use of small spacecraft that allow for multiple hostings per launch vehicle. The revolution in commercial mobile and other battery powered consumer technology has helped enable researchers in recent years to build and fly very small yet

\author{
J. Petersen \\ L-3 Communications, 640 N 2200 W, Salt Lake City, UT, USA \\ S. Schaire \\ NASA Wallops Flight Facility, Wallops Island, VA, USA
}

C.R. Davis

LJT and Associates, 9881 Broken Land Parkway, Suite 400, Columbia, MD, USA

M. Bokaie

TiNi Aerospace, 2505 Kerner Blvd., San Rafael, CA, USA

M. Cousins

SRI International, 333 Ravenswood Ave., Menlo Park, CA, USA 
capable satellites, principally CubeSats. The CubeSat standard outlines a base "1U" nanosatellite which has a volume of approximately one liter (10 cm cube) and a mass $\leq 1.33 \mathrm{~kg}$, with scaling to larger CubeSats (i.e., 1.5U, 2U, 3U, 6U, etc.) then defined in terms of the baseline 1U (CubeSat Design Specification 2010). Most CubeSat projects thus far have used modern high-performance commercial electronics components, rather than the more expensive and less capable space grade components, and targeted low earth orbit insertions with relatively short $(<1$ year) mission lifetimes. A majority of the CubeSat activity and development to date has come from international academia and the amateur radio satellite community, but several of the typical large-satellite vendors have developed CubeSats as well. In 2004, CubeSats could each be fabricated and launched for as low as an estimated $\$ 65,000-\$ 80,000$ (David 2004). Recent government-sponsored CubeSat initiatives, such as the NRO Colony, NSF CubeSat Space Weather, NASA Office of Chief Technologist Edison and CubeSat Launch Initiative (CSLI) Educational Launch of Nanosatellites Educational Launch of Nano-satellites (ELaNa), the Air Force Space Environmental NanoSat Experiment (SENSE), and the ESA QB50 programs have spurred the development of very proficient miniature space sensors and technologies that enable technology demonstration, space and earth science research, and operational CubeSat based missions.

Sponsored by NSF CubeSat Space Weather and NASA ELaNa programs, the dualspacecraft Dynamic Ionosphere CubeSat Experiment (DICE) CubeSat mission exemplifies a next-generation advanced space research undertaking. DICE consists of a pair of $1.5 \mathrm{U}$ CubeSats launched into an eccentric low Earth orbit (LEO), with each satellite equipped with instrumentation to measure ionospheric in-situ plasma densities, DC and AC electric fields, and DC and AC magnetic fields. The space environment instrumentation on each spacecraft is tightly integrated with the CubeSat bus and structure, effectively creating a "sensor-sat" capable of comprehensive ionospheric diagnostics. The DICE spacecraft are orbiting at slightly differing velocities in nearly identical LEOs, permitting the de-convolution of spatial and temporal ambiguities of ionospheric observations. The DICE mission is being enabled by a number of groundbreaking CubeSat technologies, including miniaturized mechanism actuators and software definable communications system operating at $\geq 3 \mathrm{Mbit} / \mathrm{s}$.

In addition to having its own specific mission research objectives, DICE serves as a pathfinder for low-cost, multi-point CubeSat constellation observations of the Sun-to-Earth system. Future expectations are for multiple copies of DICE-like spacecraft to be inserted into orbit to enable networked measurements of the interactions between the magnetosphere and ionosphere due to varying conditions originating on the sun. This will help provide a comprehensive understanding of the Earth's global response to changing levels of solar activity. This constellation could contain up to 50-100 DICE CubeSats, all launched and inserted from a single vehicle, and able to acquire and transmit to ground nearly a Terabit of measurement data per day. This type of CubeSat-based constellation can be readily accommodated in the budget and programmatic constraints of a NASA Explorers program.

The balance of this paper presents the pioneering DICE mission design, development, implementation, and on-orbit performance and reviews the challenges faced in implementing a high-return science mission with limited resources. This paper is organized as follows: Sect. 2 discusses the DICE mission objectives. Section 3 presents mission design and implementation. Section 4 reviews the ground environmental and functional testing and calibration process of the spacecraft and sensors. Section 5 presents on-orbit performance and Sect. 6 provides conclusions and a summary. 


\section{Mission Objectives}

\subsection{Science Research}

DICE is primarily an exploratory Space Weather mission. Space weather refers to conditions in space (the Sun, solar wind, magnetosphere, ionosphere, or thermosphere) that can influence the performance and reliability of space-borne and ground-based technological systems. Ionospheric variability has a particularly dramatic effect on radio frequency (RF) systems; for example, large gradients in ionospheric electron density can impact communications, surveillance and navigation systems (Skone et al. 2004; Sojka et al. 2004). Some of the largest gradients are found on the edges of geomagnetic Storm Enhanced Density (SED) features which regularly occur over the USA in the afternoon during magnetic disturbances. The SED feature was first identified by Foster (Foster 1993) using the Millstone Hill incoherent scatter radar (ISR), although Total Electron Content (TEC) enhancements caused by SEDs had been observed much earlier (Klobuchar et al. 1968; Mendillo 2006). More recently, 2-D TEC maps obtained from global ground GPS receivers have shed new light on the space-time properties of mid latitude SED, and their relationship to plasmaspheric processes (Coster et al. 2003; Foster and Rideout 2005).

The formation and evolution of SED can be described by two related structures (see Fig. 1). The first is the formation of a greatly enhanced SED "bulge" of plasma which seems to preferentially originate at lower latitudes over the north American continent (between approximately $0-35^{\circ}$ latitude and -80 to $-130^{\circ}$ longitude) and appears correlated with storm-time prompt penetration electric fields (PPE) at low latitude (Foster and Coster 2007; Mannuci et al. 2007). The second is the formation and evolution of a narrow SED "plasma plume" that first forms at the base of the SED bulge, and then extends pole-ward into and across the polar cap. The SED plume appears to be strongly correlated with the expansion of the polar convection cells, and is thought by some investigators to be due to the existence of a strong sub-auroral polarization stream (SAPS) in the local afternoon/evening mid-latitude sector (Foster et al. 2004).

Several important research questions regarding SEDs were unanswered and were the motivation for the DICE mission. First, how exactly is the greatly enhanced plasma formed over the lower North American continent sector (the SED bulge) and what is the source of the plasma? Second, exactly what physical drivers are involved in the formation and evolution of the SED plume, and what is their relative importance? Finally, the precise relationship between the occurrence of penetration electric fields (PPEs), the subsequent expansion of the Appleton anomaly crests, and the development of SED is an open research question, particularly in terms of why there is an apparent preference for the USA geographic sector, shown in Fig. 1. Ultimately the large redistributions of ionospheric plasma interfere with radio communications and the SED plume causes GPS navigation blackouts for users over North America. Since modern society has come to rely upon radio and more increasingly GPS, the ability to understand and predict space weather effects on these services is of great importance.

To address the outstanding questions of SED science, the DICE mission focused on three main scientific objectives:

1. Investigate the physical processes responsible for formation of the SED bulge in the noon to post-noon sector during magnetic storms,

2. Investigate the physical processes responsible for the formation of the SED plume at the base of the SED bulge and the transport of the high density SED plume across the magnetic pole, and

3. Investigate the relationship between PPE and the formation and evolution of SED. 
Fig. 1 Horizontal distribution (latitude vs. local time) of F-region peak electron density (NmF2) from IDA4D for:

(a) October 30, 2003;

(b) November 20, 2003, units are $1 \times 1011 \mathrm{~m}^{-3}$ and DMSP ion drift vectors are shown

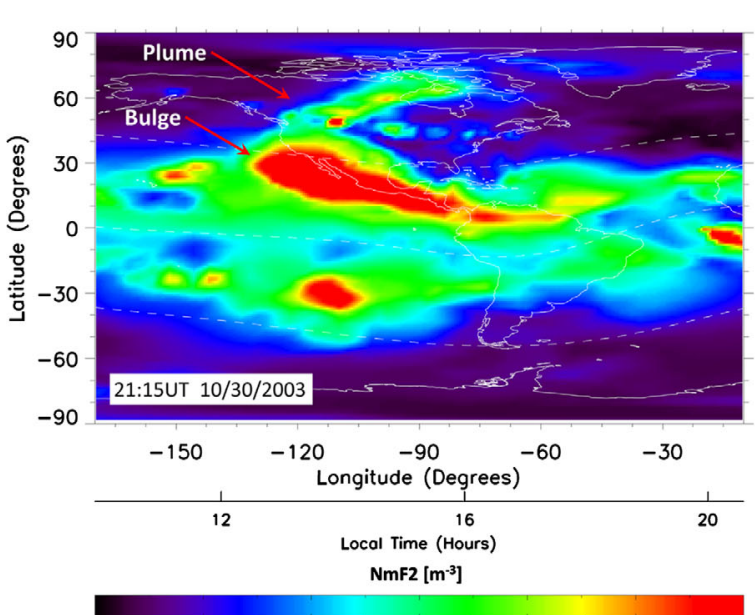

$0.00 e+008.33 e+11 \quad 1.67 e+12 \quad 2.50 e+12 \quad 3.33 e+12 \quad 4.17 e+12 \quad 5.00 e+12$

(a)

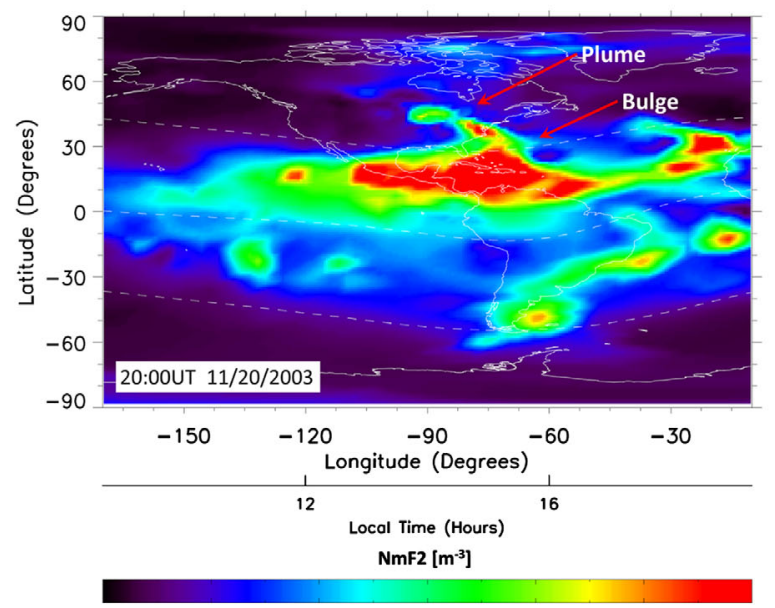

$0.00 e+008.33 e+11 \quad 1.67 e+12 \quad 2.50 e+12 \quad 3.33 e+12 \quad 4.17 e+12 \quad 5.00 e+12$

(b)

Progress on these science objectives can be achieved via in-situ ionospheric electric field and plasma density measurements from a multi-spacecraft mission. The electric field and plasma density measurements allow for the characterization of both the ionospheric plasma density and the electric field distribution. Ideally, what is needed to study SEDs is a set of simultaneous co-located plasma density and electric field measurements passing through the SED bulge and plume in the afternoon sector as they start to develop and then as they evolve. The goal was to place the DICE satellites in the afternoon sector between $\sim 13-17$ local time, where they would observe important features that have never been seen. With two spacecraft, it is possible to begin to separate temporal and spatial evolution of the SEDs. Thus, the two DICE spacecraft would begin to drift apart along track over the life of the mission so that they have to possibility of observing the same volume of plasma at slightly different times (time evolution). 
SEDs are large-scale features and the electric fields causing the SED bulge and plume are thought to have similar scales as the density plume. With an approximate spacecraft velocity of $7 \mathrm{~km} / \mathrm{s}$, a spacecraft will take approximately 10 seconds to traverse the SED plume; therefore a measurement cadence of at least 0.5 seconds was required of DICE to define the plume and the related (broader) plasma electric field structures. Ionospheric irregularities are of great interest for space weather and it has been shown that small scale irregularities form on the edges of large SED gradients (Foster 1993; Basu et al. 2003). The physical instability mechanism is not known, so directly measuring small scale electric fields in association with larger scale SEDs is quite valuable. For instance, observations at the Millstone Hill ISR have shown small scale electric field variability associated with the larger scale subauroral polarization stream (SAPS) channel (Foster et al. 2004), and it is possible that there is a physical connection between small scale AC electric field variability and the larger scale dynamics of the SED plume development right on the edge of the large scale gradient field.

\subsection{Technology Demonstration}

Communications is perhaps the most pressing technology development required to promote the use of CubeSats by government and commercial institutions for critical space missions. Global constellation-based science observations of the Earth system will require the capability to regularly downlink large amounts (e.g., >100 MBytes/day) of data to the ground. The greatest difficulty in fielding new communication technologies for CubeSats is surprisingly not the actual equipment development process but rather in working within the regulatory framework managed by the United Nations through the International Telecommunications Union (ITU) and the various national regulatory bodies. In the United States these are the National Telecommunications and Information Administration (NTIA) for government or the Federal Communications Commission (FCC) for civil and commercial application.

The relevant frequency bands in which government non-experimental, space-to-earth communications for space science applications are allowed are UHF (460-470 MHz, $<10 \mathrm{MHz}$ allocations) and S-Band (2200-2290 MHz, $<2 \mathrm{MHz}$ allocations). The S-Band has typically been used for Earth and space science missions. It has become heavily congested and it now would be politically difficult to obtain licenses for a large constellation of CubeSats operating in this band. The UHF band is essentially unused for space-to-earth communications because it is allocated for terrestrial, narrow-band fixed-to-mobile handset communications for entities like local police or fire services as the primary users. Interference at the satellite ground stations by primary users must be accepted which has limited its use for high profile government satellite programs. Although requiring additional effort initially, the DICE team wanted to make use of radio bands approved by the NTIA, as the resultant radio products would then be in regularly licensed government bands that could be rapidly infused into the space-user community. Therefore, DICE's major technology demonstration objective is to validate a reliable CubeSat high-speed communication downlink within the existing UHF allocated government approved radio band. Based on the CubeSat community space-to-ground communication technology existent at the time of the DICE mission inception in 2009, it was determined that a telemetry downlink capability $>1 \mathrm{Mbit} / \mathrm{s}$ would be a substantial leap forward in technology and enable significant observational data to be telemetered to the ground on a daily basis.

\subsection{Broader Impact}

As an NSF and NASA Educational Launch of Nanosatellites (ELaNa) sponsored CubeSat program, a key educational objective is that the DICE program is implemented and executed by university undergraduate and graduate students from a broad range of science and 
engineering disciplines, under mentorship and supervision of professional staff and faculty. This objective is motivated by the desire to engage, inspire, and provide training for the next generation of space engineers and scientists.

Additionally, the DICE team felt strongly that future low-cost, reliable research and operational CubeSat-based missions would most readily become viable if strong technical collaboration, based on common goals, was developed and fostered between small business, academia, government, and industry. The DICE team identified two areas which they felt were strategic to long term CubeSat mission success as well as being suitable for multiinstitution partnering:

1. Development of high-speed downlink communications for use on regularly licensed bands. This would include not only the design and implementation of a high-speed space to ground radio, but also the tools, facilities, and infrastructure to provide the ground tracking, communication link closure (i.e., high-gain ground antenna to enable the highspeed downlink), and data acquisition and management on the ground.

2. Development of miniature, reliable CubeSat deployment mechanism actuators. This refers to the need for proven actuators technologies, such as wax or shape-memory alloy, that can operate within a CubeSat power budget and occupy a very small percentage of a CubeSat volume.

\section{Mission Design and Implementation}

\subsection{Team Leadership and Organization}

The DICE Principal Investigator is Geoff Crowley, from ASTRA Inc. Charles Swenson, Utah State University/Space Dynamics Lab (USU/SDL), is the Deputy Principle Investigator. Chad Fish, USU/SDL, is the Program Manager and a science Co-Investigator. Science team Co-Investigators include Marcin Pilinski, Rick Wilder, and Irfan Azeem from ASTRA and Aroh Barjatya from Embry Riddle Aeronautical University. The project lead engineer is Tim Neilsen, USU/SDL. ASTRA Inc. is leading mission science analysis, with support from USU/SDL and Embry Riddle. The DICE engineering component was implemented at USU/SDL, with support from ASTRA Inc. and Embry Riddle. Major engineering industry partners include L-3 Communications, TiNi Aerospace, Clyde Space, ATK Aerospace, and Pumpkin Inc. GPS simulator testing occurred at the NASA Goddard Space Flight Center. Mission operations are conducted by USU/SDL, in collaboration with NASA Wallops Flight Facility and SRI International.

\subsection{Summary of Overarching Requirements}

The DICE mission design and implementation is the result of a number of drivers and requirements, including:

1. Provide a science and technology pathfinder demonstration for future CubeSat based multi-point measurement constellation missions as described in Sect. 1,

2. Meet the specific science, technical demonstration, and broader impact objectives as described in Sects. 2.1, 2.2, and 2.3 with particular emphasis on the science to mission functionality requirements,

3. Work within a very limited budget of $\sim \$ 1 \mathrm{M}$ and an aggressive development and delivery schedule of less than two years as was required under the NSF and NASA ELaNa grant and contract awards, and 


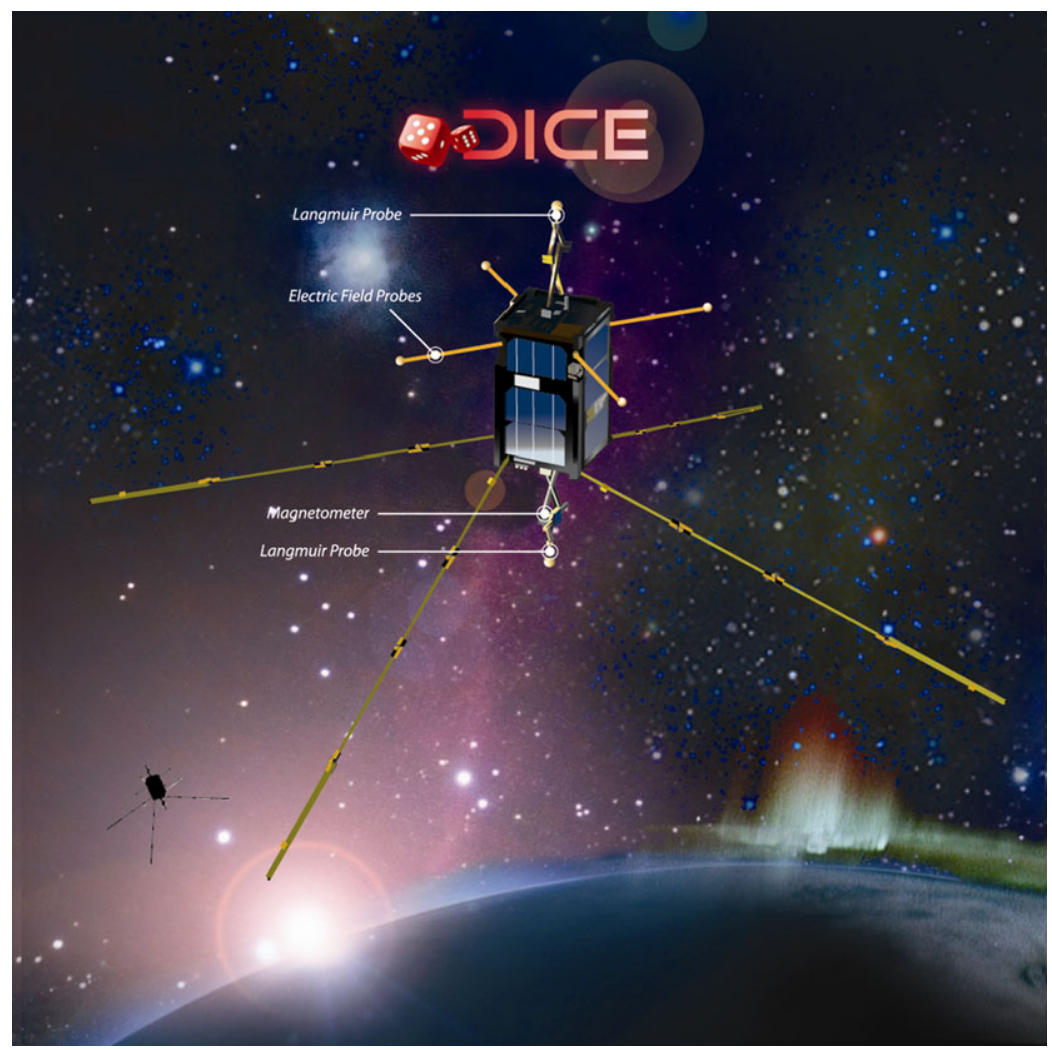

Fig. 2 The DICE mission is a next-generation NSF and NASA-sponsored space research mission formed around CubeSat technologies

4. Conform to the Poly-PicoSatellite Orbital Deployer (P-POD) containerized launch system for secondary payloads. The P-POD was developed and the design continues to be maintained by Cal Poly. It is a CubeSat deployment system that can house up to a $3 \mathrm{U}$ CubeSat. It provides a standardized system for integration with a launch vehicle. A significant portion of all CubeSats launched in the past decade have been carried to orbit in a Cal Poly P-POD. By conforming to this standard and expected similar follow-on standards, the DICE spacecraft design is a compatible forerunner for future opportunities.

Sections 3.3 to 5 describe the resultant DICE design details and implementation performance in response to these drivers and requirements.

\subsection{General Spacecraft Design-The "Sensor-Sat"}

The DICE mission is comprised of two 1.5U CubeSats, named Farkle (or DICE1) and Yahtzee (or DICE2). Each identical spacecraft carries two electric field probe pairs to measure in-situ DC and AC electric fields, two Langmuir probes to measure ionospheric in-situ plasma densities and temperature, and a complementary science grade magnetometer to measure in-situ DC and AC magnetic fields (see Figs. 2 and 3a).

The four electric field probe booms each extend $5 \mathrm{~m}$ from the spacecraft with electrically conductive spheres on the ends of the booms. The Langmuir probe spheres are supported on 
Fig. 3 (a) The DICE sensor-sat configuration (note: electric field booms shown in a partially deployed state); (b) Cut-away view of the spacecraft showing the tightly integrated components, sensors, and electronics

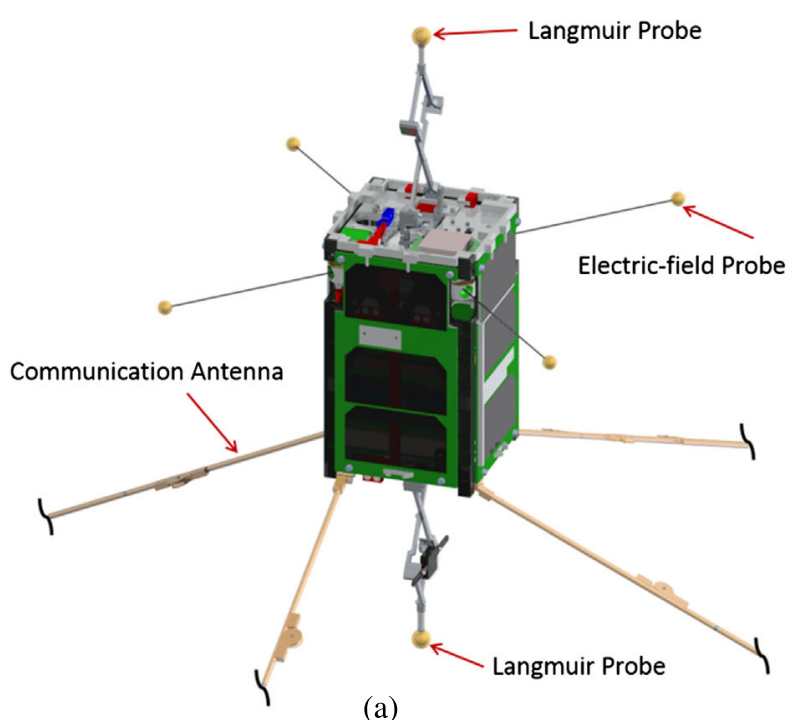

(a)

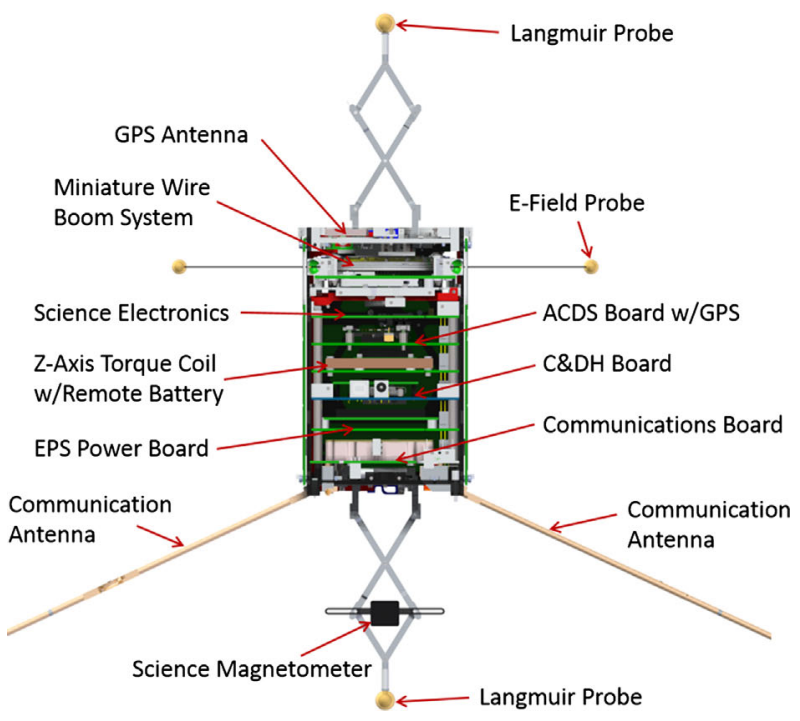

(b)

the top and bottom of the spacecraft by the use of scissor booms that extend $13 \mathrm{~cm}$ away from the spacecraft. The bottom Langmuir probe boom, as well as a cutout in the bottom spacecraft panel, supports placement of a science grade magnetometer. The four shorter booms on the bottom-side of the spacecraft comprise the UHF communications turnstile antenna and are $\sim 0.2 \mathrm{~m}$ in length. The UHF booms also increase the moment of inertia for the controlled spin of the spacecraft about the desired axis. Given the tight integration of these multiple sensors within the CubeSat platform, each of the DICE spacecraft is effectively a "sensor-sat" capable of comprehensive ionospheric diagnostics. 
Fig. 4 The DICE sensor map indicates the relative geometry between the ADCS and science measurement components, based on the spacecraft body coordinates

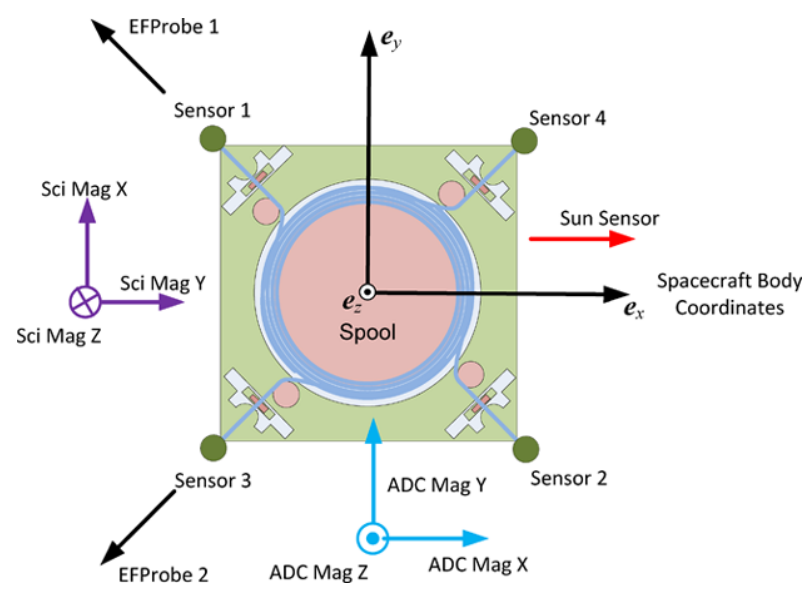

Each sensor-sat consists of a frame manufactured from a single block of 7075 Aluminum. An isogrid pattern was used to maintain structural integrity while reducing the mass of the structure. The top and bottom panels ( $+Z$ and $-Z$ faces) are also machined aluminum, with cutouts and mounting holes for release mechanisms, the Langmuir probes, GPS antenna, science magnetometer, and antenna mounts.

Electronics are mounted in a vertical stack (see Fig. 3b), and consist of, moving from the bottom of the stack up, (1) radio (L-3 CadetU) and antenna interface components, (2) electrical power system (EPS) that includes the primary battery, solar panel connections, and power system monitoring and conditioning electronics, (3) control and data handling (C\&DH) electronics, (4) Z-axis torque coil and secondary battery electronics, (5) attitude control and determination system (ADCS) electronics that include interfaces to a sun sensor, ADCS magnetometer, GPS receiver, and the spacecraft torque coils ( $Z$-axis in stack, $X$ - and $Y$-axis embedded in solar panel circuit boards), and (6) science instrument electronics that include interfaces to the electric field probes (EFPs), Langmuir probes (LPs), and science magnetometer (SciMag) sensors. The EFP boom deployment mechanism is mounted above the electronics stack, along with the main release mechanism for the antennas and scissor booms, and is discussed in detail in Sect. 3.3.1. The GPS antenna is located on the top panel. Solar panels with three solar cells each are attached on the long sides. A sun sensor views externally through a small cut out in one of the solar panels. An attitude determination magnetometer is located on the ADCS electronics board within the spacecraft. The use of magnetic materials in the design of the DICE spacecraft was restricted to minimize magnetic contamination of the SciMag measurements. The DICE sensor-sat instrumentation map, in spacecraft body coordinates, is shown in Fig. 4.

The DICE sensor-sats principally use passive thermal control with survival heaters for the batteries being the only active thermal control components. The solar panels are thermally isolated from the spacecraft body, effectively serving as thermal shields and reducing the internal temperature swing due to solar radiation. The radio dissipates a significant amount of power during its relatively short transmit duty cycle, and has a direct thermal conduction to the bottom exterior panel, which serves as the principal spacecraft radiator. This panel has a silverized FEP coated surface for optimum emissive properties. Temperature sensors are located throughout the sensor-sat.

The main release mechanism, which secures and releases the Langmuir probe scissor booms and UHF antennas, is comprised of a spring-loaded lever that runs from the top to the 
Fig. 5 The DICE instrument suite electronics are all integrated onto one miniature $\mathrm{PCB}$ $(\sim 9.5 \mathrm{~cm} \times 9.5 \mathrm{~cm} \times 1.5 \mathrm{~cm})$ that resides in the top of the DICE sensor-sat

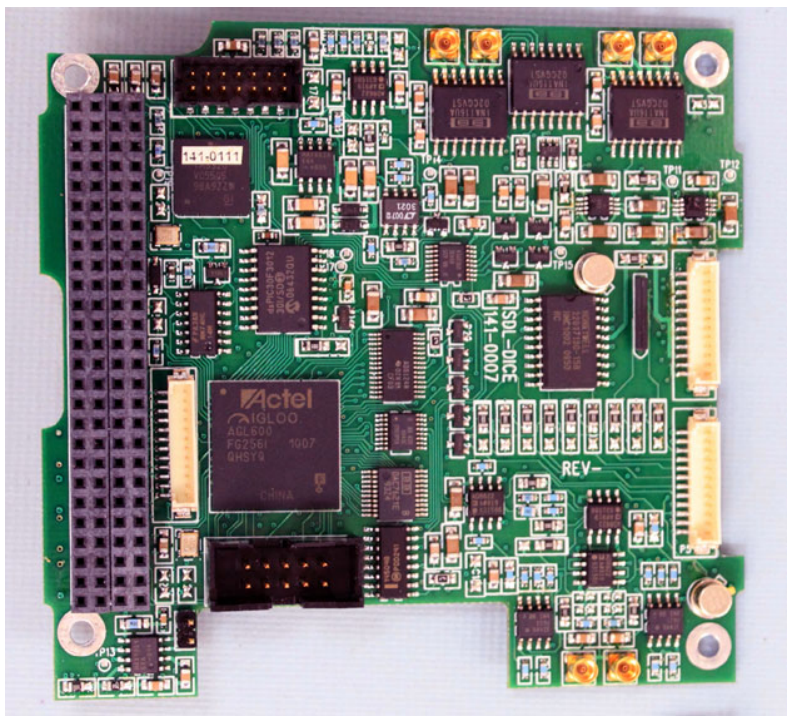

bottom of the CubeSat. It translates along the $-X$ direction to release both of the Langmuir probes, the antennas, and removes the first mechanical lock on the electric field spool. This mechanism is released by a shape-memory alloy Frangibolt locking actuator developed by TiNi Aerospace. The UHF antenna extensions, consisting of segments of brass tube and tungsten rod inserts, provide additional mass "away" from the spacecraft to enhance spin stability about the $Z$ axis (long axis) of the spacecraft.

\subsubsection{Science Instrument Suite}

The EFP, LP, and SciMag instruments electronics are housed in the spacecraft on a single printed circuit board (PCB), as shown in Fig. 5, weighing only 45 grams. The analog and digital sections of the board are physically isolated to minimize electro-magnetic interference (EMI) and RF coupling between signal measurement, data acquisition, and signal conditioning circuitry. When in full operation, the science PCB consumes $\sim 0.5 \mathrm{~W}$. An FPGA controls operation of the science instrument suite as well as data acquisition, digitization, time stamping, data formatting, and serial communications with the C\&DH sub-system such that packets of data passed to the C\&DH are merely stored in the telemetry buffer, ready for transmission by the radio. The DICE EFP, LP, and SciMag measurements are sampled simultaneously ( $<90 \mathrm{~ns}$ phase delay between each multi-instrument measurement event) at either a 35 or $70 \mathrm{~Hz}$ rate. The science instrument measurements are oversampled at a $17.9 \mathrm{kHz}$ rate and then co-added and decimated to improve signal to noise ratio (SNR) levels. The FPGA also controls the EFP boom deployment system.

Electric Field Probe (EFP) The DICE EFP is used to measure both DC and AC electric fields, and is an implementation of the double-probe class of in-situ electric field instruments that has been used for decades to observe electric fields in the space environment (Fahleson 1967). It operates by making measurements of the potential difference between two isolated, separate conductive sensors immersed in the plasma that are electrically isolated from the spacecraft electronics (see Fig. 3). Each EFP sensor metallic tip independently floats to a potential relative to the surrounding plasma. The EFP sensor tips are deployed $10 \mathrm{~m}$ apart, 
Fig. 6 A double probe electric field probe instrument with corresponding electric circuit model
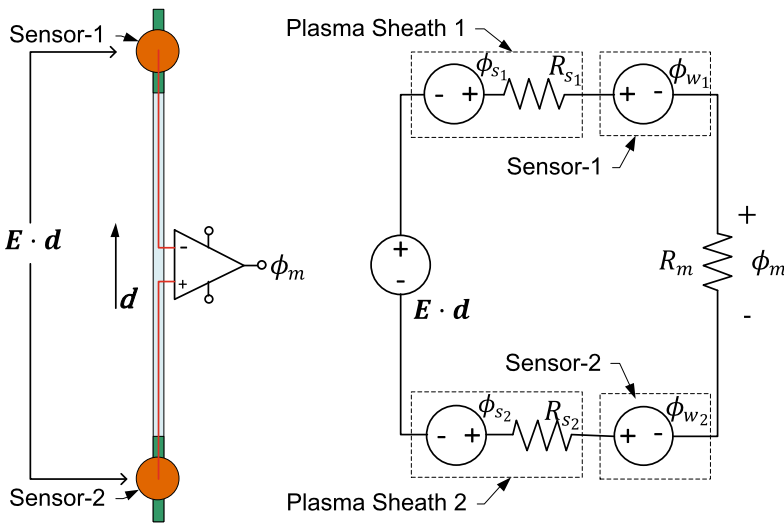

which is many ionospheric Debye lengths and are located in independent plasma sheath regions. The average electric field is derived from the measured differences in potential between the multiple EFP sensor tips, coupled with their separation distances. The potential difference is measured with a high impedance electrometer circuit. Four sensors and orthogonal separation baselines are used to observe the electric field in the plane, nominally perpendicular to the local magnetic field. The DC EFP, including sheath effects, surface work functions, and electronics input offsets, can be modeled using the circuit diagram shown in Fig. 6.

The ambient electric field dotted with the vector direction between the sensors, $\boldsymbol{E} \cdot \boldsymbol{d}$, is the driving signal. A linear model for the response of the plasma sheath to currents drawn from the plasma by the measurement electrons is a voltage source in series with a sheath resistance (Fahleson 1967). The work function of the probe surface for the emission and collection of electrons are shown as well as the input resistance, $R_{m}$, of the electrometer. The transfer function of the measurement voltage to the potential induced by the electric field is deduced from this lumped element model as

$$
\phi_{m}=\frac{\boldsymbol{E} \cdot \boldsymbol{d}+\left(\phi_{s 1}-\phi_{s 2}\right)+\left(\phi_{w 2}-\phi_{w 1}\right)}{1+\frac{R_{s 1}}{R_{m}}+\frac{R_{s 2}}{R_{m}}}
$$

which demonstrates that to operate close to an ideal measurement situation, a few criteria need to be met. First, $R_{m}$ must be greater than either $R_{s 1}$ or $R_{s 2}$ (the sheath resistances on each probe) by at least an order of magnitude to minimize the series resistive components due to the sheath. The DICE electrometer input impedance is $>10^{15} \Omega$. Second, the difference in potential due to the varying sensor sheath $\left(\phi_{s 1}-\phi_{s 2}\right)$ and work functions $\left(\phi_{w 2}-\phi_{w 1}\right)$ must be closely matched between sensors. Differences in sheath potentials is generally not a dominant issue as the floating potential of an object in space is dependent to first order upon electron temperature, photo emission/collection, and energetic particle induced emission/collection. The fact that two DICE EFP sensor tips are generally close to each other $(\sim 10 \mathrm{~m})$ relative to the large $(>10 \mathrm{~km})$ gradient scales for electron temperature and radiation means that these effects cannot cause long term changes in the double probe contact potential. The occasional "sun pulses" resulting from photoemission differences when one sensor is in sunlight and the other in spacecraft shadow are a feature due to changing sensor floating potentials. They have little impact on determining electric fields and actually provide an opportunity to observe electron temperature (Earle et al. 1989). Minimization of work function induced potentials is generally achieved by making sensor tips geometrically 
Fig. 7 The differential voltage signal from one double probe yields electric field information with spin period time resolution independent of the contact potential; spacecraft charging offsets the measurement range of the double probe electrometer circuit

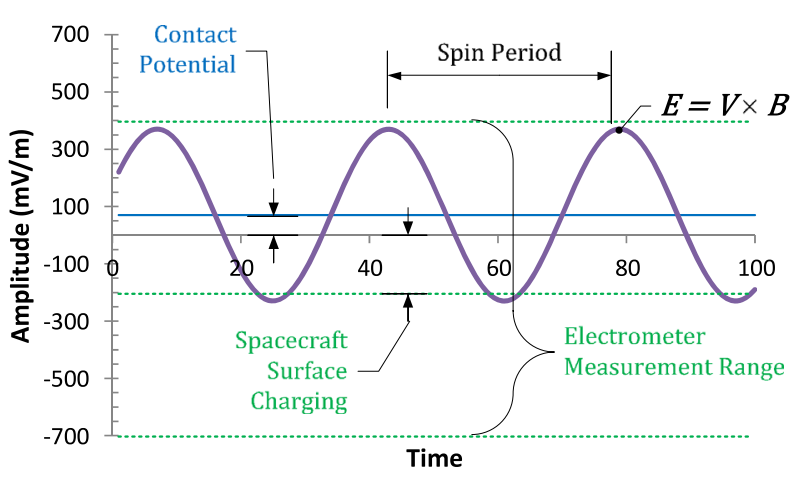

identical and spherical with surface properties that are stable in LEO. The DICE EFP sensor tips are identical gold-coated spheres. In practice, the combination of these sheath and work function terms is never zero and contributes a quasi-DC offset to the desired electric field measurement. This is often referred to as the contact potential. As an example, the Vector Electric Field Instrument (VEFI) double probe sensor on the Communication/Navigation Outage Forecasting System (C/NOFS) spacecraft has observed contact potential variation as great as $200 \mathrm{mV}$ over a one month period (private communications Robert Pfaff 2012). Reasons for the VEFI variations are not fully understood but are hypothesized to be a result of work function changes due to spacecraft contamination or reaction of the sensor surfaces with the thermospheric atomic Oxygen.

The major challenge in using double probes to measure quasi-DC fields in the space environment is the large induction field generated by the motion of the sensor in the Earth's magnetic field. The electric field in the moving frame is given by

$$
\boldsymbol{E}=\boldsymbol{V} \times \boldsymbol{B}+\boldsymbol{E}_{a}
$$

where $\boldsymbol{V}$ is the velocity of the satellite, $\boldsymbol{B}$ is the Earth's magnetic field and $\boldsymbol{E}_{a}$ is the ambient electric field of geophysical interest in the non-moving frame. The peak magnitude of the induction field for DICE in its 820 by $420 \mathrm{~km}$ orbit at $102^{\circ}$ inclination is $400 \mathrm{mV} / \mathrm{m}$. Removal of the induction field to better than $0.2 \%$ requires that the attitude knowledge of the double probe must be known to $\leq 0.1^{\circ}$ in an absolute sense, but additional information acquired from post-processing the direct $\boldsymbol{E}$ and $\boldsymbol{B}$ measurements helps to offset this stringent attitude knowledge requirement.

Figure 7 illustrates the elements of the double probe signal that are not due to $\boldsymbol{E}_{a}$. The spin of the double probe allows for the clear determination of contact potentials as a slowly changing DC offset and allows for the removal of the $\boldsymbol{V} \times \boldsymbol{B}$ component of the measurements without a precise attitude solution. The induction field has about a $4 \mathrm{mHz}$ bandwidth, down $50 \mathrm{~dB}$ from the peak, at the spacecraft spin frequency as $\boldsymbol{V}$ and $\boldsymbol{B}$ vary over an orbit. Thus the induction field can be removed by spectral filtering techniques with the consequence that some geophysical data will also be lost. The preferred removal approach is to estimate the spin, precession, and nutation rates of the double probe motion by modeling the measurement frame data using the torque free equations for rigid body motion. The $\boldsymbol{V} \times \boldsymbol{B}$ component can then be removed with the additional constraint that the long term orbital average of the zonal geophysical field is zero. This approach has similarities to drift meter data processing, and like the spectral technique, the removal can be done without a precise attitude solution. The orientation of $\boldsymbol{E}_{a}$ in the earth frame will then be known to the precision of the attitude solution. 
Fig. 8 A lumped element model of the unshielded wire booms of the DICE electric field probe

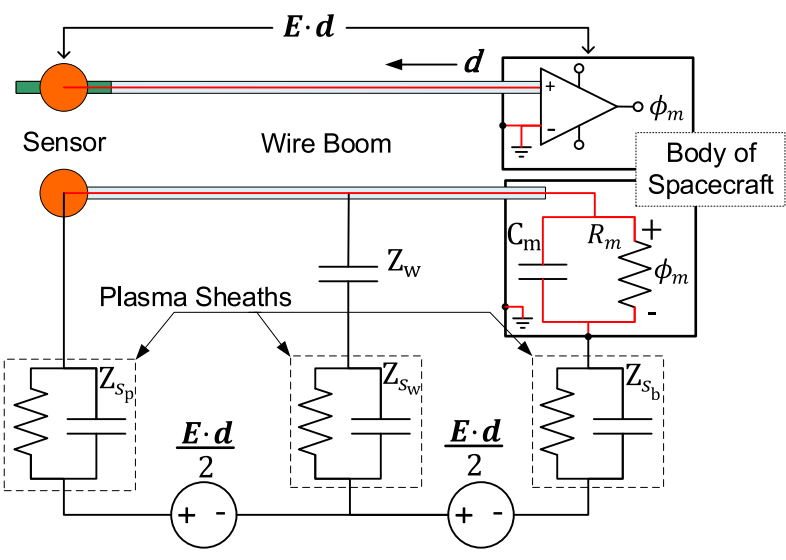

Spacecraft surface charging can shift the input range of the electrometer relative to the plasma environment, also illustrated in Fig. 7, and can cause clipping of the signal if pushed beyond the voltage supplies of the input electronics electrometer. Exposed potentials from the vehicle, such as Langmuir probes, solar cell interconnections, and spacecraft separation connectors can add a temporal variation to the vehicle surface charging. Depending on the common mode rejection ratio of the electrometer, a portion of these signals can appear in the EFP measurements. All of these error sources are controlled or eliminated on DICE by providing extended electrometer measurement ranges, measurement of the spacecraft floating potential, coating of solar cell interconnections, and by minimizing exposed connector area. Thus, the precision of a double probe for DC measurements is fundamentally set by variations of the contact potential of the probes to the plasma and the noise properties of the electrometer, but neither is a limiting factor on DICE to providing observations with 1 $\mathrm{mV} / \mathrm{m}$ precision. The measurement accuracy ultimately depends upon knowing the physical and electrical boom length and the disturbance effect of the spacecraft body on the ambient fields.

Precise knowledge of the electrical length of the DICE EFP is problematic due the unshielded wire used for the booms. The boom design originally included a shield composed of a thin conductive film on the wire surface but mechanical problems during integration test of the deploying mechanism resulted in its removal. Therefore the DICE EFP was in a similar electrical configuration, with the same data analysis issues as the San Marco instrument (Maynard 1998). A double probe with discrete tip sensors is preferred for making quasi-DC measurements because the electrical length is ideally the physical separation of the sensors. This property is lost without an electrical guard because electric field signals can be coupled into both the sensor and the boom wire via its capacitance to the surrounding plasma. Additionally, the $30 \mathrm{~cm}$ of unshielded boom wire within the body of the spacecraft adds a significant amount of shunt capacitance, $\sim 90 \mathrm{pF}$, limiting the high frequency response of the probe. A lumped parameter circuit model for one half of a double probe instrument with unshielded booms is presented in Fig. 8 .

The instrument is modeled as being composed of three elements: a spherical probe sensor, an insulated but unshielded boom, and the spacecraft body which hosts the electric field electrometer with a very high input resistance, $R_{m}$ and shunt capacitance $C_{m}$. The measured signal, $\phi_{m}$, is generated by a small current being driven through the probe by a potential between the probe and the spacecraft body due to the ambient electric field of strength $\boldsymbol{E} \cdot \boldsymbol{d}$. The complete circuit description includes the return current paths to and from the ambient 
Fig. 9 A transfer functions for the double probe on DICE using unshielded wire

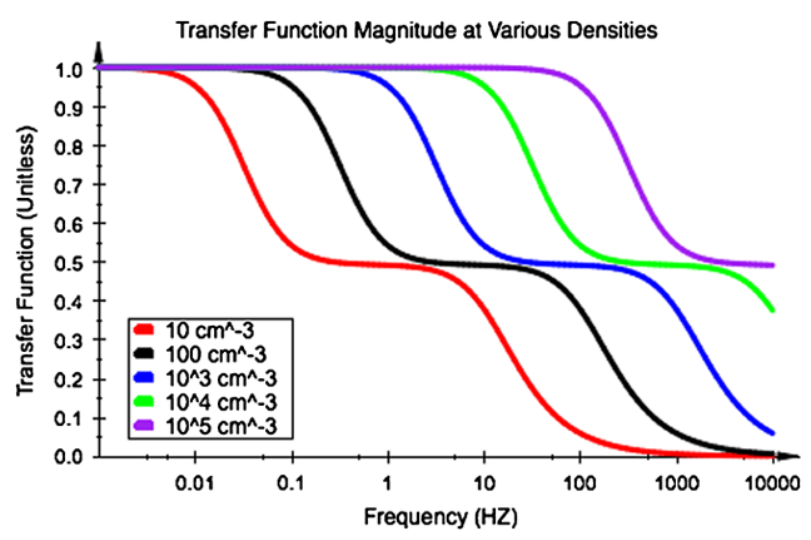

plasma through the various plasma sheaths. These are represented as lumped elements $Z_{s_{p}}$, $Z_{s_{w}}$ and $Z_{s_{b}}$ representing the parallel combination of a sheath resistance and capacitance. The sheath capacitance scales with the surface area $A$, of the element and the Debye length and the sheath resistance is approximated as

$$
R_{s}=\frac{\sqrt{2 \pi m_{i} k_{b} T}}{A n_{o} e^{2}}
$$

where $m_{i}$ and $T$ are the ion mass and temperatures in a stationary plasma of density $n_{o}$. The critical element in the unshielded model is the cylindrical capacitor which exists between the conductor of the wire boom and the plasma at its surface, $Z_{w}$. The separation between these conductors is equal to the thickness of the insulation on the wire with a distributed capacitance per fractional length given by

$$
\delta C_{w}=\frac{2 \pi \epsilon \epsilon_{r} \delta d}{\ln \left(\frac{b}{a}\right)}
$$

where $a$ is the diameter of the conductor and $b$ is the diameter of the conductor and insulation. The potential driving the distributed boom capacitance varies with position along the boom. An integration over the length of the boom gives an effective potential, $(\boldsymbol{E} \cdot \boldsymbol{d}) / 2$, that is applied to the total capacitance of the wire. This circuit can then be solved to determine a transfer function between the input potential and the output measurement as

$$
\frac{\phi_{m}}{\boldsymbol{E} \cdot \boldsymbol{d}}=\frac{Z_{m}\left(Z_{w}+\frac{1}{2} Z_{s_{p}}+Z_{s_{w}}\right)}{Z_{m}\left(Z_{w}+Z_{s_{p}}+Z_{s_{w}}\right)+Z_{s_{p}}\left(Z_{w}+Z_{s_{b}}+Z_{s_{w}}\right)+Z_{s_{b}}\left(Z_{w}+Z_{s_{w}}\right)}
$$

where $Z_{m}$ is the parallel combination of $R_{m}$ and the measurement shunt capacitance $C_{m}$. The magnitude of this transfer function is presented in Fig. 9 for the DICE double probe.

At low frequencies the probe behaves ideally with unity gain or as if the electrical length matches the physical length. At high frequencies the system behaves like a wire dipole antenna with gain, or electrical length, of 0.5 . The transition from signal being predominantly conducted by the tip sensor verses through the wire capacitance is problematic and is a function of the local plasma density. The second roll off is due to the measurement shunt capacitance and the resistance of the sheath around the wire boom. The calibration of the DICE sensor is complicated by the need to use the ambient density observed by the Langmuir probe and this transfer function to correctly calibrate the EFP data for plasma densities 


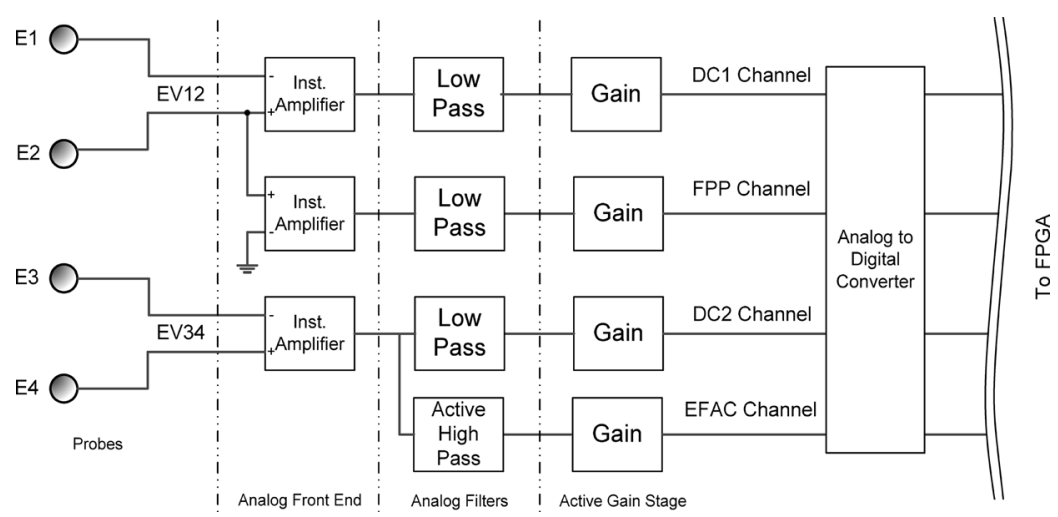

Fig. 10 The DICE EFP functional block diagram

less than about $1 \times 10^{4} \mathrm{~cm}^{3}$ given the 35 or $75 \mathrm{~Hz}$ sampling rates of the instrument. If the thin film shield had been included and actively driven then the DICE EFP would have been able to make measurements in much lower density plasmas without concerns over the effective length of the antenna or significant measurement shunt capacitance.

The DICE EFP measures the ambient ionospheric electric field via high input impedance electrometers, as illustrated in Fig. 10.

The instrument suite consists of four separate data streams deriving from four sensors deployed from the spacecraft body on wire booms. The data streams consist of two orthogonal DC channels, a floating potential channel, and an electric field spectrometer (AC electric field measurements) channel. The DC channels, EV12 and EV34, measure the electric field in the two dimensions perpendicular to the local magnetic field. The floating potential channel enables observation of changes of the spacecraft potential relative to the local environment, primarily due to LP sweeps, which then allows for cross-calibration of the LP plasma potential readings. The EFP spectrometer channel is used to observe higher frequency components of the field in four wave power spectral bands: 16 to $32 \mathrm{~Hz}, 32$ to $64 \mathrm{~Hz}, 64$ to $128 \mathrm{~Hz}$, and 128 to $512 \mathrm{~Hz}$. Each instrument has an analog front end which consists of a precision instrumentation amplifier. Filtering is then employed to set the desired frequency range for the measurements of each instrument and also to mitigate noise. Each of the channels has a gain stage to match to signals to the input range of the 16 bit ADC, where they are sampled and then communicated on to the C\&DH system via an FPGA for telemetry downlink. The EFP spectrometer implements the FFT, binning, and signal processing within the science PCB FPGA as well.

As the EFP AC and DC channels have varying input amplitudes across their frequency range, the channels are designed with different gains and filters to handle these frequency dependent variations. The signal amplitude inputs are driven by physical processes which change with ionospheric variability. Our team developed a model to determine the expected ionospheric electric field variability as a function of frequency. The information to generate and bound the electric field input model was taken from several sources: (1) on-orbit data from Dynamics Explorer 2 (DE2) and C/NOFS VEFI, (2) the predicted induced electric field from the spinning DICE sensor-sat, and (3) and predicted $1 / f$ noise, or pink noise. Our modeled and expected on-orbit DICE EFP DC and AC channel inputs levels are overlaid in Fig. 11. As shown in Fig. 11, The EFP DC channels measures large scale features in the ionospheres electric fields. 
Fig. 11 The DICE EFP makes measurements over a wide spectral and amplitude range

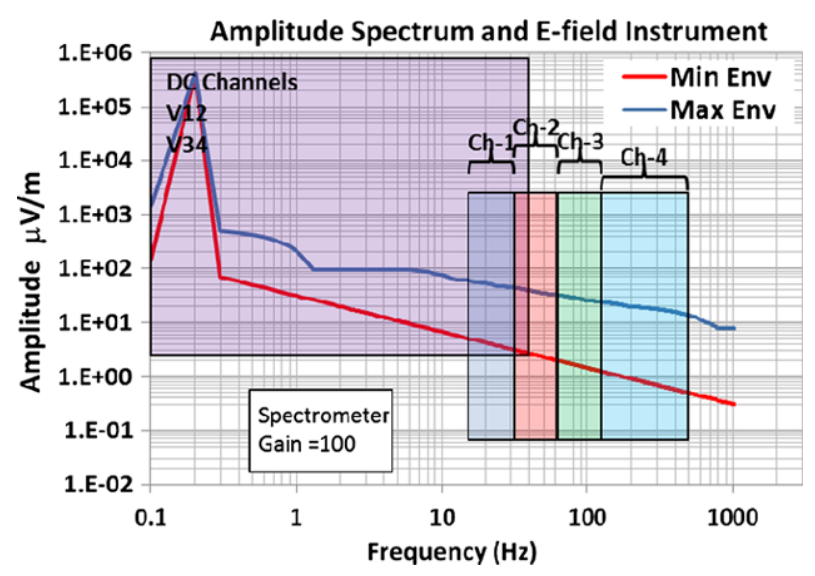

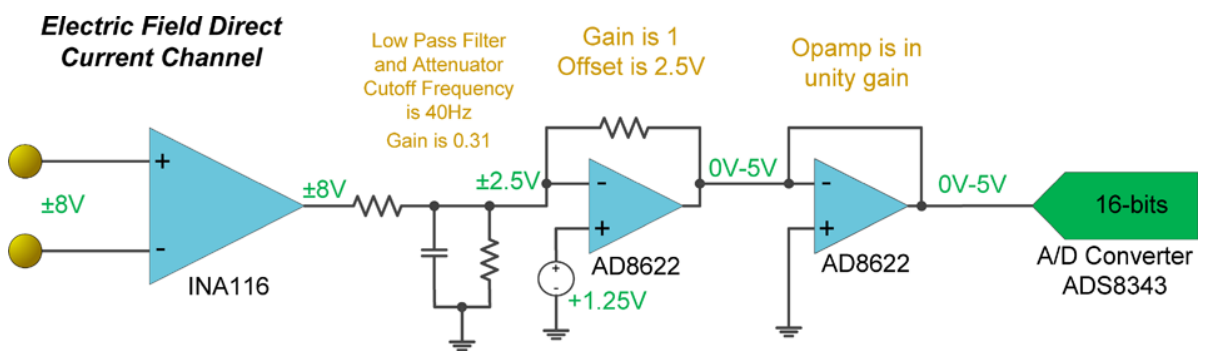

Fig. 12 The DICE EFP DC channel electronics schematic

A schematic of an EFP DC channel electrometer is shown in Fig. 12. Each EFP DC channel has a measurement voltage range of $\pm 8 \mathrm{VDC}$ and a resolution of $244 \mathrm{uVDC}$. The main preamplifier, a very high input impedance instrumentation amplifier, performs difference of the voltage seen across two sensor tips, and is then followed by two more amplifiers which provide a $40 \mathrm{~Hz}$ low pass filtering and matching function to the input range of the ADC. Finally, a gain buffer is used to cleanly drive the signal into the ADC. This same electronics configuration is used for the EFP floating potential channel, but the difference signal across the instrumentation amplifier is generated between one of the electric field sensor inputs and the sensor-sat chassis ground as opposed to being across two sensor inputs.

Ionospheric plasma can supply on the order of a few $\mathrm{pA}$ for an exposed $1 \mathrm{~cm}$ diameter sphere in LEO without changing its floating potential. To prevent "loading" of the ionospheric electric field signal by the EFP electrometer electronics, it is critical that the electrometer presents an effective $\geq 10^{15} \Omega$ input impedance to the space environment. This is accomplished through the use of high input impedance electronics and proper shielding and guarding techniques. On DICE, the EFP boom cables are composed of an inner conductor, which is the electrical tie between the electronics and sensor tip, and an outer Teflon insulator. Teflon was chosen given its high resistivity of $10^{18} \Omega \mathrm{cm}$, as well as its proven durability in the space environment. At the circuit board connection point, the EFP boom cables are then transitioned in and actively guarded from a connector header along the PCB track to the instrumentation amplifier inputs.

The EFP AC channel captures high frequency information $(16-512 \mathrm{~Hz})$ that is associated with waves or smaller scale features in the plasma and computes their spectral power. The 


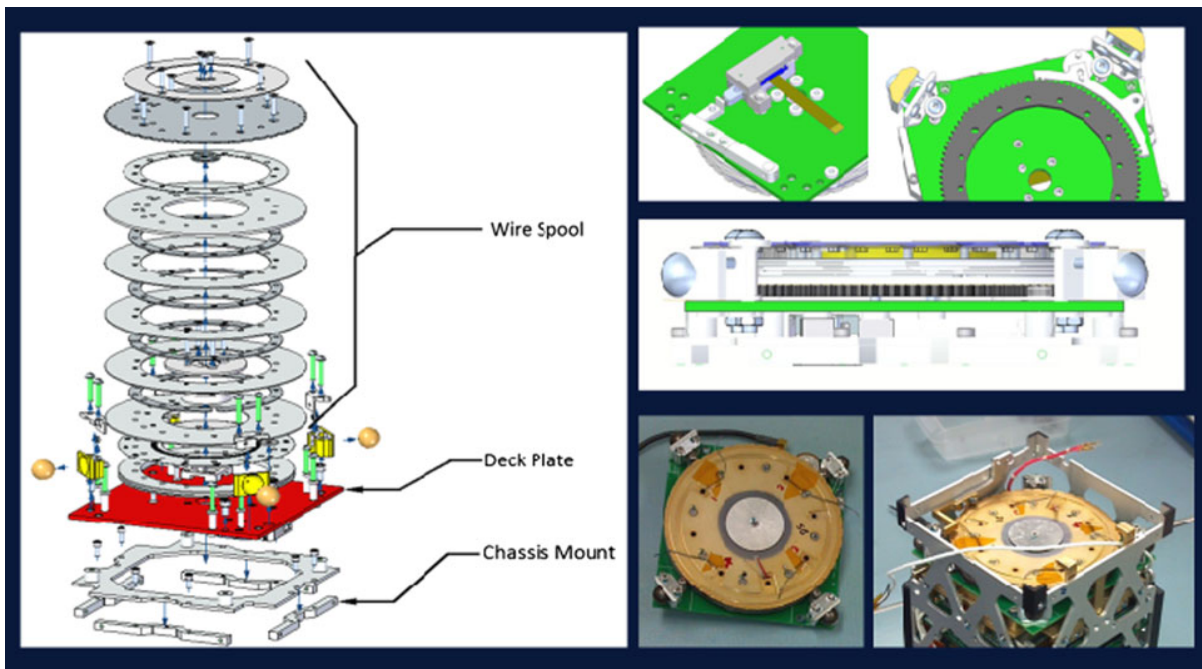

Fig. 13 The DICE electric field boom deployment mechanism includes a set of precision spools, specialized cabling and harnessing, and non-magnetic motor actuator to deploy $5 \mathrm{~m}$ booms on orbit from spinning nanoand pico-satellite platforms

EFP AC channel uses the same instrumentation amplifier and booms as the DC EV34 channel. Following the instrumentation amplifier, the signal is sent through a high pass filter and gain buffer and then into the ADC. The high pass filter is a 7-pole Butterworth, selected due to is sharp cut on and flat pass band amplitude response. The sampled signal is then sent to the FPGA where it is separated into different frequencies and summed up into four spectral bins.

The EFP boom deployment mechanism, shown in Fig. 13, provides the means to stow the lengthy $5 \mathrm{~m}$ electric field booms during launch and to control their slow deployment during on-orbit operations. Proper deployment of the booms on-orbit is a process that involves balance between centrifugal, centripetal, mechanical friction, motor stepping, and dampening forces acting on the booms while maintaining a stable deployment platform from the spacecraft. DICE is designed to rotate about the largest of its principal moments of inertia (major axis spinner) which is the lowest energy state possible for rotational motion and is therefore robustly stable. The response to an external torque is a shift of the instantaneous rotation vector resulting in the well-known coning or precession of the body axis in space about the angular momentum vector. This type of motion implies that the spacecraft has additional energy above the lowest energy state. Over time this energy will be dissipated, principally through slight flexures of the various booms on DICE, until the spacecraft returns to the minimum energy state with simple spinning motion about the major axis of inertia.

The deployment of the DICE EFP wire booms will be done gradually over time and in stages, such that the rotational motion of the spacecraft and wire booms are stable and oscillations are minimized. This will be guaranteed by keeping the spacecraft close to the minimum energy state throughout the deployment process. An incremental extension of the wire booms increases the principal moment of inertial of the spacecraft system and thus decreases the spin frequency due to conservation of angular momentum. The minimum energy state of the system also decreases with the larger inertia leaving an incremental amount of energy in the system as illustrated in Fig. 14. This energy will initially appear as forms of (1) heating of the deployment mechanism, (2) oscillation of the booms, or (3) coning 
Fig. 14 The DICE spacecraft spin rate will decrease as the wire booms are deployed; energy will be freed in the system as the booms are deployed
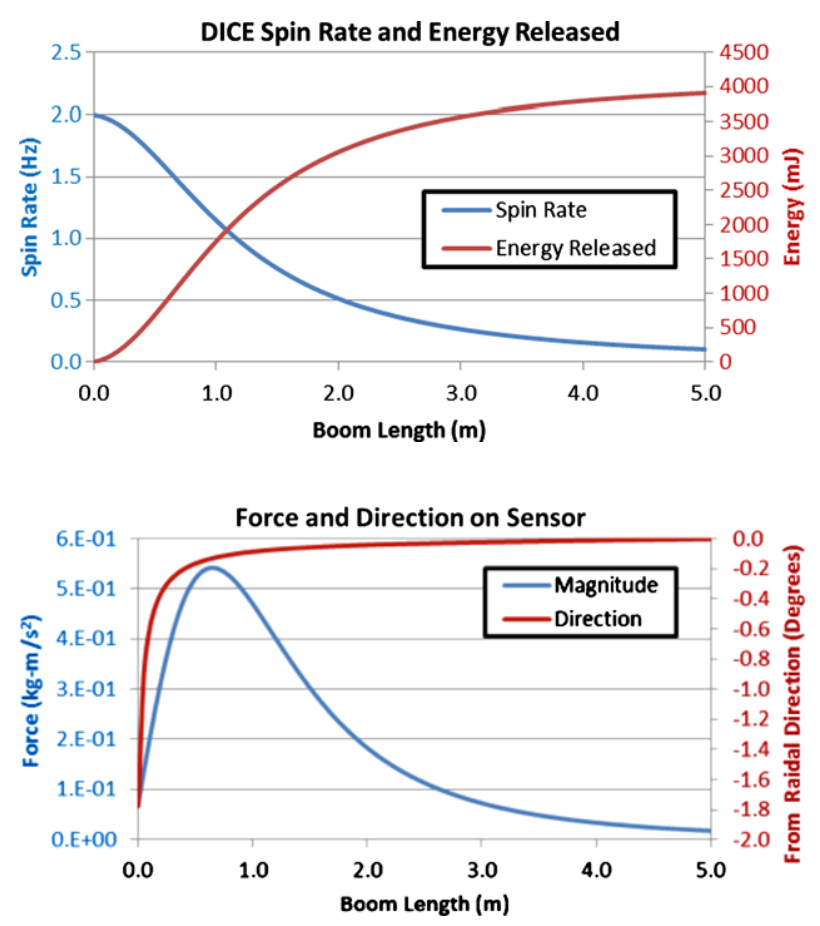

Fig. 15 The magnitude of the forces and the angle, from radial, of the force acting on the DICE wire boom sensors assuming a constant radial velocity of $1-\mathrm{cm} / \mathrm{s}$ and conservation of angular momentum

of the spacecraft body. Over time, energy dissipation effects will convert it all to heat and the spacecraft is left in a stable spin about the major axis of inertia. DICE will initially be spun up to $2 \mathrm{~Hz}$ and then the wire booms will slowly be deployed at a rate much slower than $1 \mathrm{~cm} / \mathrm{s}$ such that the internal energy dissipation mechanisms can dissipate the free energy. The peak tensile force within the wire booms increases initially as the wire booms are deployed and then drops as the booms reach their full extent as illustrated in Fig. 15. The direction of the force acting on the booms is essentially radial at slow deployment rates.

Langmuir Probe ( $L P)$ The LPs are used to primarily measure plasma density, $N_{e}$ and $N_{i}$, and temperature, $T_{e}$. They also provide measurements of the floating potential, $V_{f}$, and space potential, $V_{s}$. The LP measurements are based on the current-voltage $(I-V)$ response characteristics of a conductor immersed in a plasma at a Debye length or greater from surrounding structures (Barjatya et al. 2009). At $V_{s}$, the less massive electrons more easily migrate to the LP as no electric fields between the plasma and sensor surface exist. A sheath is formed around the LP sensor surface, and the resultant electric field is small and the surrounding plasma remains relatively undisturbed. Potentials applied to the LP sensor surface that are positive with respect to $V_{s}$ (electron saturation region) attract and accelerate electrons. Potentials applied negative with respect to $V_{s}$ (electron retarding region) begin to repel the less energetic electrons and to accelerate the ions. The $V_{f}$ is the point on the $I-V$ curve when both the electron and ion flows to the LP sensor surface are equal and therefore, the net current to the sensor surface is zero. Potentials applied negative relative to $V_{f}$ more strongly repel electrons, until a point is reached (ion saturation region) where only ions flow to the LP sensor surface, or electrons are emitted in the case of sunlit conditions. $T_{e}$ and $N_{e}$ can be derived from the values and slope of the electron retarding and saturation regions, respectively. $N_{i}$ is determined from the value of the ion saturation region. 
Fig. 16 The DICE LPs are deployed following launch, and extend from both ends of the sensor-sat so as to maximize wake free exposure throughout the orbit

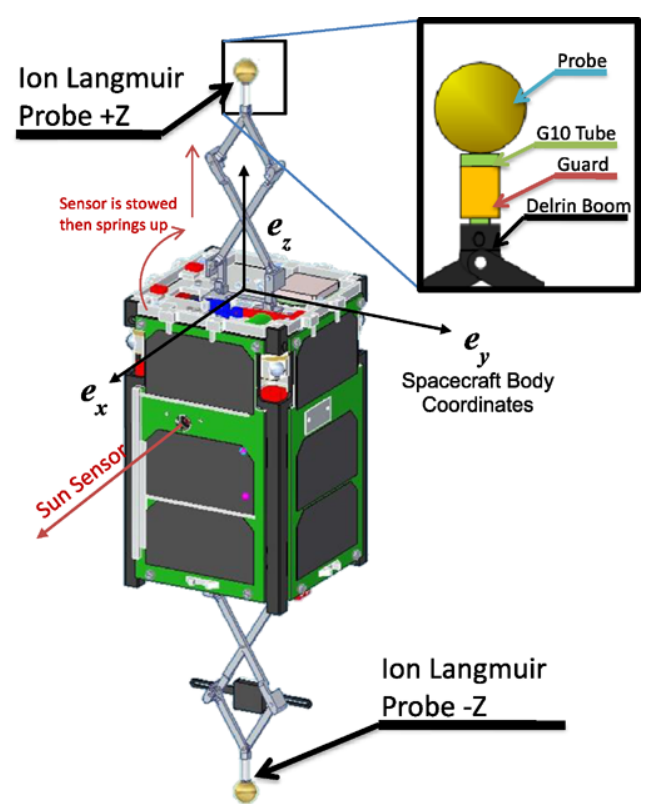

Traditionally, to acquire high resolution relative electron density measurement, an LP has been operated at a fixed-bias in the electron saturation region (Barjatya and Swenson 2006). However, at DICE spacecraft orbital speeds, the magnitude of ion ram current is significantly higher than the ion thermal current. Generally, with accurate knowledge of the spacecraft speed and the probe ram cross-section area, a LP operating in the ion saturation region can give high resolution absolute ion density measurements, minimize spacecraft charging, and are less susceptible to contamination effects (Steigies and Barjatya 2012). However, for certain conditions of electron temperature, this scenario may have significant errors, thus we will also periodically sweep the LP to determine when DICE is in high electron temperature regimes. DICE employs two separate spherical LP sensors operating in the ion saturation region that have an ion density measurement range of $1 \times 10^{10}$ to $2 \times 10^{13} \mathrm{p} / \mathrm{m}^{-3}$ and a minimum resolution of $1 \times 10^{9} \mathrm{p} / \mathrm{m}^{-3}$. They are also swept periodically in one direction from -4 to $+1.7 \mathrm{~V}$ DC to generate an $I-V$ curve, providing electron density and temperature and floating potential and spacecraft potential measurements. The EFP floating potential measurement is used to calibrate the LP potential point measurements and enable more accurate density and temperature measurements.

The two LP sensors are deployed by the use of scissor booms that extend $13 \mathrm{~cm}$ into the plasma environment from both the top and bottom of the DICE along its spin axis (see Fig. 14). The booms support $1.3 \mathrm{~cm}$ diameter end spheres. These spheres are gold-plated and conductive, but the booms themselves are electrically insulated from the plasma environment. Gold was chosen as the surface material because of its high stability in an atomic oxygen environment and because the radiative equilibrium temperature for a gold surface is significantly elevated and passively cleans the surfaces of LP sensors of contaminates. A clean gold surface presents a reasonably uniform work function (Camp et al. 1991; Amatucci et al. 1993). The booms also support conductive cylindrical guards located just below the sensor spheres, which are driven at the same potential as the spheres but maintained electrically isolated from the spheres. See Fig. 16 for the details of the LP sensor configuration. Along each spacecraft's orbital path, spin-stabilized attitude coupled with the LP 
Fig. 17 Example

magneto-resistive circuit, using a

simple Wheatstone bridge and

amplifier electronics set

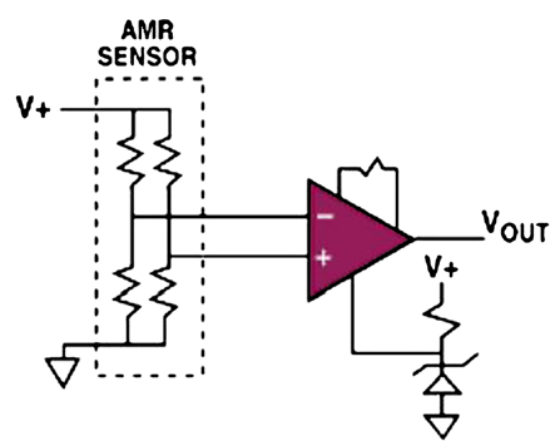

deployment geometry results in at least one of the two LP spheres always facing the velocity vector ram direction (see Fig. 53). The LP sensors are nominally biased to -4 VDC to repel electrons and allow for ion ram current measurements during flight. Due to the nature of the orbit and the variation of the ionosphere, the Debye length is expected to vary anywhere between a few $\mathrm{mm}$ to upwards of $15 \mathrm{~cm}$. However, due to a high spacecraft velocity that far exceeds the local ion mobility, the ion ram current collected by the LP sensors will not be affected by Debye lengths that are larger than the DICE spacecraft and LP sensor dimensions.

The DICE LP electronics are based around simple high input impedance, low noise transimpedance amplifier circuitry. Known voltages are applied to the spherical sensors and associated current flow to/from the ionosphere is measured. A $40 \mathrm{~Hz}$ low pass filter follows the current to voltage transducer, after which a gain amplifier is used to drive the ADC input. The oversampled LP data is co-added and decimated in the FPGA and transmitted to the C\&DH for telemetry transmission.

Science Magnetometer (SciMag) The DICE SciMag is used to measure both DC and AC magnetic fields, and is implemented using anisotropic magneto-resistive (AMR) sensing elements. AMRs are based on a nickel-iron (Permalloy) thin film deposited on a silicon wafer that is patterned as a resistive strip. The film's properties cause it to change resistance in the presence of a magnetic field. An external magnetic field applied normal to the side of the film causes the magnetization vector to rotate and change angle. This in turn will cause the resistance value to vary $(\Delta R / R)$ and produce a voltage output change in a resistive divider circuit such as a Wheatstone bridge. The reaction of the magneto-resistive effect is very quick and not limited by coil matching, with typical bandwidths on the order of a few MHz. The electrical interfacing to the AMR components is very straightforward. An example circuit is shown in Fig. 17.

The DICE SciMag is implemented using two-axis and single-axis AMR chip sets mounted on a small PCB that is then enclosed and intended to mount on either the $-Z$ axis LP boom or spacecraft deck plate, as shown in Fig. 18. The $-Z$ boom mount configuration is ideal in that it locates the SciMag further away from the spacecraft. However, the actual DICE flight build has the SciMag mounted to the $-Z$ deck due to some required fine tuning of the $-Z$ axis LP boom for proper accommodation of the SciMag PCB that was not able to be completed before launch. The SciMag has a measurement range of $1.5 \mathrm{mT}$ and a resolution of $5.5 \mathrm{nT}$ in each axis.

As AMR elements can become saturated in high fields (i.e., magnetic field domains line up incorrectly and oppose the field when exposed to high magnetic field), the SciMag has a built in degauss circuit that can be fired periodically to "refresh" the magnetometer readings 
Fig. 18 The DICE flight 3-axis SciMag is located on the $-Z$ axis of the sensor-sat

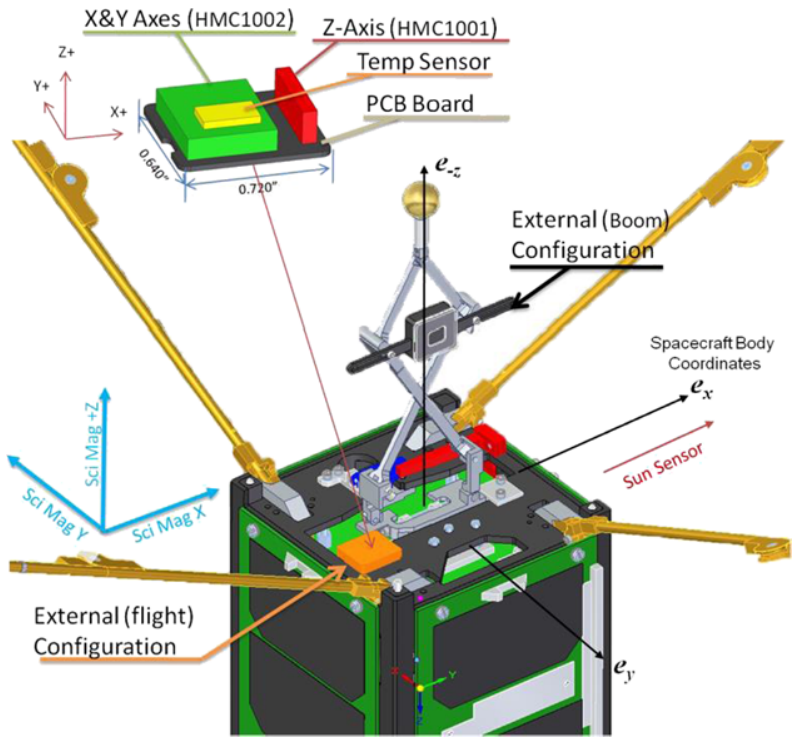

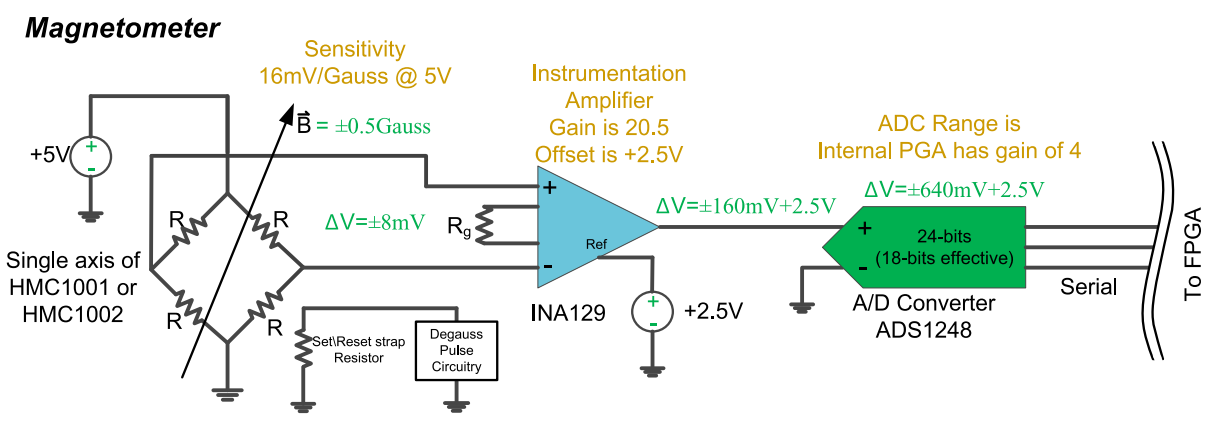

Fig. 19 The DICE SciMag channel electronics schematic

on orbit. Additionally, AMR element responses are sensitive to temperature, requiring a thorough temperature calibration of the SciMag.

The DICE SciMag circuit schematic is shown in Fig. 19. An instrumentation amplifier interfaces with the AMR implemented Wheatstone bridge. The difference signal is then sent directly into an ADC, which is a high resolution device capable of 24 bits of resolution, although only 18 bits of information are transmitted. This level of resolution is more than enough for the science requirements. The SciMag ADC also contains a digital filter that is set to $320 \mathrm{~Hz}$. This filter precedes the ADC sampling. The oversampled SciMag data is co-added in the FPGA and transmitted to the $\mathrm{C} \& \mathrm{DH}$ for telemetry transmission.

\subsubsection{Electrical Power Sub-system}

The DICE solar cell and battery power sub-system is a commercial Clyde Space LTD CubeSat electrical power system (EPS). It has a centralized architecture that is comprised of a monitor and control board, lithium-ion batteries, and custom solar panels that include embedded torque coil windings. The EPS provides an independent monitor of the overall power 
Fig. 20 Clyde Space EPS — centralized architecture used on the DICE CubeSat
Fig. 21 DICE EPS lithium polymer batteries provide significant energy density and long-life operation
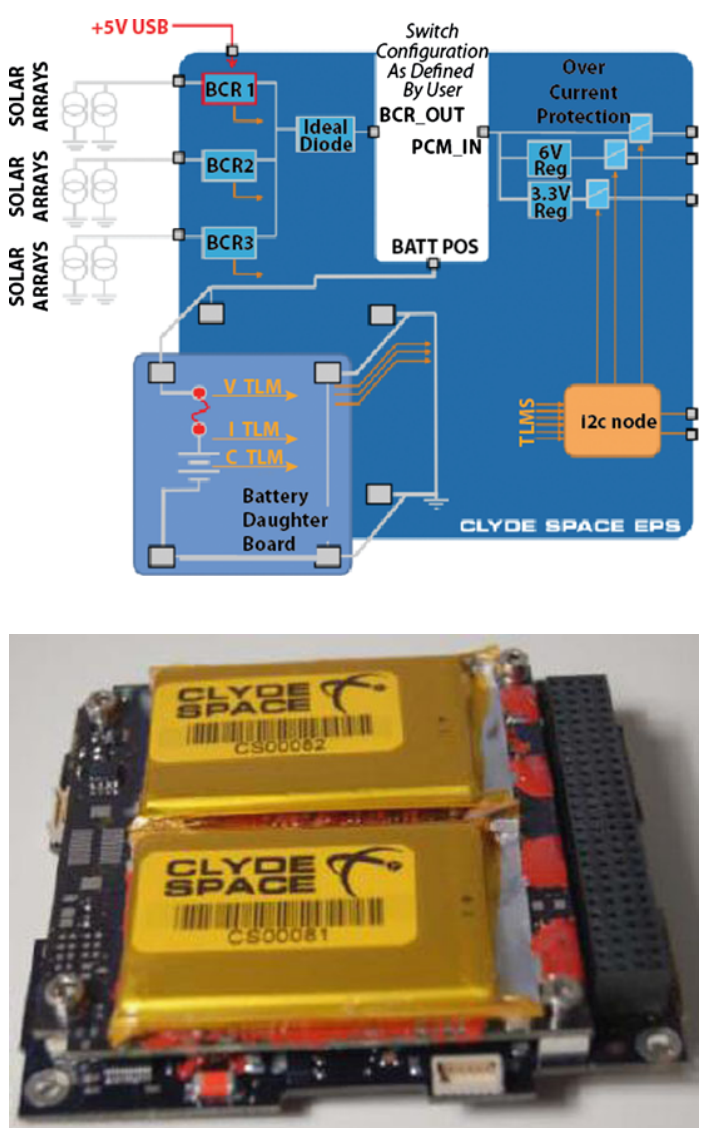

condition on the spacecraft and has the ability to recycle or switch off power to the remainder of the spacecraft upon detection of warnings, errors, or anomalies. The EPS maintains a nominal $8.2 \mathrm{~V}$ battery bus, and provides conditioned 5 and $3.3 \mathrm{~V}$ lines from the battery bus.

Figure 20 shows a block diagram of the DICE EPS. The design employs a peak power tracking algorithm to regulate the solar array. A dedicated Battery Charge Regulator (BCR) exists for each of three separate solar array inputs. The output of the BCRs pass through a series of switches designed to disconnect the battery, loads, and secondary regulators from the power source per requirements. Three power lines follow the switches; the first is the unregulated battery bus. The other two are regulated $5 \mathrm{~V}$ and $3.3 \mathrm{~V}$, respectively. The battery is a $2 \mathrm{SnP}$ lithium-polymer cell chemistry (see Fig. 21) where " $n$ " indicates the number of parallel strings and each string has two cells in series. This battery configuration equates to a maximum voltage of $8.4 \mathrm{~V}$ per string. The EPS is designed to charge the battery to a maximum of $8.3 \mathrm{~V}$ which allows for a longer life or more battery charge/discharge cycles. Once the battery is charged to the maximum voltage, the BCR maintains the voltage at that level. The sun regulated battery bus is output directly to the loads. Figure 22 shows the high efficiency EPS operation, as controlled by the BCRs. The DICE EPS operates above $80 \%$ for small to heavy loads.

The DICE power budget is shown in Table 1. The power budget loads are based on measurements acquired during ground testing. The power budget generations (i.e., solar panel outputs) are based on estimates. Orbit average power (OAP) is determined by the percent 
Fig. 22 DICE electrical power system and battery charge regulator efficiency

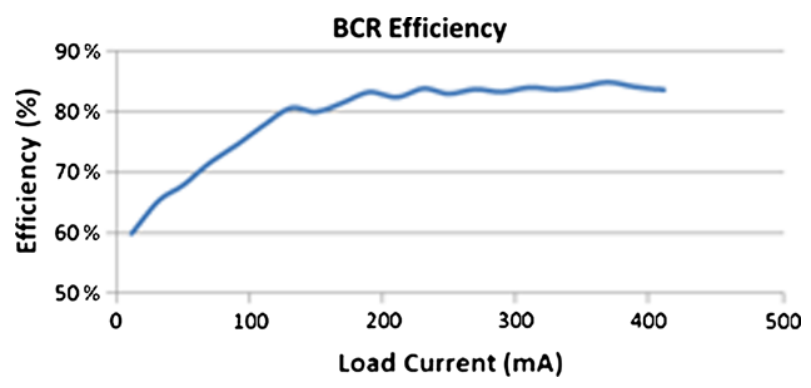

of time the load is on per orbit. Both margin and contingency are added into the budget to allow for errors in estimations. Approximately 1.8 W OAP are required to operate DICE in science mode, while the expected OAP generation is expected to be on approximately $2.7 \mathrm{~W}$.

\subsubsection{Command and Data Handling Sub-system}

The C\&DH, running a Pumpkin Inc. Salvo real-time operating system (RTOS) on a Pumpkin PIC24 processor card, is the central point by which the sensor-sat is controlled. The C\&DH sub-system ensures that the spacecraft is operating nominally, responds to warnings or errors, organizes acquisition of housekeeping (HK) data, and organizes acquisition of and compresses data from the science instruments. The C\&DH also manages transfer of all spacecraft data to the radio for storage and downlink upon command from the ground. A block diagram of the DICE sensor-sat functionality and interconnections is shown in Fig. 23. Both the radio and instruments can be reset from the $\mathrm{C} \& \mathrm{DH}$. The radio in turn monitors the C\&DH system and can reset it in case of lockups caused by radiation. Uplinked commands received by the radio are forwarded on to the C\&DH sub-system for execution. The primary means of keeping spacecraft event and measurement time are through a software based clock located in the C\&DH. This master clock synchronizes with on-orbit GPS time locks. A secondary, very precise crystal based clock is located on the science instrument electronics circuit board and time codes from this clock are embedded in the acquired science measurement stream. Spacecraft ephemeris data is also passed onto the C\&DH from the GPS receiver and included in the DICE downlink telemetry. These detailed C\&DH interactions are schematically shown in Fig. 24.

The flight software (FSW) on DICE is implemented via the Salvo RTOS for all flight software operations. Salvo is a simple, cooperative RTOS which provides event-driven, priority-based multitasking as wells as inter-task communications and synchronization. The FSW is comprised of a set of Salvo tasks, each with a distinct function, as shown in Fig. 25. All communication between tasks occur using Salvo messages and message queues. These messaging facilities involve using pointers to data structures in memory. Each pointer can be sent as either a simple "message" from one task to another or inserted into "message queue". At any given time, there can be multiple messages in a single message queue. In the FSW, each message queue will only be read from by a single task. Typically, each message queue will also be written to by a single task with a few exceptions (e.g. the System Messages Queue may be written to by any task).

The FSW implements three distinct sensor-sat modes: Safe, Standby, and Operational. The spacecraft mode affects which spacecraft functions and uplink commands are acceptable. The sensor-sats boot into Safe mode and only transition to Standby or Operational modes via ground-based commands. The allowed functions in each mode are shown in Table 2 . 
Table 1 DICE mass and power budget

\begin{tabular}{|c|c|c|c|c|}
\hline Subsystem & Mass (g) & Volume $\left(\mathrm{cm}^{3}\right)$ & $\mathrm{OAP}(\mathrm{mW})$ & $\mathrm{PP}(\mathrm{mW})$ \\
\hline \multicolumn{5}{|l|}{ Electronics Stack } \\
\hline Wire Boom Spool & 275 & $9.6 \times 9.6 \times 2.77$ & 17.0 & 169.4 \\
\hline Science Board & 45 & $9.6 \times 9.1 \times 1.1$ & 411.4 & 411.4 \\
\hline ADCS Board & 52 & $9.6 \times 9.1 \times 1.5$ & 226.3 & 226.3 \\
\hline GPS Receiver & 23 & $7.15 \times 4.6 \times 1.1$ & 61.8 & 1124.0 \\
\hline Battery Board & 125 & $8.2 \times 4.1 \times 1.45$ & - & - \\
\hline On Board Computer & 102 & $9.6 \times 9.1 \times 1.65$ & 78.7 & 78.7 \\
\hline Power Board & 163 & $9.6 \times 9.1 \times 1.65$ & 344.9 & 344.9 \\
\hline Cadet Modem & 73 & $6.9 \times 7.4 \times 1.35$ & 514.4 & 11439.6 \\
\hline Antenna Interface & 52 & $9.6 \times 9.6 \times 2.0$ & 30.0 & 30.0 \\
\hline Science Magnetometer & 25 & $1.83 \times 1.63 \times 0.7$ & 108.9 & 108.9 \\
\hline Sun Sensor & 50 & $3.3 \times 2.24 \times 9.15$ & 30.3 & 30.3 \\
\hline Subtotal & 985 & & 1823.6 & 13963.3 \\
\hline \multicolumn{5}{|l|}{ Solar Panels } \\
\hline (+) X-Solar Panel & 55 & $15.7 \times 8.3 \times 0.41$ & 682.5 (Generated $)$ & 3000.0 \\
\hline (-) $X$-Solar Panel & 58 & $15.7 \times 8.3 \times 0.41$ & $682.5($ Generated $)$ & 3000.0 \\
\hline (+) $Y$-Solar Panel & 58 & $15.7 \times 8.3 \times 0.41$ & $682.5($ Generated $)$ & 3000.0 \\
\hline (-) $Y$-Solar Panel & 58 & $15.7 \times 8.3 \times 0.41$ & $682.5($ Generated $)$ & 3000.0 \\
\hline Subtotal & 229 & & $2730.0($ Generated $)$ & 12000.0 \\
\hline \multicolumn{5}{|l|}{ Attitude Control } \\
\hline$X$-Torque Coil & 8 & $15.7 \times 8.3 \times 0.064$ & 1.5 & 151.3 \\
\hline$Y$-Torque Coil & 8 & $15.7 \times 8.3 \times 0.064$ & 1.5 & 151.3 \\
\hline$Z$-Torque Coil & 63 & $6.84 \times 8.52 \times 0.89$ & 1.5 & 151.3 \\
\hline Subtotal & 79 & & 4.5 & 453.8 \\
\hline \multicolumn{5}{|l|}{ Structure and Mechanisms } \\
\hline Thermal & 0 & & 20.0 & 20.0 \\
\hline Release Mechanism & 89 & $8.63 \times 9.55 \times 16.95$ & 0.0 & 8200.0 \\
\hline Scissor Boom (+) $Z$ & 42 & $5.0 \times 1.6 \times 15.22$ & - & - \\
\hline Scissor Boom (-) $Z$ & 42 & $5.0 \times 1.6 \times 15.22$ & - & - \\
\hline UHF Antenna & 92 & $1.9 \times 1.8 \times 21.35$ & - & - \\
\hline Inertia Balance & 272 & $36.7 \times 1.75 \times 0.43$ & - & - \\
\hline Wiring & 111 & - & - & - \\
\hline GPS Antenna & 28 & $4.5 \times 4.5 \times 0.77$ & - & - \\
\hline Structure & 310 & $17.025 \times 10 \times 10$ & - & - \\
\hline Subtotal & 986 & & 20.0 & 8220.0 \\
\hline Total & 2200 & & 1848.1 & 34637.0 \\
\hline
\end{tabular}

$\mathrm{OAP}=$ Orbit Average DC Power, $\mathrm{PP}=$ Peak Power 


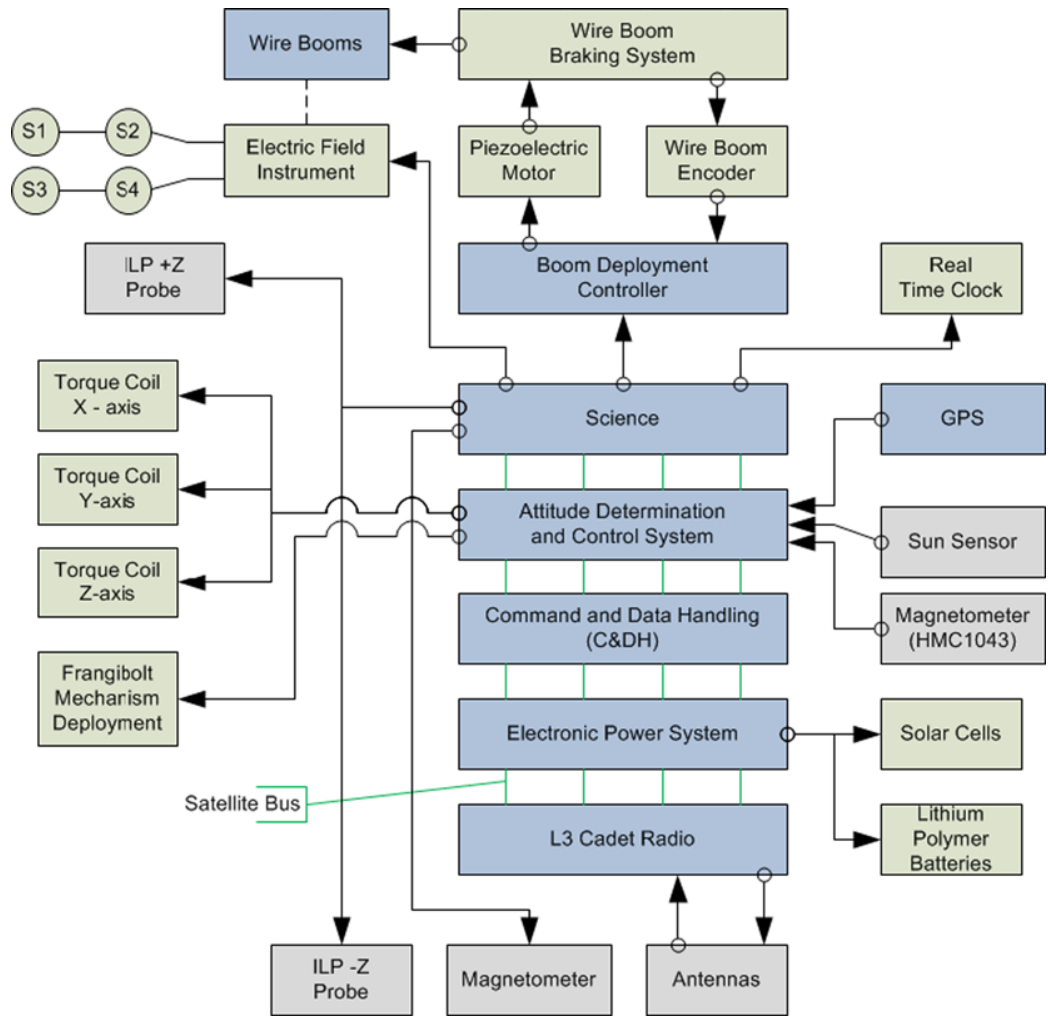

Fig. 23 The DICE sensor-sat is a tightly coupled set of sub-systems that are controlled and managed via the $\mathrm{C} \& \mathrm{DH}$

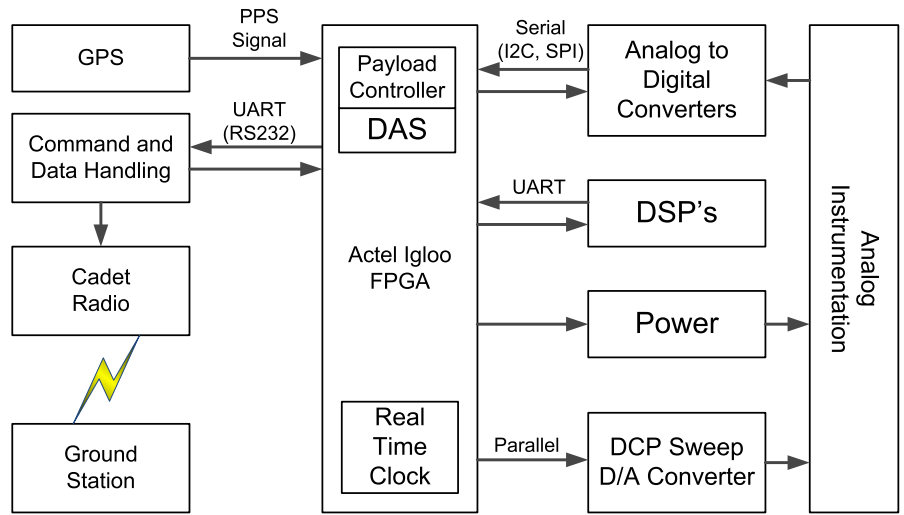

Fig. 24 The C\&DH directs data acquisition and formatting throughout the sensor-sat 


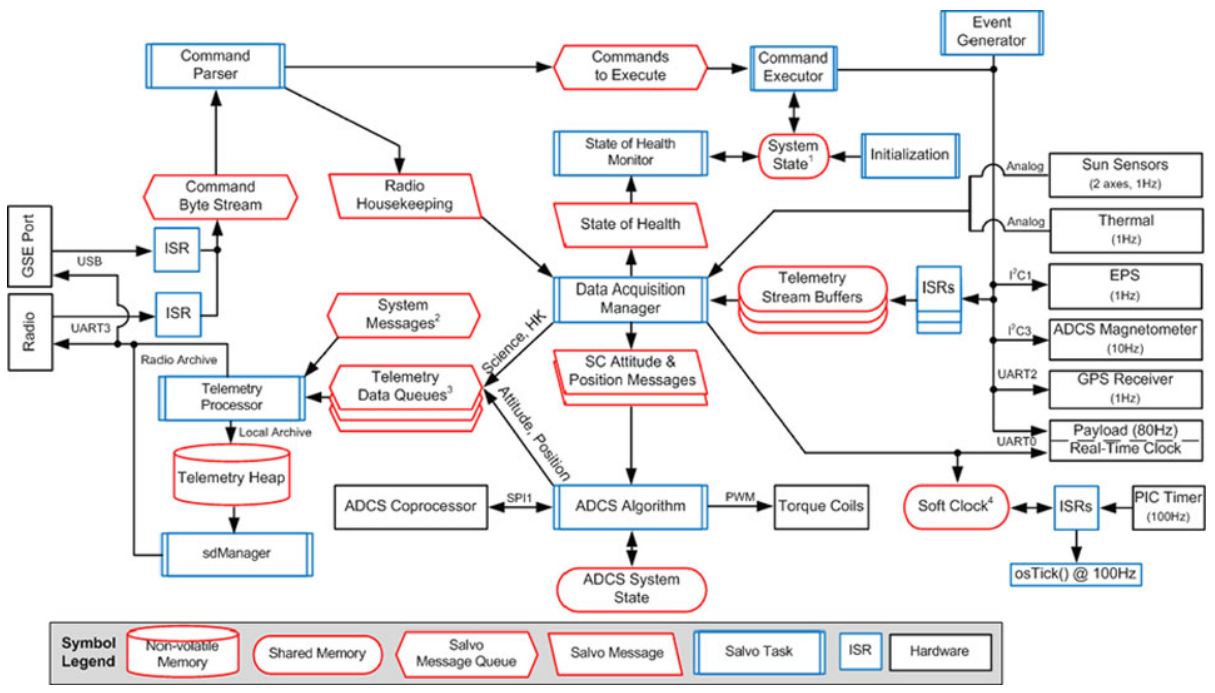

Fig. 25 The DICE FSW is a task-oriented design that communicates via messages and queues

Table 2 DICE sensor-sat FSW allowed operating mode functions and processes
Safe Mode
Standby Mode
Operational Mode

(A) Start up to this mode occurs after a system power recycle and FSW reset

(B) Deploy UHF antennas/Langmuir probes after orbit insertion by firing the main release mechanism

(C) Continuously collect ADCS, system and HK telemetry

(D) Sub-systems unnecessary for spacecraft survival and ground communications are powered down (e.g., GPS receiver, science instruments, ADCS torque coils) (E) Transition to Standby mode must be commanded from ground
(A) Continuously collect ADCS, system, and HK telemetry

(B) Power to GPS receiver and torque coils is allowed

(C) Transition to Operational mode must be commanded from ground

(D) HK state of health monitor errors or ground command will return the spacecraft to Safe mode

(E) Transition to Operational mode must be commanded from the ground
(A) Continuously collect science (when engaged), ADCS, system, and HK telemetry

(B) Power to science instruments, GPS receiver, torque coils allowed (C) HK state of health monitor errors will return the spacecraft to Safe mode (D) Transition to Standby mode must be commanded from the ground

Notably, Safe mode is used as the mode to which the spacecraft boots following a power recycle or FSW reset. It is also the mode to which the spacecraft will automatically transition to when HK state of health boundaries (e.g., predefined over voltage, current, and temperature conditions) are exceeded in Standby or Operational modes. At times of nominal operation and system status, the DICE sensor-sat FSW will only transition between modes upon command from the ground. Transitions must be sequential; it cannot transition from Safe to Operational mode without first going through Standby mode; all other commanded transitions are allowed. When in Standby mode, major system features such as GPS and ADCS torque coil can be enabled. Science instrument operation can only be enabled from Operational mode. The science instruments do not initiate after a transition into Op- 
Table 3 DICE sensor-sat science measurement telemetry configuration (@35 Hz)

\begin{tabular}{|c|c|c|c|c|c|c|}
\hline \multirow[t]{2}{*}{ Channel Name } & \multirow{2}{*}{$\begin{array}{l}\text { Rate } \\
(\mathrm{Hz})\end{array}$} & \multirow{2}{*}{$\begin{array}{l}\text { Word Size } \\
\text { (\# bits) }\end{array}$} & \multirow{2}{*}{$\begin{array}{l}\text { Sample Size } \\
\text { (\# Words) }\end{array}$} & \multirow{2}{*}{$\begin{array}{l}\text { Bit Rate } \\
\text { (bits/s) }\end{array}$} & \multicolumn{2}{|c|}{ Sample Period } \\
\hline & & & & & Orbit $^{\mathrm{a}}$ & Spatial $(\mathrm{km})^{\mathrm{b}}$ \\
\hline EF Probe DC Pair 1_2 & 35.00 & 16.00 & 1.00 & 560.00 & 194376.00 & 0.22 \\
\hline EF Probe DC Pair 3_4 & 35.00 & 16.00 & 1.00 & 560.00 & 194376.00 & 0.22 \\
\hline EF Probe AC Wave Power & 1.00 & 16.00 & 4.00 & 64.00 & 5553.60 & 7.70 \\
\hline Floating Potential Probe & 35.00 & 16.00 & 1.00 & 560.00 & 194376.00 & 0.22 \\
\hline Langmuir Probe 1 & 35.00 & 16.00 & 1.00 & 560.00 & 194376.00 & 0.22 \\
\hline Langmuir Probe 2 & 35.00 & 16.00 & 1.00 & 560.00 & 194376.00 & 0.22 \\
\hline Sweeping Probe 1 & 0.01 & 16.00 & 512.00 & 67.99 & 46.09 & 927.71 \\
\hline Sweeping Probe 2 & 0.01 & 16.00 & 512.00 & 67.99 & 46.09 & 927.71 \\
\hline Science Mag $X$-Axis & 35.00 & 18.00 & 1.00 & 630.00 & 194376.00 & 0.22 \\
\hline Science Mag $Y$-Axis & 35.00 & 18.00 & 1.00 & 630.00 & 194376.00 & 0.22 \\
\hline Science Mag Z-Axis & 35.00 & 18.00 & 1.00 & 630.00 & 194376.00 & 0.22 \\
\hline On orbit Rate (bits/s) & $=$ & & & $4889.99^{c}$ & & \\
\hline
\end{tabular}

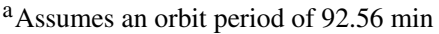

${ }^{b}$ Assumes a spacecraft velocity of $7.7 \mathrm{~km} / \mathrm{s}$

${ }^{\mathrm{c}}$ Does not include packet format overhead
}

erational mode; they can only start while in Operational mode, but a separate sequential ground command is required.

The DICE telemetry is comprised of four main components; science measurements, HK measurements (e.g., voltage, current, and temperature measurements), ADCS measurements (e.g., magnetometer and GPS data), and FSW system state messages (e.g., mode, warning and error messages). The science measurements are only acquired on command when the spacecraft is in Operational mode, and include at regular intervals (1) two EF probe DC differential measurements, (2) an EF probe AC measurement (i.e., Fast Fourier Transform analysis), (3) a spacecraft floating potential measurement, (4) two Langmuir probes DC measurements, (5) two Langmuir probe sweep measurements where the voltage applied to the sensor is swept from -4 to $+1.7 \mathrm{~V} \mathrm{DC}$, and (6) a 3-axis science grate magnetometer measurement set ( $X, Y$, and $Z$ components). Further there are two science measurement data acquisition rates commanded from the ground; a nominal, default " $35 \mathrm{~Hz}$ " rate and a diagnostic " $70 \mathrm{~Hz}$ " rate. The $35 \mathrm{~Hz}$ science telemetry configuration is shown in Table 3 .

The $70 \mathrm{~Hz}$ configuration is identical other than the sampling rate of the signals is increased from 35 to $70 \mathrm{~Hz}$ for those that were at $35 \mathrm{~Hz}$. The other signal sampling rates remain the same between the two configurations. The HK, ADCS, and FSW system state messages telemetry are generated in all sensor-sat modes. Only the HK rates can be changed via ground command, with the ability to be set to rates between 0.1 to $1 \mathrm{~Hz}$. Overall, the instantaneous on-board telemetry rate is $1547 \mathrm{bits} / \mathrm{s}$ when the system is not in science measurement configuration and $\mathrm{HK}$ is set to $0.1 \mathrm{~Hz}$. When the science measurement rate is set to $35 \mathrm{~Hz}$ and $\mathrm{HK}$ is set to $0.1 \mathrm{~Hz}$, the instantaneous on-board telemetry rate increases to $6780 \mathrm{bits} / \mathrm{s}$.

To compile and format telemetry data, the C\&DH collects individual HK, ADCS, and instrument data into records or "granules." The science granules are generated at a rate of $70 \mathrm{~Hz}$ or $35 \mathrm{~Hz}$. The granules are collected in a First-in-First-Out (FIFO) buffer which 


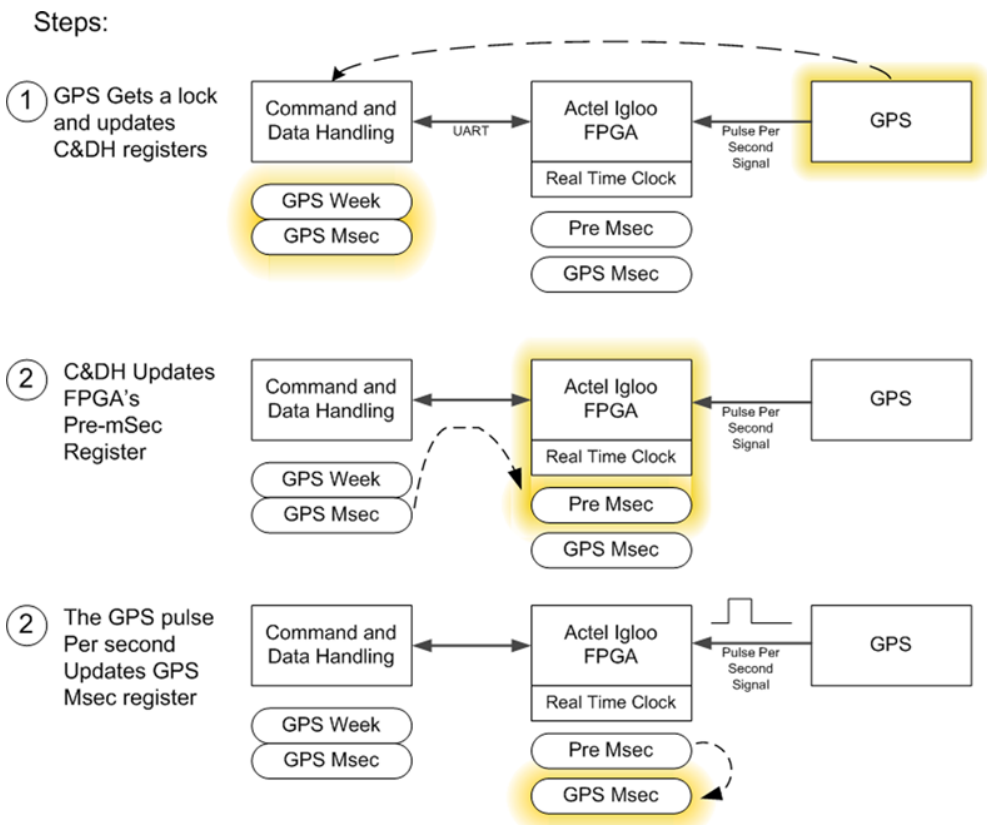

Fig. 26 The DICE real time clock process was developed to maximize time stamp accuracy of the science measurements

are then processed and forward error corrected (FEC) into radio packets for storage on the radio's non-volatile memory. The radio packets conform to the Consultative Committee for Space Data Systems (CCSDS) telemetry specification. The granules form the main body of the CCSDS packet but redundant data is compressed and the granule header is not included to save telemetry space. Because of the linear monotonic nature of the GPS time signal, only the delta time is sent. The first packet header contains the full time stamp, and each subsequent CCSDS packet is referenced from the time header of the first.

Having an accurate clock and being able to associate science instrument data with time is essential for the science measurements. The timestamp enables one to associate the data in a spatial frame and cross referencing it with data from other missions. An incorrect timestamp can affect analysis as it is used for spatial reference; therefore it is important that the clock be as accurate as possible. On DICE, there are two main clock systems. The first is a "soft clock" that runs continuously on the C\&DH system. It is rather sensitive to temperature and can drift on the order of minutes per day. The DICE highly accurate clock is synchronized to the on-board GPS which has a clock with resolution $<1 \mathrm{~ms}$ and accuracy $<20 \mathrm{~ns}$ RMS. The GPS receiver sends out a digital pulse called the pulse per second (PPS) signal. Once the signal is sent the GPS system updates the GPS week and millisecond registers in the C\&DH sub-system. The C\&DH updates a pre-clock register in the timekeeper module of the science FPGA with the value of the next PPS time. Once the PPS pulse is detected the FPGA synchronizes its time with GPS. Dual-rank synchronization in the science FPGA adds between 167 and 250 ns delay when the PPS signal registers the new time. The science FPGA then continues to create relative time stamps from the GPS updates. The science PCB has a very stable ( $<100 \mathrm{~ns} /$ day) oscillator for the relative timer. A flow diagram of this timing process is shown in Figs. 26 and 29. 


\subsubsection{Attitude Determination and Control Sub-system}

ADCS on DICE is handled by both the C\&DH PIC24 microcontroller and a floating point unit (FPU) on the ADCS board. Data is acquired for the ADCS algorithms via the ADCS electronics PCB, and includes 3-axis magnetometer, sun sensor vector, and GPS position and velocity measurements. The torque coil drive circuits are also located on the ADCS electronics PCB. Because the C\&DH PIC24 is inefficient at calculating floating-point numbers, the FPU receives data and instructions from the PIC24, performs the calculations, and returns the results. The magnetometer and sun sensor are sampled by the data acquisition manager task every $10 \mathrm{~Hz}$ to feed the ADCS algorithms. The returned results are then used for pulse-width modulation firing of the torque coils.

The DICE ADCS operates in two states. The first state, Detumble, is entered upon power up of the spacecraft. The spacecraft will remain in this state permanently until commanded from the ground to do otherwise. In Detumble state, based upon magnetometer measurements the spacecraft will "slow" the $X$ and $Y$ axes motion (in regards to the spin rate) and begin to spin up the $Z$ axis in preparation to operate in the Controller state where the spacecraft will maintain the desired spin rate as controlled by ground commands as well as alignment with the geodetic axis. The Detumble state operates by using a modified version of a B-dot algorithm. The local magnetic field vector is sampled by the ADCS magnetometer at $10 \mathrm{~Hz}$. The change in the magnetic field and the required magnetic moment from the magnetic torque coils is calculated:

$$
\begin{aligned}
& \Delta \vec{B}=\vec{B}-\vec{B}_{\text {previous }} \\
& \vec{M}_{\text {detumble }}=-\Delta \vec{B}
\end{aligned}
$$

The B-dot algorithm is modified to avoid decreasing the spin rate about the desired spin axis as this would be an unnecessary step. This is accomplished by calculating the magnetic moment required to create a torque about the desired spin axis.

$$
\begin{aligned}
& \vec{T}=\left[\begin{array}{l}
0 \\
0 \\
1
\end{array}\right] \\
& \vec{M}_{\text {spinup }}=\frac{\vec{B} \times \vec{T}}{|\vec{B}|^{2}}
\end{aligned}
$$

The magnetic moments required to detumble and spinup are normalized and concatenated together with a gain on the detumble magnetic moment.

$$
\vec{M}_{\text {command }}=K_{d} \times\left|\vec{M}_{\text {detumble }}\right|+\left|\vec{M}_{\text {spinup }}\right|
$$

As the gain $K_{d}$ is decreased, more emphasis is placed on spin up. After the DICE satellites detumbled sufficiently to obtain GPS lock, the satellites could be switched to the spin stabilize operational mode. In the spin stabilize mode, the spin rate of the satellites is increased to $0.1 \mathrm{~Hz}$. This is accomplished by decreasing the gain $K_{d}$ thus allowing the spin rate of the satellite to increase.

Upon receiving command from the ground, DICE enters into its Controller state. Sun sensor and magnetometer measurements are used in the Controller state. The interaction of inputs, outputs, and ADCS algorithms for the Controller state is shown in Fig. 27. 
Fig. 27 Data flow and events diagram for the DICE ADCS Controller state

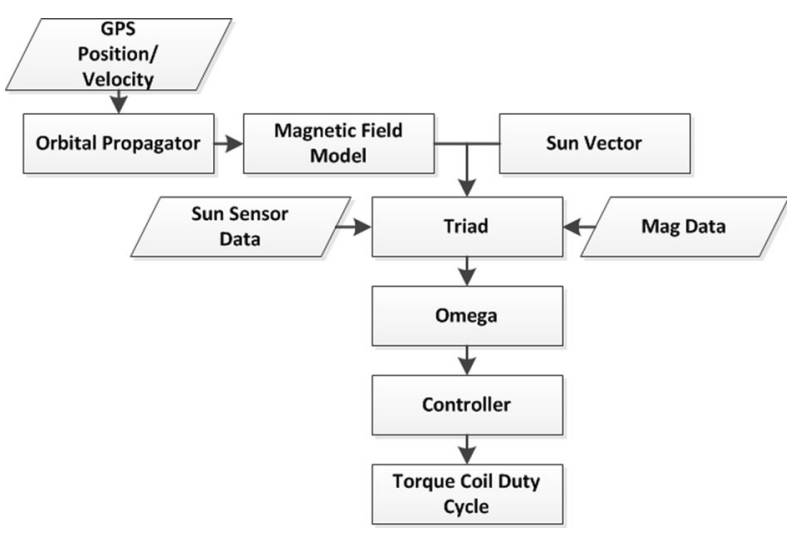

Once the satellite is in a stable spin, the spin axis of the satellite can be rotated to align with the Earth's nominal rotational axis. This algorithm requires inputs from the sun sensor and as such can only be used when the satellites in sunlight. A number of support algorithms are required to successfully align the spin axis, including triad, angular velocity estimation, sun vector, magnetic field, onboard orbit propagator, and spin axis alignment control algorithms. The triad algorithm is used to calculate the attitude of the satellite in the form of a direction cosine matrix. The triad algorithm accepts inputs from the magnetometer $\left(\vec{B}^{b}\right)$, the sun sensor $\left(\hat{s}^{b}\right)$, a magnetic field model $\left(\vec{B}^{i}\right)$, and a sun vector model $\left(\hat{s}^{i}\right)$. Six basis unit vectors are created using the following equations where $(x)$ is $(b)$ or $(i)$ depending upon whether the input is from the sensors or the models.

$$
\begin{aligned}
& \hat{i}_{1}^{x}=\hat{s}^{x} \\
& \hat{i}_{2}^{x}=\frac{\hat{s}^{x} \times \vec{B}^{x}}{\left|\hat{s}^{x} \times \vec{B}^{x}\right|} \\
& \hat{i}_{3}^{x}=\hat{i}_{1}^{x} \times \hat{i}_{2}^{x}
\end{aligned}
$$

The basis vectors are used to create two direction cosine matrices and the attitude of the satellite:

$$
R_{i}^{b}=\left[\begin{array}{lll}
\hat{i}_{1}^{b} & \hat{i}_{2}^{b} & \hat{i}_{3}^{b}
\end{array}\right]\left[\begin{array}{lll}
\hat{i}_{1}^{i} & \hat{i}_{2}^{i} & \hat{i}_{3}^{i}
\end{array}\right]^{-1}
$$

The angular velocity of the satellite is calculated using the current and previously calculated attitude direction cosine matrix. A direction cosine matrix that describes the rotation of the satellite from its previous attitude to its current attitude can be calculated as follows:

$$
R_{\text {previous } \rightarrow \text { current }}=\left[R_{i}^{b}\right]_{\text {current }} *\left[R_{i}^{b}\right]_{\text {previous }}^{-1}
$$

If the rotation between the previous and current times is assumed to be small, the directions cosine matrix can be simplified as follows:

$$
R_{\text {previous } \rightarrow \text { current }} \approx\left[\begin{array}{ccc}
1 & \Delta \theta_{z} & -\Delta \theta_{y} \\
-\Delta \theta_{z} & 1 & \Delta \theta_{x} \\
\Delta \theta_{y} & -\Delta \theta_{x} & 1
\end{array}\right]
$$


This simplification reduces the rotation matrix to small angular changes about the satellite $X, Y$, and $Z$ axes. The angular velocity of the satellite can be calculated by approximating the time derivative of these small angles by:

$$
\vec{\omega}_{b / i}^{b} \approx\left[\begin{array}{c}
\frac{\Delta \theta_{x}}{\Delta \mathrm{t}} \\
\frac{\Delta \theta_{y}}{\Delta \mathrm{t}} \\
\frac{\Delta \theta_{z}}{\Delta \mathrm{t}}
\end{array}\right]
$$

A simple sun vector algorithm is used to calculate the expected location of the sun in the J2000 inertial coordinate system (Vallado 2001), and accepts the current Modified Julian Date as an input, and outputs the expected unit vector to the sun. The Modified Julian Date is calculated from GPS time when GPS is available; otherwise it is calculated using the onboard clock. The expected local magnetic field vector is calculated using the 2010 World Magnetic Model (WMM). The WMM 2010 is reduced to a simple dipole model for use onboard the DICE sensor-sats. The WMM requires the satellites current position as an input. The position of the satellite is obtained from the GPS receiver when GPS is available; otherwise the position of the satellite is calculated using an onboard orbit propagator. The orbit propagator on the DICE satellites is a simple two-body orbit propagator. The algorithm directly integrates the two-body equations of motion using a 4th order Runge-Kutta integrator:

$$
\begin{aligned}
& \dot{\vec{r}}^{i}=\dot{\vec{v}}^{i} \\
& \dot{\vec{v}}^{i}=-\frac{\mu_{\text {earth }}}{\left|\vec{r}^{i}\right|^{3}} * \vec{r}^{i}
\end{aligned}
$$

The spin axis of the satellite is aligned by controlling the angular momentum vector of the satellite. The angular momentum of the satellite in a satellite body coordinate system is approximated by assuming that the body coordinate system is aligned with the principal axes of inertia:

$$
\vec{h}^{b} \approx\left[\begin{array}{c}
I_{x x}^{b} * \omega_{x}^{b} \\
I_{y y}^{b} * \omega_{y}^{b} \\
I_{z z}^{b} * \omega_{z}^{b}
\end{array}\right]
$$

The angular momentum of the satellite is rotated to the inertial J2000 coordinate system using the direction cosine matrix calculated using the triad algorithm:

$$
\vec{h}^{i}=R_{b}^{i} * \vec{h}^{b}
$$

The torque required to align the spin axis and maintain the current spin rate is calculated in the inertial coordinate system and then rotated back to the satellite body coordinate system:

$$
\begin{aligned}
& \vec{T}_{\text {align }}^{i}=-K_{h} *\left[\begin{array}{c}
h_{x}^{i} \\
h_{y}^{i} \\
h_{z}^{i}-I_{z z}^{b} * \omega_{\text {desired }}
\end{array}\right] \\
& \vec{T}_{\text {align }}^{b}=R_{i}^{b} * \vec{T}_{\text {align }}^{i}
\end{aligned}
$$


The torque required to dampen the nutation of the satellite is also calculated in the body coordinate system of the satellite:

$$
\vec{T}_{\text {nutation }}^{b}=\left[\begin{array}{c}
-K_{x} * \omega_{x}^{b} \\
-K_{x} * \omega_{y}^{b} \\
-K_{z} *\left(\omega_{z}^{b}-\omega_{\text {desired }}\right)
\end{array}\right]
$$

The alignment torque and the nutation torque are concatenated and the magnetic moment required to generate this torque is calculated:

$$
\begin{aligned}
\vec{T}_{\text {command }} & =\vec{T}_{\text {align }}^{b}+\vec{T}_{\text {nutation }}^{b} \\
\vec{M}_{\text {command }} & =\frac{\vec{B} \times \vec{T}_{\text {command }}}{|\vec{B}|^{2}}
\end{aligned}
$$

After the spin axis has been successfully aligned, the spin rate of the satellite is increased to allow for boom deployment. This is accomplished by increasing the desired spin rate $\left(\omega_{\text {desired }}\right)$ in the spin axis alignment algorithm. During boom deployment, the spin axis alignment algorithm continues to maintain the satellite alignment and spin rate. As the booms are deployed, the spin rate decreases, but the satellite spin rate increases to continue deployment. The process continues until the booms are completely deployed.

Each DICE spacecraft carries a NovAtel OEMV-1 GPS receiver along with a commercial L1 patch antenna placed on the $+Z$ top place of the spacecraft. As the spacecraft spins about its $Z$ axis at up to $2 \mathrm{~Hz}$, the patch antenna rotates but maintains the same GPS constellation vehicles in view for several minutes allowing the receiver to acquire the signals long enough to produce a position and velocity solution. Due to the $\sim 1 \mathrm{~W}$ power consumption of the GPS, it is only powered on periodically (nominally every 3 hours) and powered down after the solution is acquired.

Pre-flight testing of the OEMV-1 using the NASA Goddard Space Flight Center GPS simulator was performed using a variety of spacecraft attitude scenarios including insertion tumbling after deployment, insertion orbit $0.1 \mathrm{~Hz}$ and $1 \mathrm{~Hz}$ spin with geodetic axis alignment, as well as end of life orbit $1 \mathrm{~Hz}$ spin with geodetic axis alignment. While acquiring a consistent lock and position solution in the tumble scenario was challenging, consistent locks could be made in all other scenarios regardless of spacecraft altitude or spin rate, with time-to-lock from cold start ranging from 75 to 810 seconds and an average of 401 seconds. An interesting correlation was observed for all scenarios involving spin about the spacecraft axis, when it was aligned to the geodetic axis. Locks tended to acquire more quickly when cold start was initiated as the spacecraft entered low latitudes (lower than -45 degrees) as shown in Fig. 28.

DICE implements a precise clock which tracks real time to $<90 \mathrm{~ns}$ precision in the science payload electronics FPG(A) Clock drift is corrected and synchronized by the C\&DH whenever the GPS receiver is powered on and producing precise time solutions, as shown in Fig. 29. The DICE-time synchronization scheme allows the payload clock to be synchronized to the GPS receiver's clock to within $250 \mathrm{~ns}$ or within $270 \mathrm{~ns}$ of real time. Emulated GPS time stamps can also be sent from the ground to the spacecraft during mission operation times in which the GPS is not powered on and acquiring real GPS time, position, and velocity. Time accuracy is degraded during these times. 


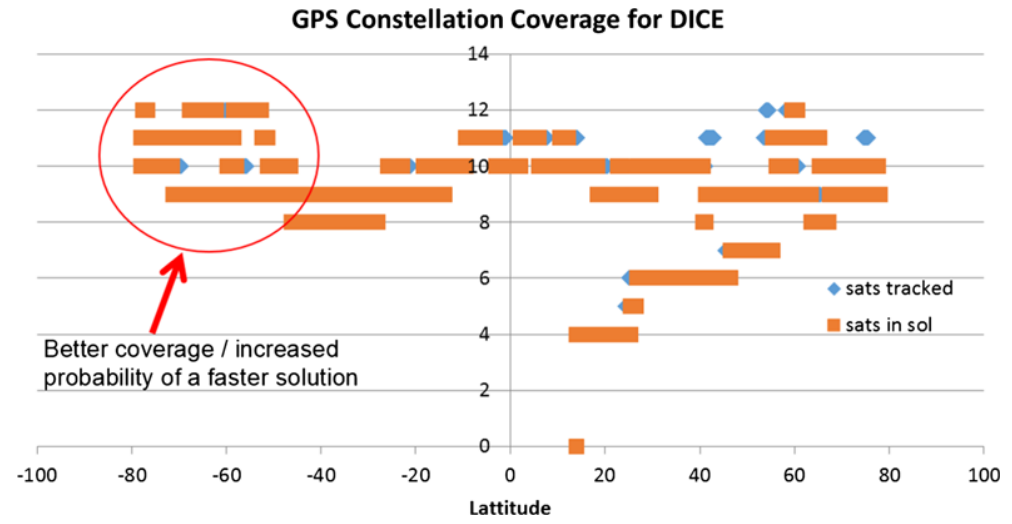

Fig. 28 GPS simulation test data from NASA Goddard; in this data set, the DICE spacecraft were aligned with the geodetic axis and spinning

Fig. 29 The DICE system timing is based on a combination of clocks that are ultimately synchronized to GPS time

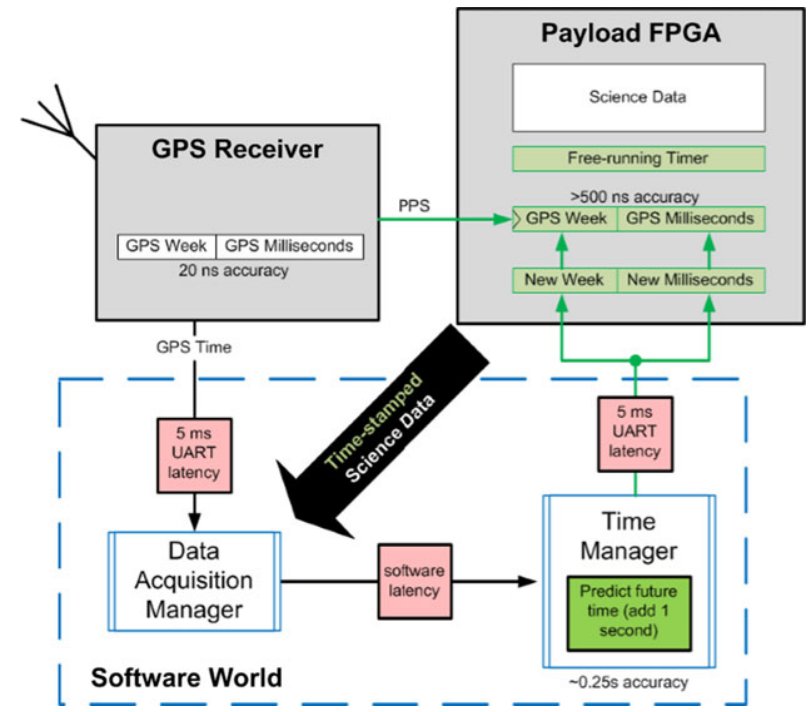

\subsubsection{Structural and Thermal Sub-systems}

The DICE sensor-sat design used judicious material selection and load-spreading to withstand launch loads. The frame was fabricated from a single block of Aluminum 7075, using a wire-EDM machine process, which provided added strength above the typical Aluminum 6061 material as well as eliminated possible vibrational modes typically found in fastenerjoined systems. The rails of the structure were hard-anodized per the Cal Poly CubeSat specifications. A webbing pattern was used in the structural design, minimizing mass while still maintaining the strength necessary to meet structural requirements. Structural analysis was performed using finite element analysis modeling software (reference Fig. 30). The DICE sensor-sat structure is fairly rigid body and has a fundamental frequency of $\sim 203 \mathrm{~Hz}$.

Given their small volume, the DICE sensor-sats possess very little thermal inertia. To address this and the expected resultant on-orbit thermal swings of 50 to $100^{\circ}$ per orbit, the 
Fig. 30 FEMAP model of the

DICE sensor-sats

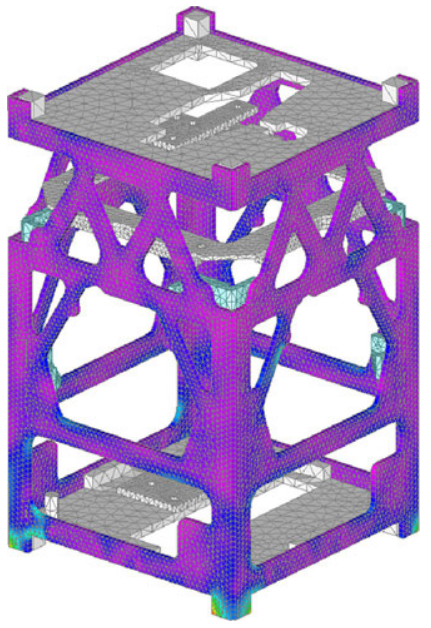

thermal conductivity between the outer solar panels and the spacecraft structure was minimized. This simple thermal segregation design approach helped minimize internal thermal swings. As the electronics operate within the structure, the thermal generation of the boards themselves provide a heater system, allowing the sensor-sats to stay within functional temperature ranges while the solar panels may swing by $100^{\circ}$ from one orbit to the next. This effect is shown in the on-orbit thermal housekeeping data shown in Fig. 43. The upper left panel shows internal electronic temperatures, with orbit to orbit changes on the order of 10 to $15^{\circ}$, while the upper left panel shows much greater temperature variation on the solar panels.

The bottom panel of each spacecraft was chosen to act as the radiator, both because of its proximity to the radio on the electronics stackup and as that panel stares into space for a good portion of each DICE orbit. To further increase the radiating capabilities of the panel, aluminized tape was placed on all of the exposed surface of the panel. This tape allowed the panel to act as a radiator while also reflecting albedo and energy from the sun that would cause the radiator to heat up. The final taped plate is shown in Fig. 31.

\subsubsection{Communication Sub-system}

The total on-board formatted data acquisition rate of each DICE sensor-sat is $>6.8 \mathrm{kBits} / \mathrm{s}$ when in $35 \mathrm{~Hz}$ science mode ( $11.8 \mathrm{kBits} / \mathrm{s}$ in $70 \mathrm{~Hz}$ mode). To store and forward transmit this data to the ground from the DICE constellation on a daily cadence requires an on-board storage of $\sim 1 \mathrm{Gbit} /$ day and a downlink rate greater than $1.5 \mathrm{Mbits} / \mathrm{s}$ (assumes approximately 7-10 minutes of overpass downlink time per sensor-sat per day). The on-board storage requirement is well within typical CubeSat technology specifications. However, the downlink rates are much greater (by a factor of 100-200) than those used on most previous CubeSat missions. Prior CubeSat missions have typically been "beacon" communicators that relied on amateur radio frequencies for downlink communications as well as enthusiastic amateur radio operator interest to track the spacecraft, acquire the beacon data, and then distribute the mission data to those interested. Additionally, as DICE was sponsored by NSF and NASA it is inherently a government program that should use government sponsored communication bands. 
Fig. 31 Aluminized tape on DICE plate used to reflect heat

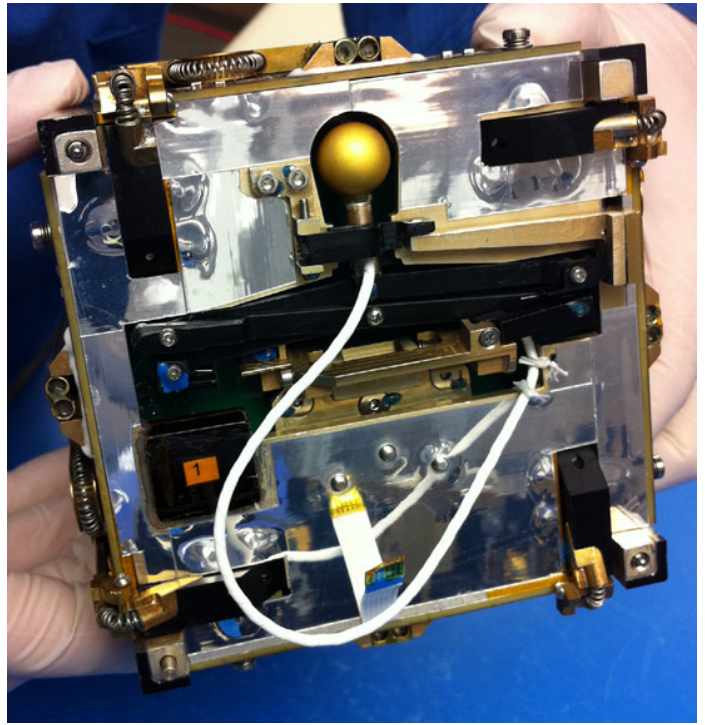

Fig. 32 L-3 Comm's CadetU miniature radio is ideal for CubeSats and small satellites

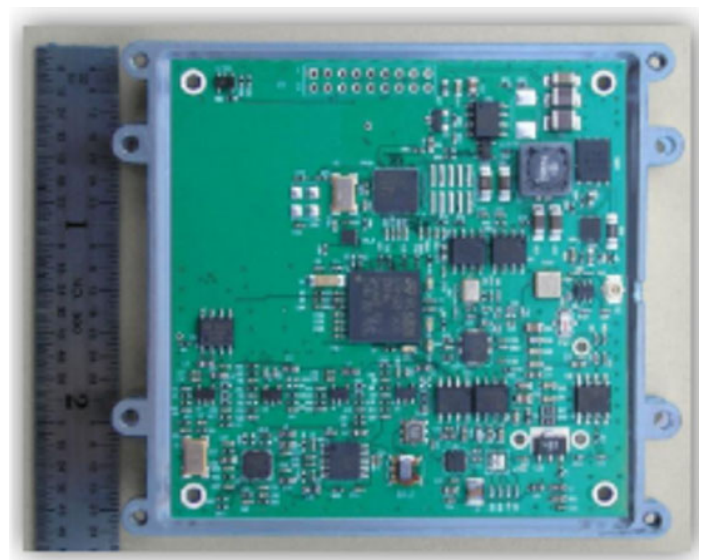

Therefore, in collaboration with L-3 Communications, the DICE team developed the CadetU CubeSat radio to enable high downlink data rates and to operate in government assigned and regulated UHF bands for both uplink and downlink communications. The use of UHF bands avoids congested S-band operations, and provides better signal to noise levels over the same communication path. Each DICE sensor-sat contains a CadetU radio. The DICE ground station uses a GNU Radio based software defined radio. A software defined radio (often referred to as SDR) is a radio communication system where components that have been typically implemented in RF hardware are instead implemented by means of software on a personal computer or embedded system. The CadetU is a $6.9 \times 6.9 \times 1.3 \mathrm{~cm}$ half-duplex modem (see Fig. 32), weighing only $0.07 \mathrm{~kg}$, and capable of $9600 \mathrm{kbits} / \mathrm{s}$ uplink (450 MHz) and $20 \mathrm{Mbits} / \mathrm{s}$ of FEC encoded downlink (460-470 MHz). The DICE CadetU radios downlink at $3 \mathrm{Mbits} / \mathrm{s}$ as that is more than ample bandwidth for the mission telemetry needs. The uplink is FSK modulated, while the downlink is OQPSK modulated. The CadetU implements a store-and-forward architecture where data is continuously streamed to it from 


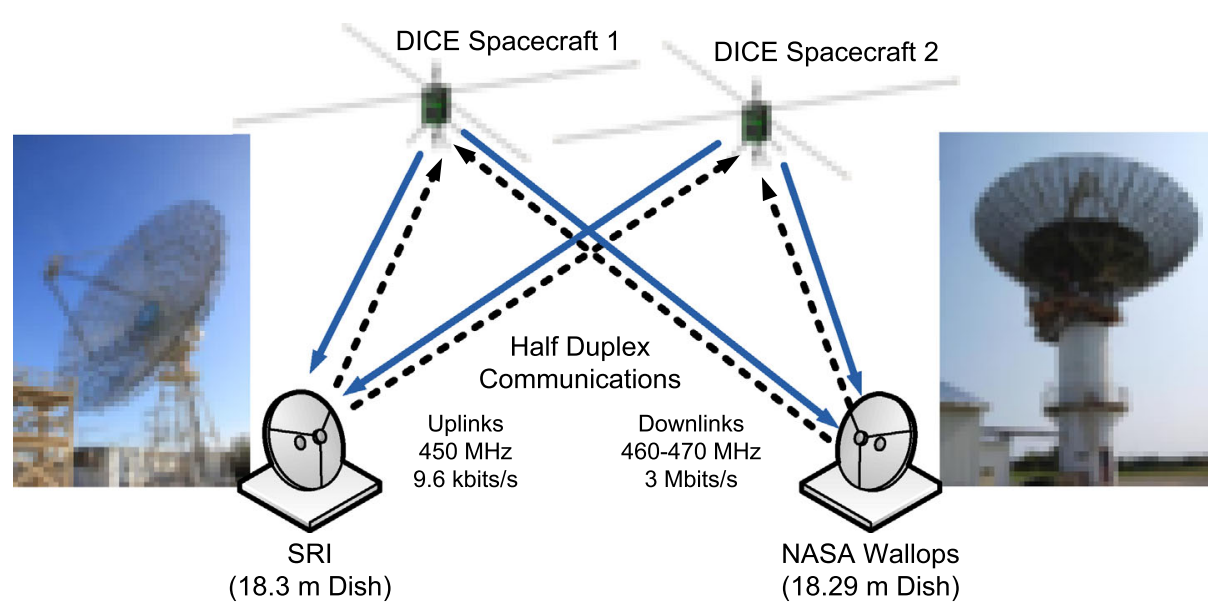

Fig. 33 The DICE communications scenario uses the large UHF dishes at WFF and SRI to close the DICE high speed downlink

the $\mathrm{C} \& \mathrm{DH}$ through a simple UART. It is then pre-encoded and stored in on-board 4 GByte FLASH memory. Ground station operators have control over data transmission, data priority order, and how and when that data is deleted. Continuous receive operations consume $\sim 200 \mathrm{~mW}$, while transmission consumes $\sim 9 \mathrm{~W}$ of power to produce up to $2 \mathrm{~W}$ of RF output power. Output power levels are selectable in $3 \mathrm{~dB}$ increments. The uplink and downlink frequencies can be modified via ground commands, commands with the uplink having a frequency resolution of $10 \mathrm{kHz}$ and the downlink having a resolution of $1 \mathrm{MHz}$. The CadetU implements a custom command protocol which is essentially identical to the front end (RF from ground) or back end (UART to C\&DH). The DICE spacecraft communicate with the ground stations using the CCSDS space packet protocol.

The DICE ground station uses an open-source software defined radio. Uplink commands and data requests are sent through a TI CC1101-based transmitter. Downlinks from the CadetU are captured by the Ettus USRP2 which is hardware that has been specialized for software defined radios. These devices interface through a standard gigabit Ethernet NIC. Using GNU Radio, an open-source software readily available to the public, an interface is created and used to store data from the transmission at $10 \mathrm{Msamples} / \mathrm{s}$. Upon completion of an overpass, data is run through a series of signal processing, demodulation, and FEC decoding scripts before final ingesting into the data center database as level 0 data.

To close the high speed downlink, the primary DICE mission ground station (GS) located at NASA Wallops Flight Facility (WFF), uses the software-defined radio equipment in combination with the WFF $20 \mathrm{~m}$ ( $\sim 35 \mathrm{~dB}$ gain) UHF dish. The secondary GS node is located at SRI International and uses a second DICE software-defined radio in combination with their $20 \mathrm{~m}$ UHF dish. The DICE communications scenario schematic is shown in Fig. 33. The DICE link budget details are shown in Table 4. The link budget is given for worst case scenarios of downlink lock at apogee for $10^{\circ}$ above the horizon. Even for this condition, the downlink demonstrates a total margin of $6.7 \mathrm{~dB}$ (required margin plus margin). The uplink has $>30 \mathrm{~dB}$ of total margin. 
Table 4 DICE sensor-sat link budget

\begin{tabular}{|c|c|c|c|c|}
\hline Design Element & Symbol & Units & $E$ to $S$ & $\mathrm{~S}$ to $\mathrm{E}$ \\
\hline Link Frequency & $f$ & $\mathrm{GHz}$ & 0.5 & 0.5 \\
\hline Transmitter Power & $P_{t x}$ & Watts & 16.0 & 2.0 \\
\hline Transmitter Power & $P_{t x}$ & $\mathrm{dBW}$ & 12.0 & 3.0 \\
\hline \multicolumn{5}{|l|}{ Transmitter } \\
\hline Antenna Gain & $G_{t x}$ & $\mathrm{~dB}$ & 36.5 & -5.0 \\
\hline Antenna Transmitter Losses & $L_{t x}$ & $\mathrm{~dB}$ & -0.5 & -0.5 \\
\hline Antenna Beam Width & $q_{t x}$ & Deg & 2.3 & 180.0 \\
\hline Antenna Misalignment & $a_{t x}$ & Deg & 0.5 & 0.0 \\
\hline Alignment Loss & $L_{q t x}$ & $\mathrm{~dB}$ & -0.6 & 0.0 \\
\hline Equivalent Isotropic Radiated Power & EIRP & $\mathrm{dBW}$ & 47.5 & -2.5 \\
\hline \multicolumn{5}{|l|}{ Losses } \\
\hline Propagation Path Length & $S$ & $\mathrm{~km}$ & 2366.0 & 2366.0 \\
\hline Space Loss & $L_{s}$ & $\mathrm{~dB}$ & -153.0 & -153.3 \\
\hline Atmospheric & $L_{a}$ & $\mathrm{~dB}$ & -0.5 & -0.5 \\
\hline Polarization Loss & $L_{p}$ & $\mathrm{~dB}$ & -1.0 & -1.0 \\
\hline Total Losses & $L$ & $\mathrm{~dB}$ & -154.5 & -154.8 \\
\hline \multicolumn{5}{|l|}{ Receiver } \\
\hline Antenna Gain & $G_{r}$ & $\mathrm{~dB}$ & -5.0 & 35.8 \\
\hline Antenna Receiver Loss & $L_{r}$ & $\mathrm{~dB}$ & -0.5 & -0.5 \\
\hline Antenna Beam Width & $q_{r}$ & Deg & 180.0 & 2.3 \\
\hline Antenna Misalignment & $a_{r}$ & Deg & 0.0 & 0.5 \\
\hline Alignment Loss & $L_{q r}$ & $\mathrm{~dB}$ & 0.0 & -0.6 \\
\hline Total Receiver & $G$ & $\mathrm{~dB}$ & -5.5 & 34.7 \\
\hline Sky (Antenna) Noise Temperature & $T_{a}$ & $\mathrm{~K}$ & 300.0 & 200.0 \\
\hline Receiver Temperature & $T_{r}$ & $\mathrm{~K}$ & 870.0 & 300.0 \\
\hline System Noise Temperature & $T_{S}$ & $\mathrm{~K}$ & 1170.0 & 500.0 \\
\hline Receiver Merit & $G / T$ & $\mathrm{DB}(1 / \mathrm{K})$ & -36.2 & 7.7 \\
\hline \multicolumn{5}{|l|}{ Powers } \\
\hline Power Flux Density & $f$ & $\mathrm{~dB}\left(\mathrm{~W} / \mathrm{m}^{2}\right)$ & -91.0 & -141.0 \\
\hline Occupied Bandwidth & & $\mathrm{kHz}$ & 10.0 & 3000.0 \\
\hline Power Flux Spectral Density & $f_{f}$ & $\mathrm{~dB}\left(\mathrm{~W} / \mathrm{m}^{2} / \mathrm{kHz}\right)$ & -100.8 & -175.7 \\
\hline Power Flux Spectral Density Limit & $f_{f}$ & $\mathrm{~dB}\left(\mathrm{~W} / \mathrm{m}^{2} / \mathrm{kHz}\right)$ & 0.0 & -158.0 \\
\hline Carrier Power Received & $P_{r x}$ & $\mathrm{dBW}$ & -112.5 & -122.5 \\
\hline Noise Spectral Density & $N_{o}$ & $\mathrm{~dB}(\mathrm{~W} / \mathrm{Hz})$ & -197.9 & -201.6 \\
\hline Carrier to Noise Density & $P_{r x} / N_{o}$ & $\mathrm{~dB}(\mathrm{~Hz})$ & 85.4 & 79.1 \\
\hline \multicolumn{5}{|l|}{ Rates } \\
\hline Data Rate & $R$ & $\mathrm{~B} / \mathrm{s}$ & 9600.0 & 3000000.0 \\
\hline $\mathrm{Eb} / \mathrm{No}$ & $E_{b} / N_{o}$ & $\mathrm{~dB}$ & 45.6 & 14.3 \\
\hline Required $E_{b} / N_{o}-$ Modulation Format & $E_{b} / N_{o}$ & $\mathrm{~dB}$ & 13.3 & 9.6 \\
\hline Coding Gain & & $\mathrm{dB}$ & 0.0 & 3.0 \\
\hline Implementation Loss & & $\mathrm{dB}$ & 0.0 & 1.0 \\
\hline Required $E_{b} / N_{o}$ & $E_{b} / N_{o}$ & $\mathrm{~dB}$ & 13.3 & 7.6 \\
\hline Required Margin & & $\mathrm{dB}$ & 4.0 & 4.0 \\
\hline Margin & & $\mathrm{dB}$ & 28.3 & 2.7 \\
\hline
\end{tabular}


Fig. 34 The DICE MOC is run by students at USU/SDL

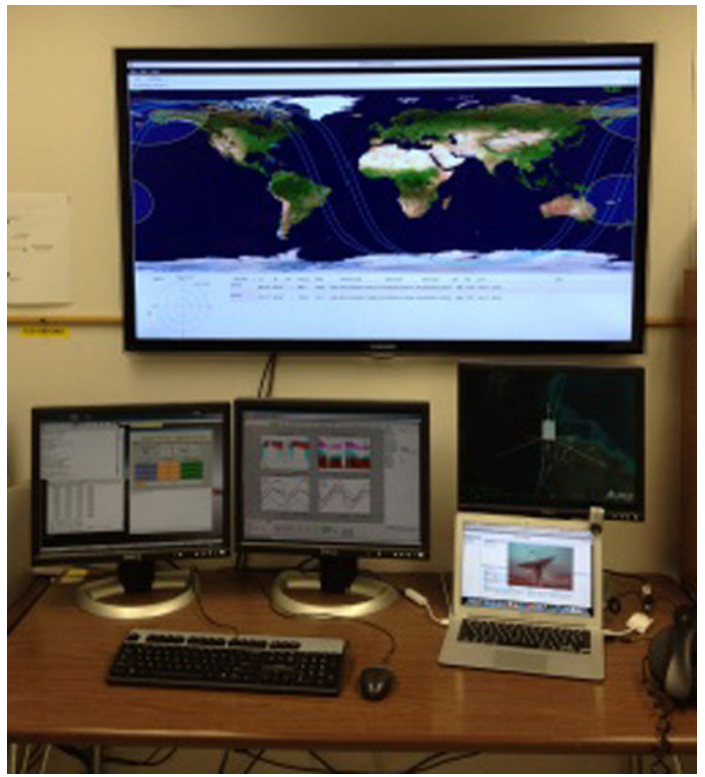

\subsection{Mission Operations and Data Management}

DICE mission operations are designed to be executed by student and professional staff at the USU/SDL mission operations center (MOC), shown in Fig. 34. The MOC is set up to run the WFF and SRI GSs remotely, and coordinate operations with ASTRA. The MOC and GSs rely on the USAF Space Command's published orbital elements for satellite tracking. Spacecraft command and telemetry planning occurs during the week prior to scheduled activity. The USU/SDL MOC is connected to USU/SDL's gigabit intranet, facilitating preprocessing, archiving, and disseminating the mission's data products to the science team. USU/SDL maintains a data center (DC), managed by professionals and staffed by students. GS data acquisitions are ingested into and stored in a MySQL database that contains Level 0 to Level 3 data. The DC is protected against power anomalies, fire, and other adversities. Data stored in the DC is regularly archived and stored at an off-site location. The DC furnishes secure communication for the DICE engineering and science teams, as well as public servers for the dissemination of mission data.

The Level 0-3 processing tasks are outlined in Table 5. DICE spacecraft data is processed to level 0 by the MOC and then transferred to the DC for permanent storage and future access for higher level data product processing by ASTRA, USU/SDL, and EmbryRiddle. ASTRA also validates L0 and L1 data. USU/SDL and Embry-Riddle provide the necessary algorithms for calibration of sensor data. ASTRA runs the Assimilative Mapping of Ionospheric Electrodynamics (AMIE) to drive the Thermosphere Ionosphere Mesosphere Electrodynamic General Circulation Model (TIMEGCM) to develop an estimate of the disturbance dynamo contribution, and an assimilative model called Ionospheric Data Assimilation Algorithm 4-Dimensional (IDA4D) that ingests many different kinds of electron density-related data (Bust et al. 2004) to produce the 3-D electron density distributions. The model output provides a framework for the interpretation of the DICE data (Crowley et al. 2003). AMIE and IDA4D run both with and without the DICE data, for comparison. ASTRA is the public interface to the DICE higher level products. 
Table 5 DICE data products

\begin{tabular}{|c|c|c|}
\hline Data Product & Public Access Location & Data Elements \\
\hline L0 & $\begin{array}{l}\text { USU/SDL } \\
\text { ASTRA } \\
\text { [MOC/DC] }\end{array}$ & $\begin{array}{l}\text { - Removal of duplicate data } \\
\text { - Separation of bus \& science data } \\
\text { - Correlation of system \& absolute time }\end{array}$ \\
\hline L1 & $\begin{array}{l}\text { USU/SDL } \\
\text { ASTRA } \\
\text { [MOC/DC] }\end{array}$ & $\begin{array}{l}\text { - Application of sensor calibration } \\
\text { - Insertion of orbital elements into data } \\
\text { - Calculation of time/geodetic position }\end{array}$ \\
\hline L3 & ASTRA & $\begin{array}{l}\text { For selected storms: } \\
\text { - AMIE potential distribution } \\
\text { - TIMEGCM fields (Ne, E-field, winds) } \\
\text { - IDA4D Ne distribution }\end{array}$ \\
\hline Archive & $\begin{array}{l}\text { USU/SDL } \\
\text { [MOC/DC] }\end{array}$ & - Permanent archival of all mission data \\
\hline
\end{tabular}

Fig. 35 DICE structural random vibration and sinusoidal survey testing

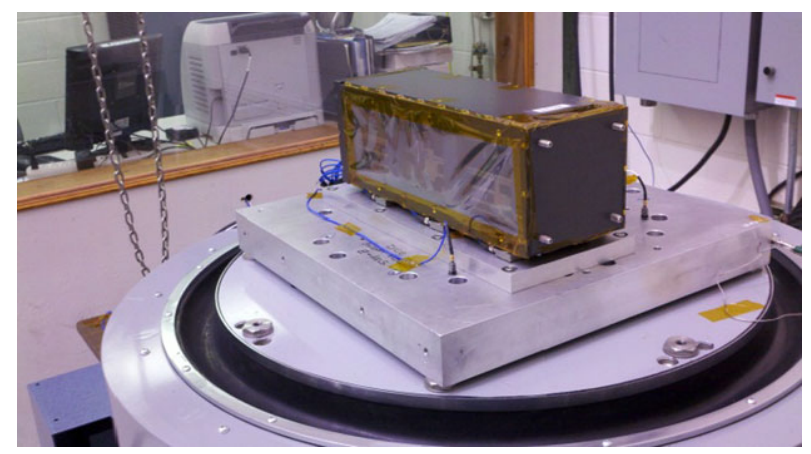

\section{Sensor-Sat Test and Calibration}

\subsection{Environmental and Functional Testing}

As part of the NASA ELaNa III program, the DICE spacecraft were required to undergo environmental qualification and launch specific functional testing. This testing focused on P-POD integrated testing and verification. Essentially, the integrated DICE P-POD assembly was required to demonstrate that there would not be any radio frequency, electrical power, or structural interference between DICE and the host launch vehicle during launch as well as approximately one hour following ejection of DICE from the P-POD. Environmental testing included (1) structural random vibration, sinusoidal survey, and shock, and (2) thermal cycling and thermal vacuum bakeout. The majority of the environmental testing occurred at USU/SDL (see Fig. 35), however to perform the structural shock testing ATK shock test facilities located near Promontory, Utah were used.

Non-ELaNa mission functional testing included "day in the life" operations verification, communication link budget and antenna pattern verification, mass properties verification, ADCS algorithm performance verification, and EPS performance verification testing. The 


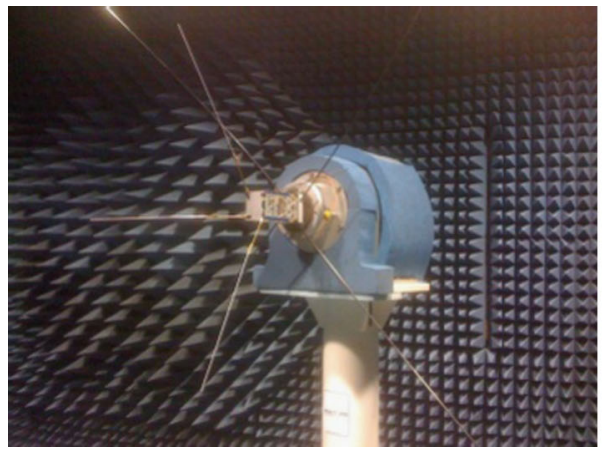

(a)

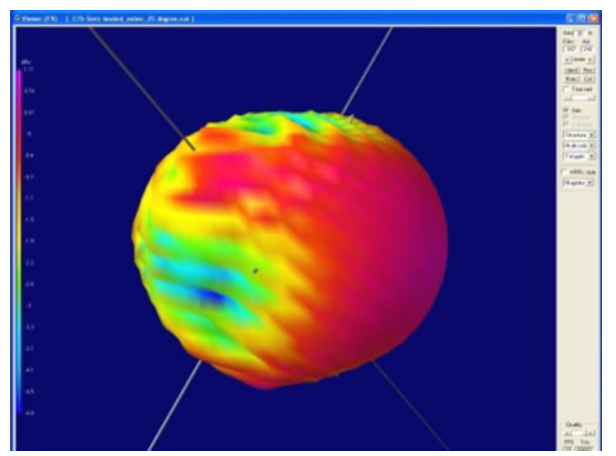

(b)

Fig. 36 (a) The DICE antenna testing at NASA WFF; (b) The resultant antenna pattern is fairly uniform over all view angles (pattern is from a simulated model that closely matches the WFF test data), where the highest gain areas (pink) are $1.1 \mathrm{dBi}$ and the lowest gain areas (dark blue) are $-4.8 \mathrm{dBi}$
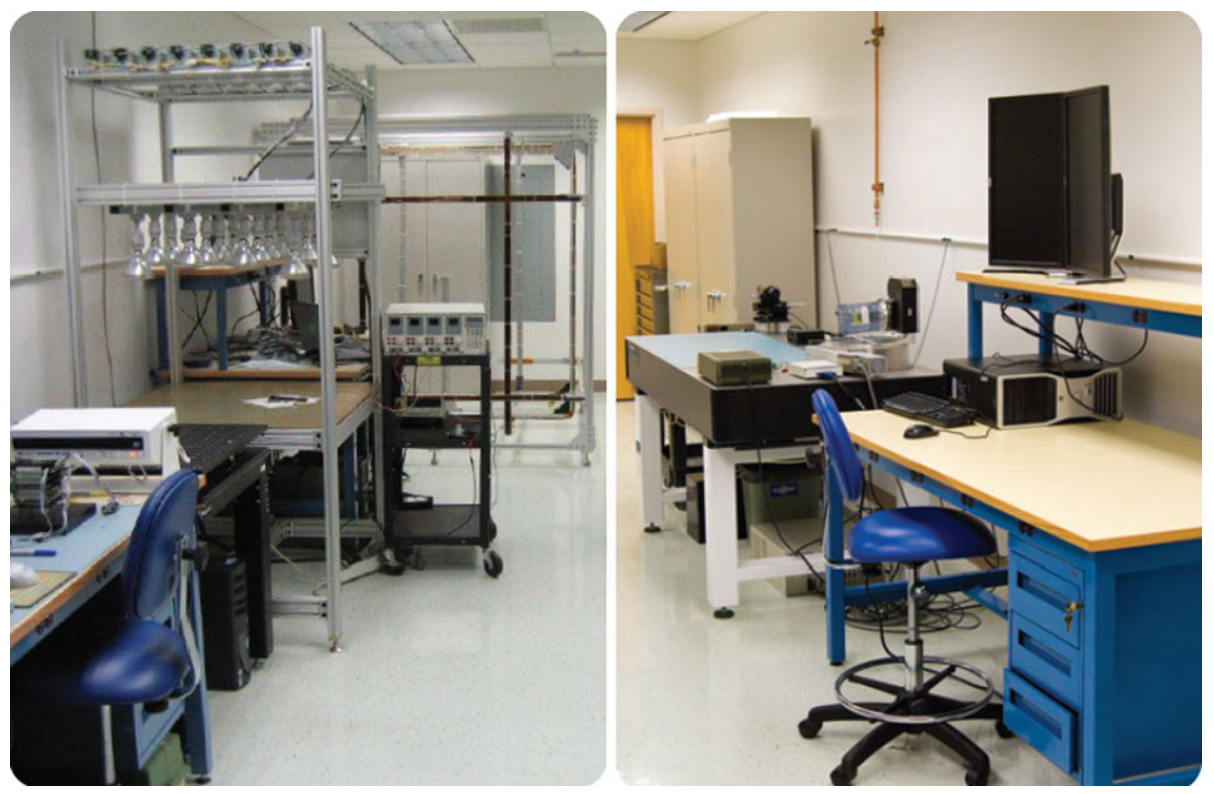

Fig. 37 The USU/SDL NOVA test facility is specially designed to allow for easy access and quick turnaround for CubeSat sized functional testing and system performance calibration activities

communication link budget and antenna pattern verification occurred in collaboration with and at NASA WFF (see Fig. 36).

Additionally, USU/SDL has recently developed the Nano-satellite Operation Verification and Assessment (NOVA) facility (see Fig. 37), which was critical to the functional and mass properties testing on DICE. The NOVA facility includes access to a walk-in Helmholtz cage, NIST traceable solar simulator, inertia and mass properties tables, Gauss chamber, and other hardware-in-the-loop utilities. This CubeSat-targeted test facility, along with the relative 
Fig. 38 Calibration data residuals from triangle wave inputs to EFP

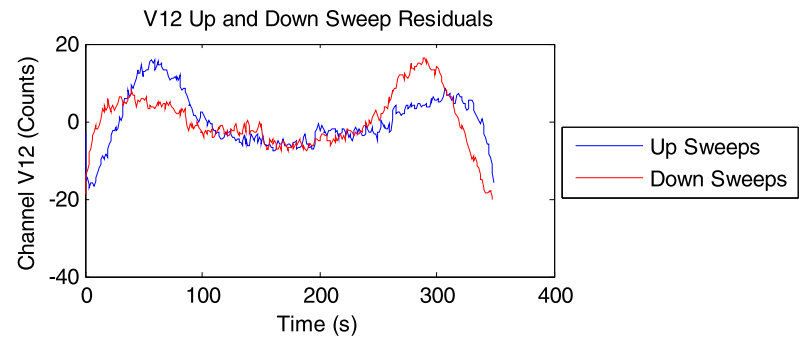

ease in handling CubeSat sized spacecraft, enabled quick turnaround testing and verification throughout the program.

\subsection{Calibration}

Calibration of the science sensors was accomplished by examining the response of the sensors to known loads and test conditions. These measurements and analyses were performed both for bench-test scenarios and while the sensors were undergoing thermal vacuum testing. Engineering quantities are related to digital counts by a set of polynomial coefficients according to

$$
y=A_{0}+A_{1} x+A_{2} x^{2}+\cdots
$$

where $y$ is the calibrated quantity (volts, amperes, degrees, etc.) and $x$ is the telemetered integer quantity. Several of the calibrations were found to depend significantly on temperature; so the coefficients, $A_{i}$, will vary according to temperature. Therefore, the conversion from counts to engineering units for these values is a two-step process. First, the $A$ coefficients are obtained by using the appropriate housekeeping temperature monitor according to

$$
\begin{aligned}
& A_{0}=b_{0}+m_{0} T \\
& A_{1}=b_{1}+m_{1} T
\end{aligned}
$$

where $T$ is the measured temperature, and the coefficients $b$ and $m$ are determined during thermal vacuum testing and calibration. Once the coefficients $A$ are determined, then the conversion from counts $(x)$ to engineering quantities $(y)$ is performed.

The electric field sensor was treated as a voltmeter. A stable voltage source was applied between the differential probe surfaces and measured with a calibrated voltmeter. The measured voltage and the telemetered counts were used to obtain the correct calibration coefficients. The magnetometer was calibrated in a characterized Helmhotz coil located within a Gauss chamber, with triple-walled Mu-metal. The Helmhotz coil was driven with known currents as measured by a calibrated ammeter. The Langmuir probes were treated as ammeters and varying known resistive loads were applied to their sensor outputs. The measured current readings and the telemetered counts were used to obtain the correct calibration coefficients. These calibrations were performed over a range of temperatures during bench top and thermal chamber testing. Figure 38 is an example plot of the residuals of a linear fit from the calibration process of electric field data. Figure 39 shows plots of the nine elements the final calibration matrix for the 3 -axis SciMag data. 

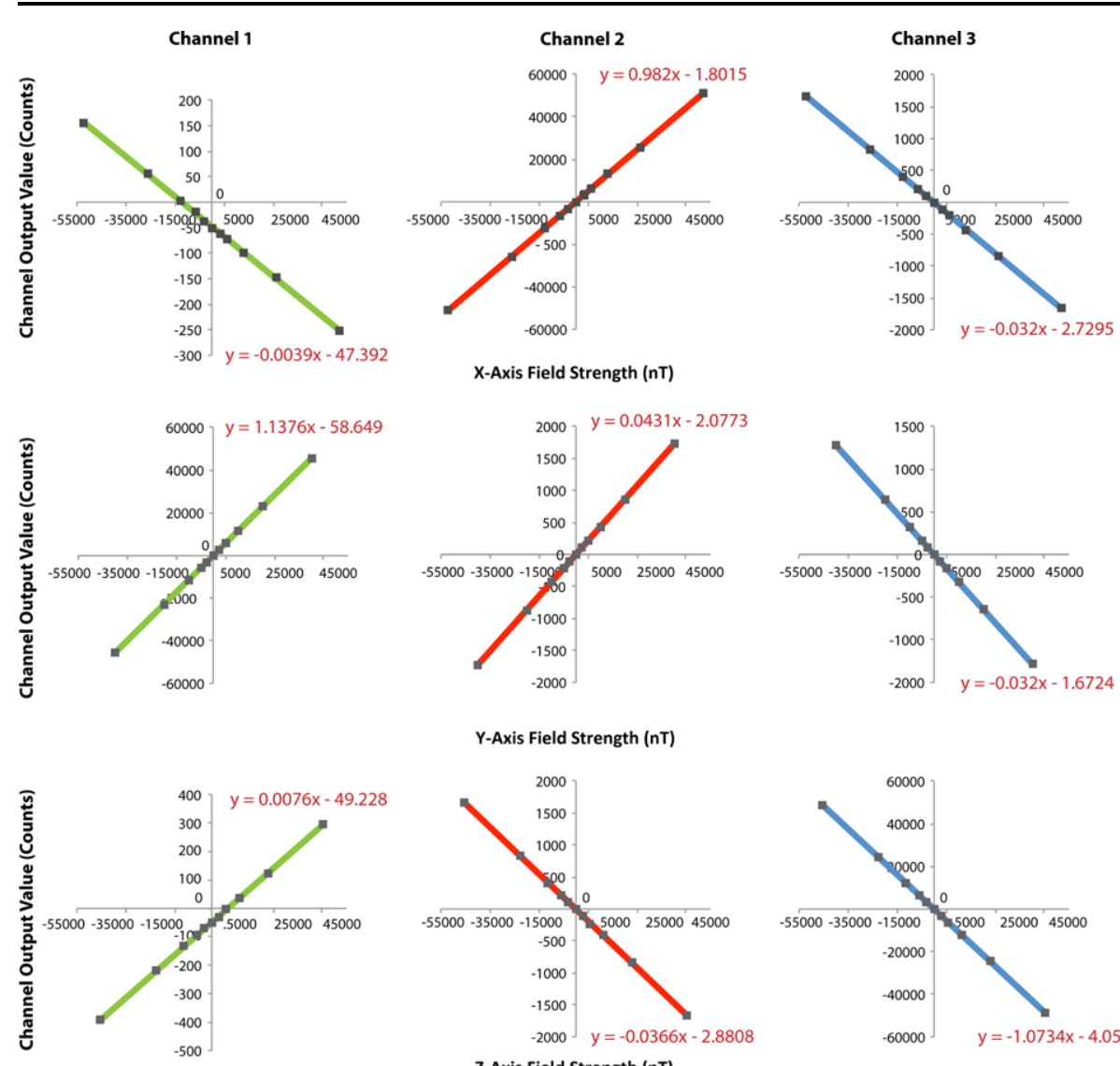

Y-Axis Field Strength (nT)
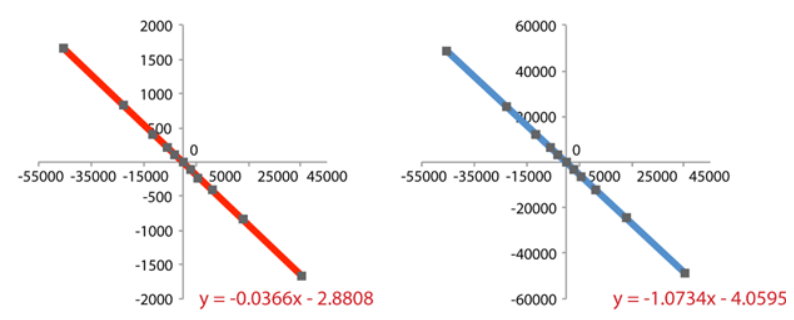

Fig. 39 Calibrated data for the DICE 3-axis magneto-restive SciMag

\section{Launch and On-orbit Functionality}

\subsection{Launch and Early Operations}

The DICE spacecraft (see Fig. 40) were successfully launched as part of the NASA ELaNa III program as a secondary payload out of Vandenberg AFB on the Joint Polar Satellite System NPOESS Preparatory Project launch on October 28, 2011 (see Fig. 41). The orbit insertion information is shown in Table 6.

The two DICE sensor-sats, housed jointly in a 3U P-POD, were launched unpowered. Upon reaching orbit, Farkle was ejected first with Yahtzee directly in pursuit. The two were ejected with a few $\mathrm{cm} / \mathrm{s}$ of relative velocity to create repeating close and distant proximity trailing measurement cases throughout the life of the mission. Following ejection from the P-POD, both DICE spacecraft powered on and entered Safe mode. An approximate 50minute timer, which only engages on the first on-orbit power on and is masked on any subsequent on-orbit power cycles, was also initiated at this point. Following the 50-minute timer delay, the main release mechanism was activated, firing miniature TiNi Frangibolts and deploying the communication antenna and LP booms. The EFP booms were not deployed at 
Fig. 40 The DICE sensor-sats, Farkle and Yahtzee, prior to integration into the launch P-POD

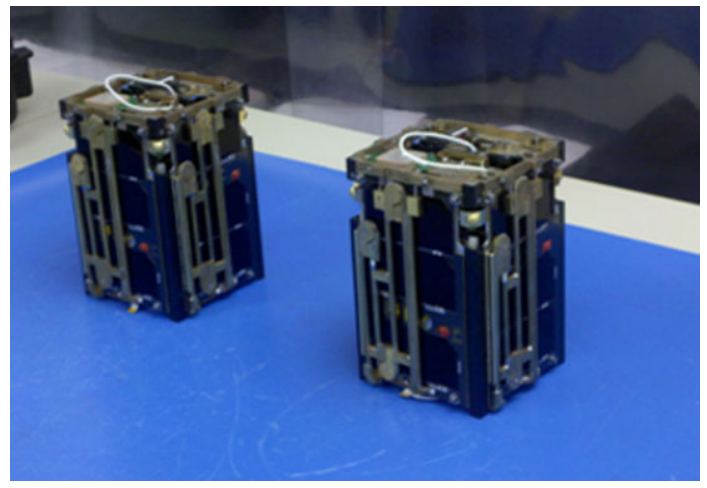

Fig. 41 The DICE spacecraft were launched as secondary payloads on the NASA ELaNa III program

Table 6 DICE spacecraft orbit insertion parameters

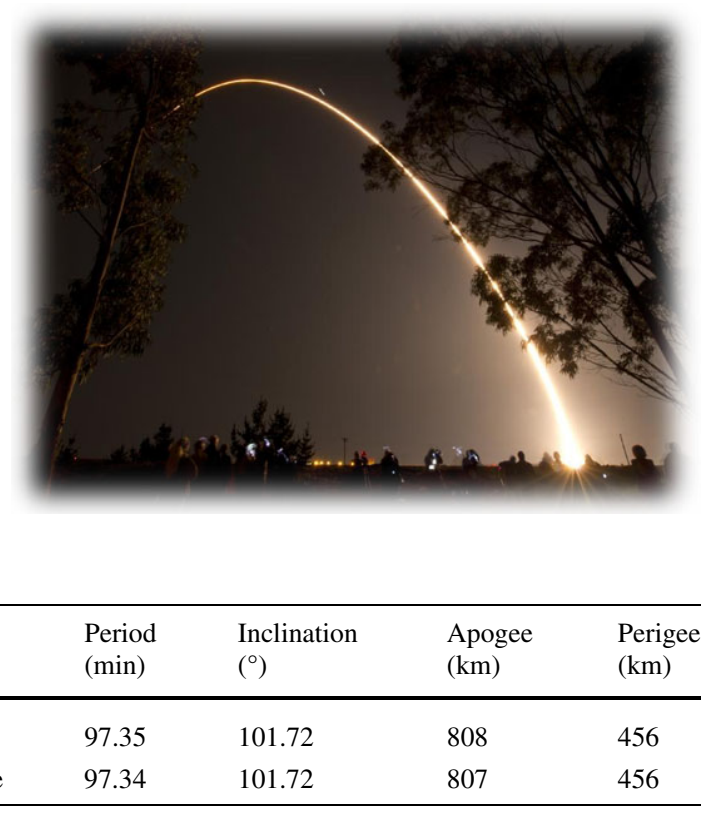

this time. In addition to the structural containment provided by the P-POD, the launch of the CubeSats in an unpowered state followed by the ejection initiated 50-minute timer were key steps in ensuring that the secondary CubeSat payloads did not interact or interfere with the primary launch vehicle payload(s). Additionally, as there were multiple CubeSats launched on the ELaNa III program, the post ejection mechanism deployment delay process helped ensure that each of the ELaNa CubeSats were separated sufficiently before beginning fully deployed operations.

The early orbital events of powering on, followed by the firing of the main mechanism and resultant antenna and LP boom deployment for Yahtzee, are portrayed in the HK parameters shown in Fig. 42. The DICE HK is nominally sampled at $0.1 \mathrm{~Hz}$. The system voltages are plotted in panel a; yVolt, xVolt, and zVolt are solar array input voltage levels. The yVolt line is used for external power via a USB hub during ground testing and is not connected on orbit. The batt1Volt line is the battery bus voltage level and represents the charged state of 

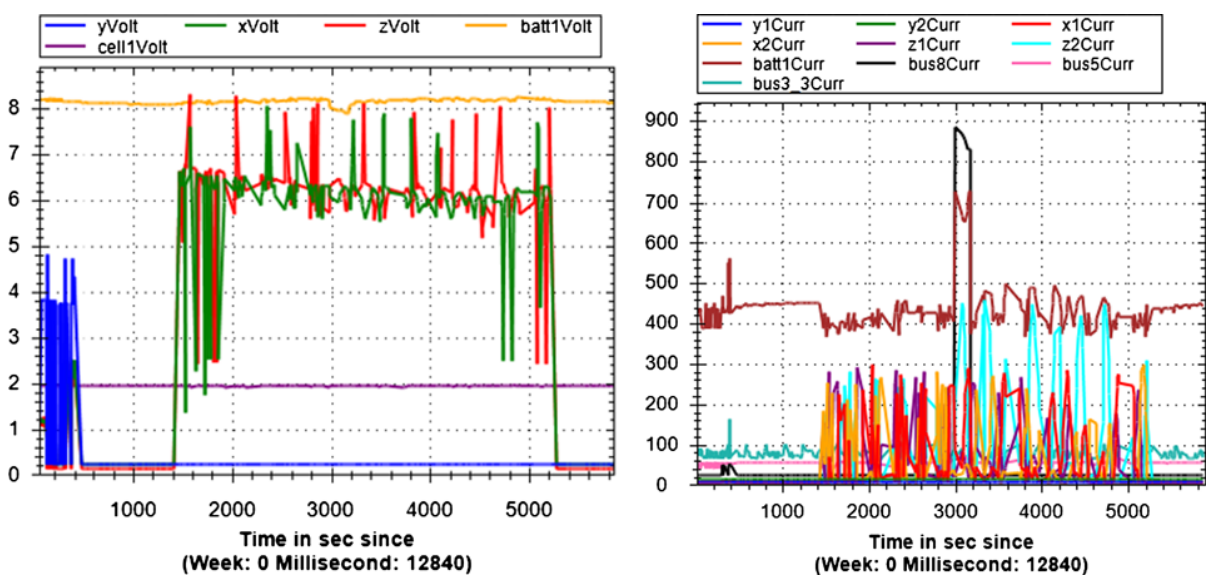

Fig. 42 DICE sensor-sat housekeeping telemetry following launch and ejection into orbit from the PPOD

the batteries. The cell1 Volt line is a battery cell health diagnostic. The system currents are plotted in panel b; y1 and y2Curr, $\mathrm{x} 1$ and $\mathrm{x} 2 \mathrm{Curr}$, and $\mathrm{z} 1$ and z2Curr are solar array input current levels. The yCurr line is used for external power via a USB hub during ground testing and is not connected on orbit. The batt1Curr line accounts for the total flow of current (both into and out of) the batteries. The bus8Curr, buss5Curr, and bus3_3Curr line is the current flow from the batteries to the three main power busses in the spacecraft. Time on is given in relative seconds on the abscissa.

The initial data at the far left in both panels a and b is "left over" in the on-board memory from ground testing. It was intentionally kept in the on-board memory for a clear marker of the power on point following orbit insertion. It ends at approximately 400 seconds. That short period is then followed by voltage and currents captured after ejection from the P-POD and power on of the Yahtzee sensor-sat. The DICE spacecraft were inserted into orbit during the last minutes of an eclipse condition. Shortly thereafter Yahtzee had its first view of the sun, as shown by the sudden increase in solar panel voltages and currents. The dip in the batt1Volt, and the associated spikes in the bus8_Curr and batt1Curr lines at approximately 3300 seconds, are then responses to the firing of the Yahtzee antenna and Langmuir boom mechanism release actuator. The solar panel current data clearly demonstrate a change in spacecraft attitude tumbling pattern from before to after the deployment of the antenna and LP booms. Review of the ADCS data following orbit insertion reveals that the spacecraft were released at a tumble rate of tens of degrees per second and that those rates then slowed following the deployment of the booms. The Farkle power on and initial deployment sequence was very similar to that shown for Yahtzee in Fig. 42.

The first uplink and downlink communications with the DICE spacecraft occurred approximately four days following orbit insertion. Contacts from WFF were attempted directly following orbit insertion but there were no identified responses. It took nearly four days for the government-furnished Two-Line Element sets (TLEs) to mature to the point that our team could correctly point the narrow, high gain Wallops UHF beam onto the DICE spacecraft and obtain a downlink response to an uplink command. TLEs are a data format used to convey sets of orbital elements that describe the orbits of Earth-orbiting satellites. These TLEs are used by ground station systems to predict a satellite's location at any given time. This allows dish-tracking to occur, which is necessary for pencil-beam style dish systems like the WFF communication system. 

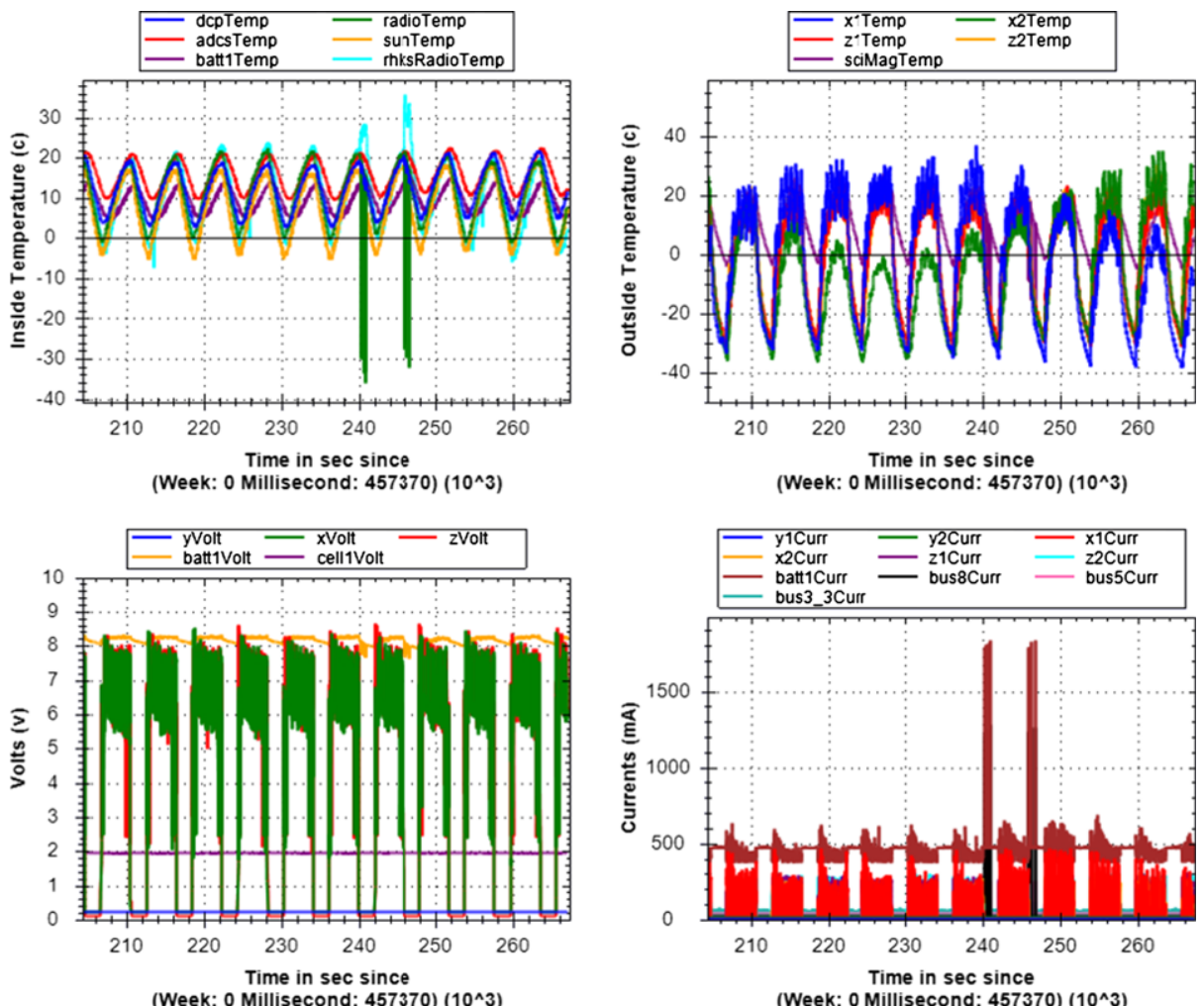

Fig. 43 Orbital DICE housekeeping data

There was significant confusion as to the identities versus published TLEs of the multiple ELaNa III spacecraft following orbit insertion. It took approximately 14 days following launch to clearly distinguish independent TLEs for each of the DICE and the other ELaNa III program spacecraft. Within two weeks following launch, both DICE spacecraft were put into Standby mode and the spacecraft were detumbled to a few degrees per second per axis rotation rates. At this point, both spacecraft began nominal on-orbit maintenance and communication operations.

\subsection{Nominal Operations}

Both DICE spacecraft have been operating on orbit since October 2011. Orbital elements have been refined and tracking and communications ( $9.6 \mathrm{~Kb} / \mathrm{s}$ uplink, $3 \mathrm{Mb} / \mathrm{s}$ downlink) with both spacecraft out of WFF and SRI have occurred regularly during that period. All subsystems have demonstrated operational functionality. The original DICE baseline on-orbit mission duration was planned for 90 days, however it is expected that the DICE mission can operate for an additional three years, limited by battery degradation due to long-term discharge/charge cycling.

\subsubsection{Spacecraft Status}

Figure 43 provides a snapshot of a typical DICE spacecraft condition. It portrays system HK temperatures as shown in the two top plots and the bottom plots portray system voltage 
Fig. 44 DICE sun sensor measurements demonstrating on-orbit average pointing error knowledge of $<0.3^{\circ}$

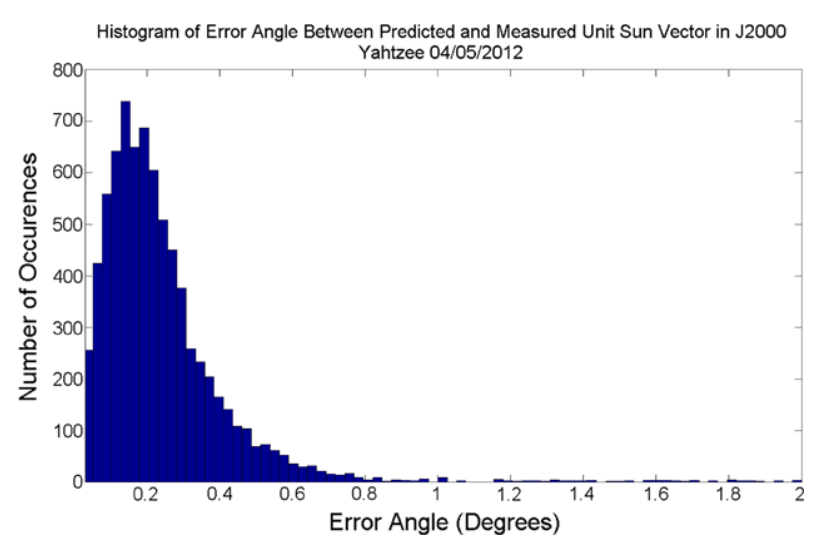

and current readings. The displayed data is from Farkle for the beginning of May 2012. The total time covered is approximately 11 orbits.

As can be seen from the voltage plots in the bottom left panel, the eclipse period is relatively short for this season of the mission (see Fig. 43). At the beginning of each orbit, the solar panel voltages and currents briefly reach maximum values while charging the batteries and powering the spacecraft. As such, the batteries are maintained in a charged state for a good portion of each orbit while the spacecraft is power positive.

As shown in the top left plot of Fig. 43, the Farkle sensor-sat internal temperatures (science board, radio and radio board, ADCS board, primary battery board, sun sensor board) swing over a total range of $\sim 15{ }^{\circ} \mathrm{C}$ during a full orbit, and are biased at approximately $12{ }^{\circ} \mathrm{C}$. This is very benign temperature regime for electronic and battery operation. The larger swing temperatures in the top right plot are for the solar panels which are thermally isolated from the rest of the structure and can easily handle such wide temperature swings. The solar panel temperature patterns vary from orbit to orbit for the panels in accordance with the very slow tumble of the spacecraft that gradually orients different spacecraft panel faces with the solar vector one ach orbit. The spikes in temperature and current (most notable in the top left and bottom right plots) for orbits 7 and 8 are during times of downlink transmission when the radio is in high power mode.

The DICE spacecraft use ADCS magnetometer and sun sensor measurements to control the orientation and spin control of the spacecraft. The sun sensor is key to controlling the spin rate and spacecraft spin axis orientation. Sun sensor data is also critical to performing highly accurate science data analysis. The DICE sun sensors have performed very well on orbit, exceeding expectations. Figure 44 shows the Yahtzee ADCS sun sensor measurement performance as determined from the first few months of operation. The sun sensor is providing on-orbit accuracies of around $0.15^{\circ}$, which is far better than the predicted levels of $\sim 0.7^{\circ}$.

Typical orbit to orbit and daily temperature variations for the spacecraft components were reviewed in Fig. 43. It was seen that the low thermal mass allows for the spacecraft temperatures, especially the solar panels, to vary noticeably during relatively short periods. This level of temperature fluctuation was expected and was part of the driver for the extensive prelaunch instrument calibration effort. Figure 45a depicts the slowly changing thermal bias of the spacecraft for longer, seasonal periods. The data is from Farkle and covers the spring and summer months of 2012 . Figure $45 \mathrm{~b}$ presents the associated eclipse period fraction. In this figure, it is evident that for reasonably long periods of time the DICE spacecraft are in a dawn to dusk sun synchronous orbit with no eclipse. Comparing Fig. 45a and Fig. 45b clearly demonstrates, and is as expected, that eclipse duration has a significant effect on 


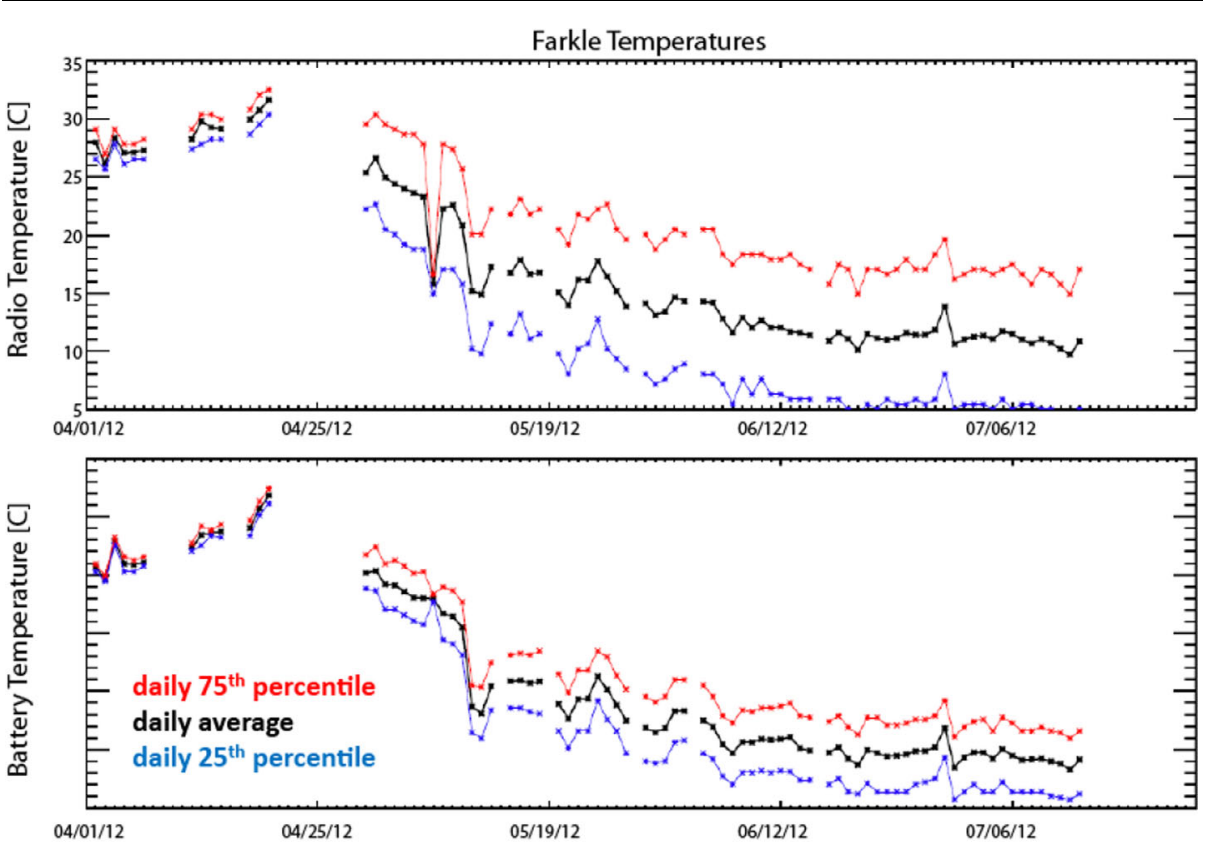

(a)

DICE Eclipse Fraction 4/1/12 to $7 / 25 / 12$

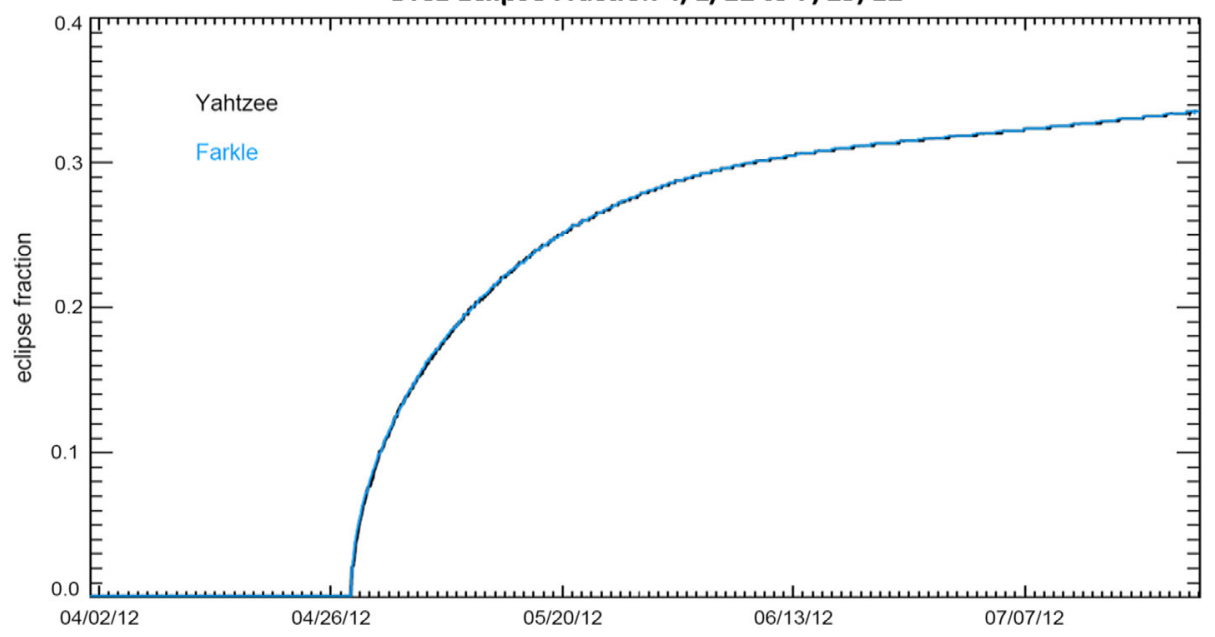

(b)

Fig. 45 DICE orbital temperature variations during the spring and summer of 2012 (a) Farkle spacecraft; (b) an accompanying eclipse period plot; the missing data during April 2012 is due to GS upgrade activities, prohibiting communication

the spacecraft bias temperature. However, even during the high eclipse ratio periods, the bias temperature of the internal spacecraft components remains at a very desirable operating level. The missing data during April 2012 is due to WFF GS upgrade activities during which time communications with DICE were postponed. 


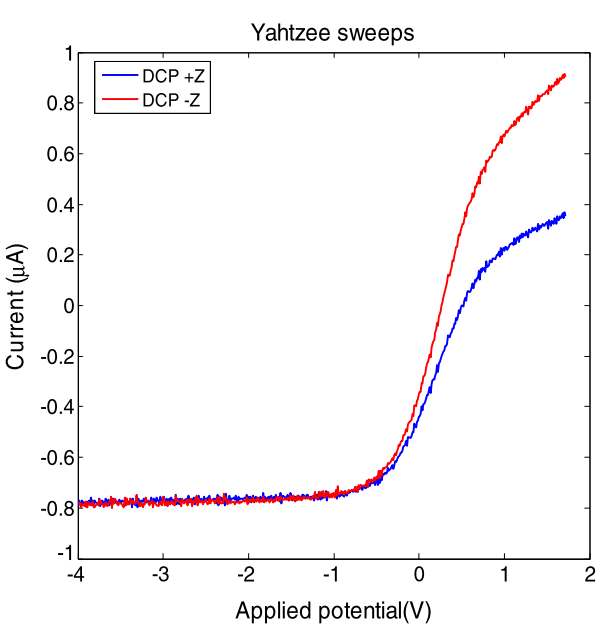

(a)

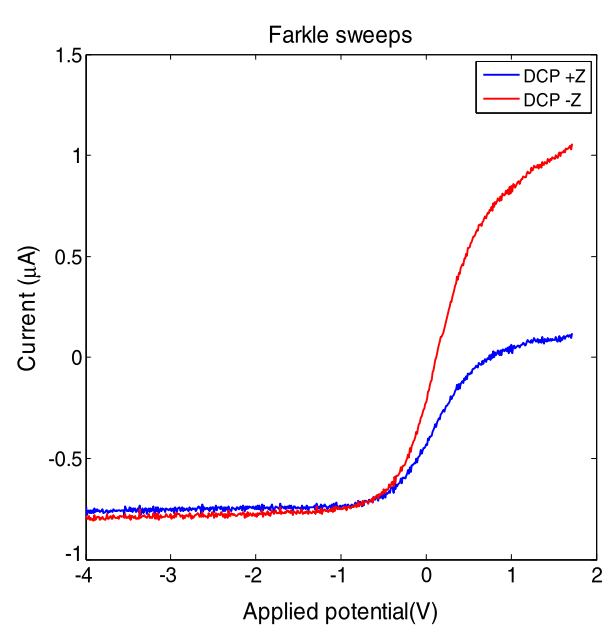

(b)

Fig. 46 On-orbit $I-V$ curves generated by the DICE sensor-sat LPs: (a) Yahtzee sweeps; (b) Farkle sweeps

\subsubsection{Instrumentation Status}

Figure 46 shows on-orbit measurements made by the Yahtzee (panel a) and Farkle (panel b) LPs. These plotted data are from the intermittent sweeps which occur every $120 \mathrm{~s}$ and cover a voltage range from -4 to +1.7 VDC. The shapes of these raw low noise $I-V$ curves follow the projected responses and demonstrate that the LPs are operating as predicted. The difference in magnitude for the responses from the LPs on the same sensor-sat highlight that in general one of the LP sensors will be positioned more fully in the spacecraft velocity direction than the other. Thus, the higher current curves belong to the sensors that are in full or partial RAM, while the lower current curves belong to the sensors that are in a partial wake.

Figure 47 compares multi-orbit Farkle LP data from May 29th, 2012 to outputs of three ionospheric weather models; namely the Ionospheric Reference Model (IRI), ASTRA IDA4D model, and the USU Global Assimilation of Ionospheric Measurements (GAIM) model. IRI is a climatology model, while IDA4D and GAIM are nowcast assimilative models that update on a regular basis, and are forced by ingested real-time observations. USU GAIM used the Ionospheric Forecast Model (IFM) output as the initial background ionosphere, while ASTRA IDA4D used IRI 2007. Each model was run with a 15 minute time step. USU GAIM had a uniform grid of $2.5^{\circ}$ in latitude and $15^{\circ}(\sim 1$ hour) in longitude, whereas ASTRA IDA4D used a non-uniform grid (higher grid density over continental areas). While both models assimilated GPS TEC measurements, IDA4D also assimilated COSMIC occultation data. Due to spinning of the DICE sensor-sats, and as emphasized in Fig. 46, the LPs move in and out of the spacecraft wake. Therefore in order to get one density value for the data in Fig. 47, we chose the maximum ion current from the two probes and then ran a smoothing filter. The fixed bias DICE LP data was normalized to the IRI data for the comparisons. The DICE LP measurements exhibit general agreement with the established ionospheric model predictions, while also demonstrating fine structure detail.

Figure 48 is a comparison of simultaneous measurements from both Yahtzee and Farkle and IRI and IDA4D model outputs for June 29th, 2012. The top panel shows the Yahtzee and Farkle measurements against the co-located model outputs. The bottom panel provides 


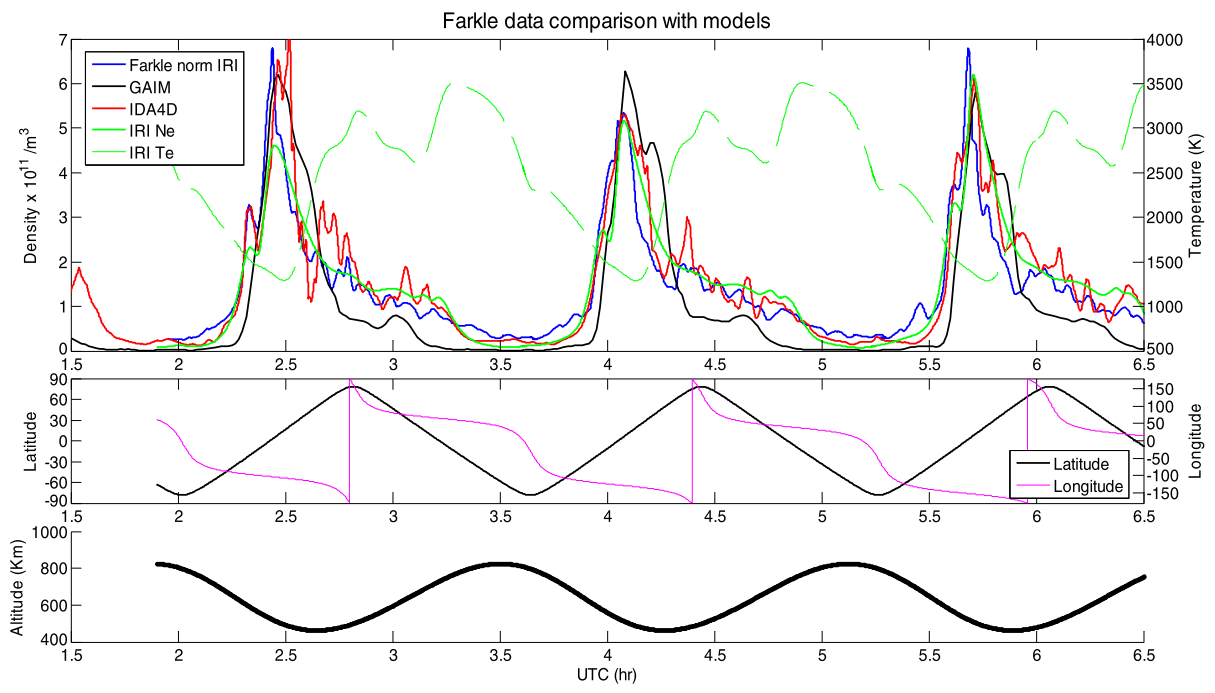

Fig. 47 Comparison of Farkle LP data with the IRI, IDA4D, and USU GAIM models for May 26th, 2012; times are in UT

a global reference of the DICE sensor-sat measurement tracks on top of IDA4D generated ionospheric density outputs for $500 \mathrm{~km}$. In making these plots, the two ion density DICE LP measurements for each spacecraft were averaged and then low-pass filtered and normalized to IDA4D model outputs. Once again, the DICE LP observations agrees very well with the model outputs, while not unexpectedly providing greater resolution to the overall structure. These plots also help emphasize how simultaneous measurements of spacecraft in trailing orbits can help distinguish between temporal and spatial attributes of the ionosphere. Very similar structures are seen by both spacecraft as they go through nearly identical portions of the ionosphere. However, there is also enough orbital separation (nearly $1 / 2$ an orbit for this time of the mission), that they also fly through different portions of the ionosphere at dissimilar times and provide perspective on global spatial structure and continuity.

Next, Fig. 49 investigates co-incident Yahtzee SciMag and ADCS magnetometer measurements acquired June 3, 2012. Figure 49 panel a demonstrates the very good, high level agreement in the measurements from the two sensors. As they should, the post-processed signals from both sensors lay directly on top of one another (see processing details below). The slow tumble/spin of the spacecraft is also evident in the data. Panel $b$ is then is a close up examination of a portion of the measurements, establishing the much lower noise floor of the SciMag measurements. The $<10$ nT noise floor for the SciMag measurement enables detection and monitoring of geophysical disturbances and perturbations. This is clearly seen in Fig. 50 which is a plot of geomagnetic activity captured by the Farkle SciMag on May 22,2012 . The activity is due to field aligned currents (FACs).

Figure 50 is a plot of geomagnetic activity captured by the Farkle SciMag on May 22, 2012 that is due to field aligned currents (FACs).

Figure 51 is a demonstration of the processing of the data from Fig. 50 to investigate the FAC-induced disturbance. Three AMIE panels, spaced at five minute intervals, correspond to different times of the overall $\mathrm{dB}$ event measured by Farkle, as shown in top panel. The DICE orbit path is superposed on AMIE plots, with red triangles showing DICE location at the specific UT times. AMIE reveals that the DICE measured dB event corresponds to a 

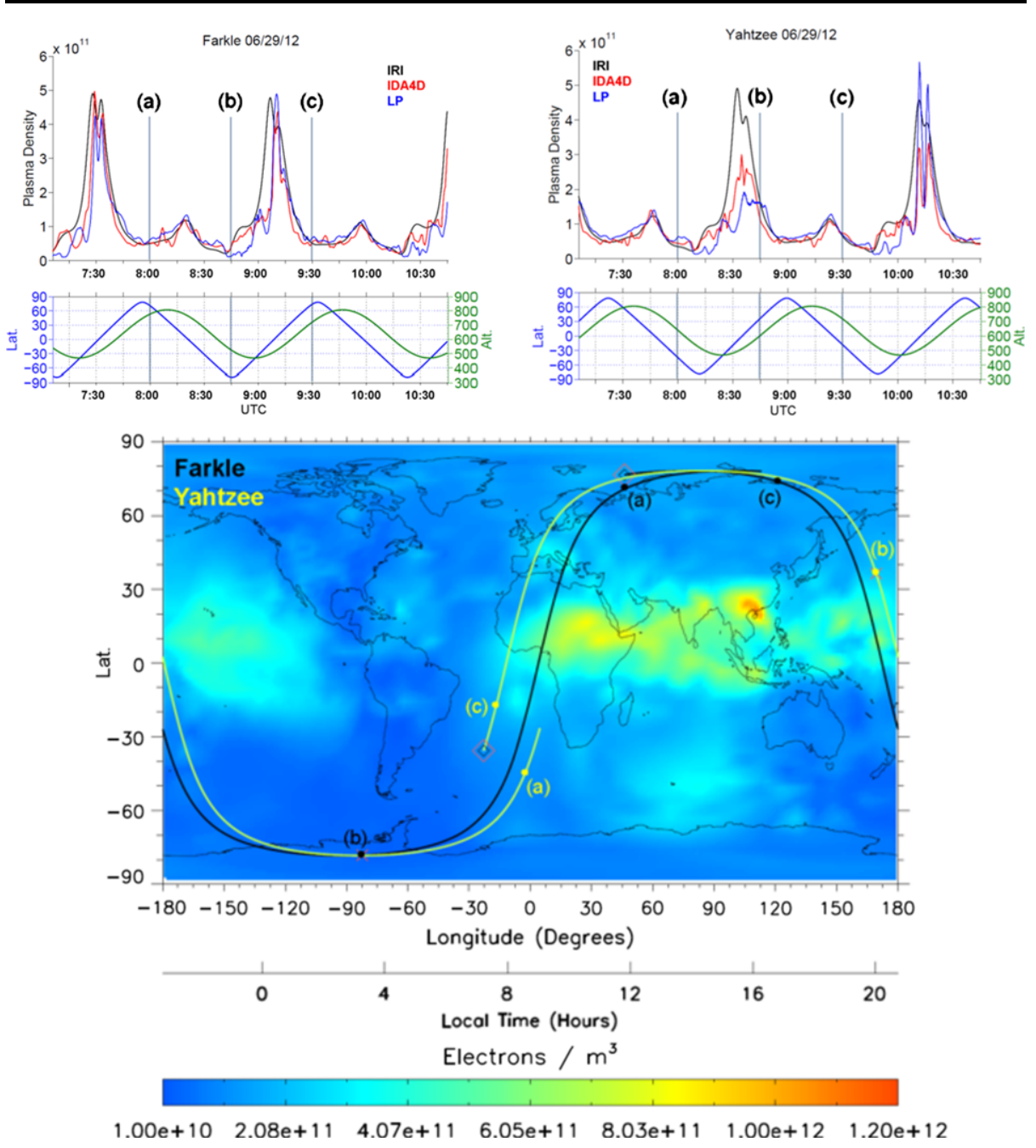

Fig. 48 Comparison of simultaneous Farkle (top left) and Yahtzee (top right) LP measurements versus IRI and IDA4D model outputs for June 29th, 2012. The bottom panel shows a global IDA4D plasma density map for $500 \mathrm{~km}$, with the DICE orbital tracks displayed for reference; the letter annotations in the bottom panel correspond to the correspondingly marked times in the upper two plots

region of enhanced FACs in the cusp region. The DICE SciMag data was processed in the following steps to identify small (1\%) magnetic perturbations caused by the FACs.

1. Ground derived SciMag measurement calibration coefficients were fine-tuned by comparison with on-orbit attitude magnetometer measurement (a and b in Fig. 51).

2. The SciMag data was then filtered to remove periodic spikes originating from periodic spacecraft generated noise from the EPS and other digital systems (panels b and c).

3. A high-pass filter was then used to remove signals due to gradual attitude variations leaving the higher frequency residual, $\mathrm{dB}$, components (panel d). Note that this method of de-spinning the magnetometer data (in the spacecraft body frame) is not dependent on the accuracy of attitude determination. 


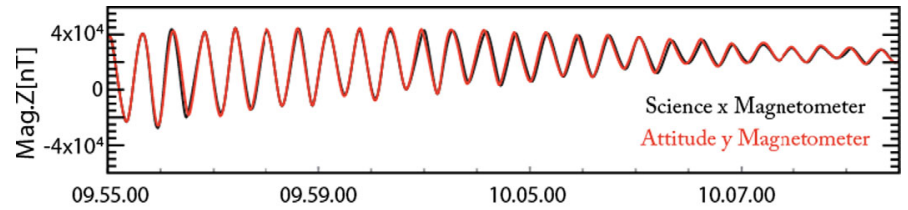

(a)

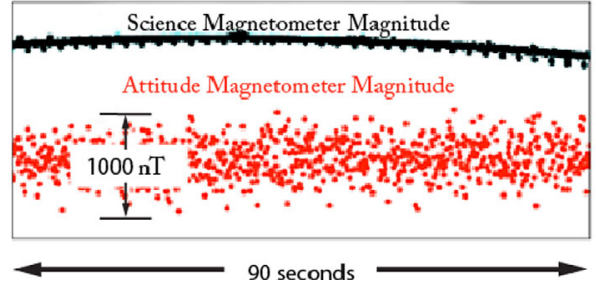

(b)

Fig. 49 (a) Comparison of the DICE ADCS magnetometer and SciMag measurements on June 3, 2012; see Fig. 4 for the axes alignments of the two magnetometers; (b) a close up of the measurements, demonstrating the much lower noise $(\sim 5-10 \mathrm{nT})$ associated with the SciMag measurements

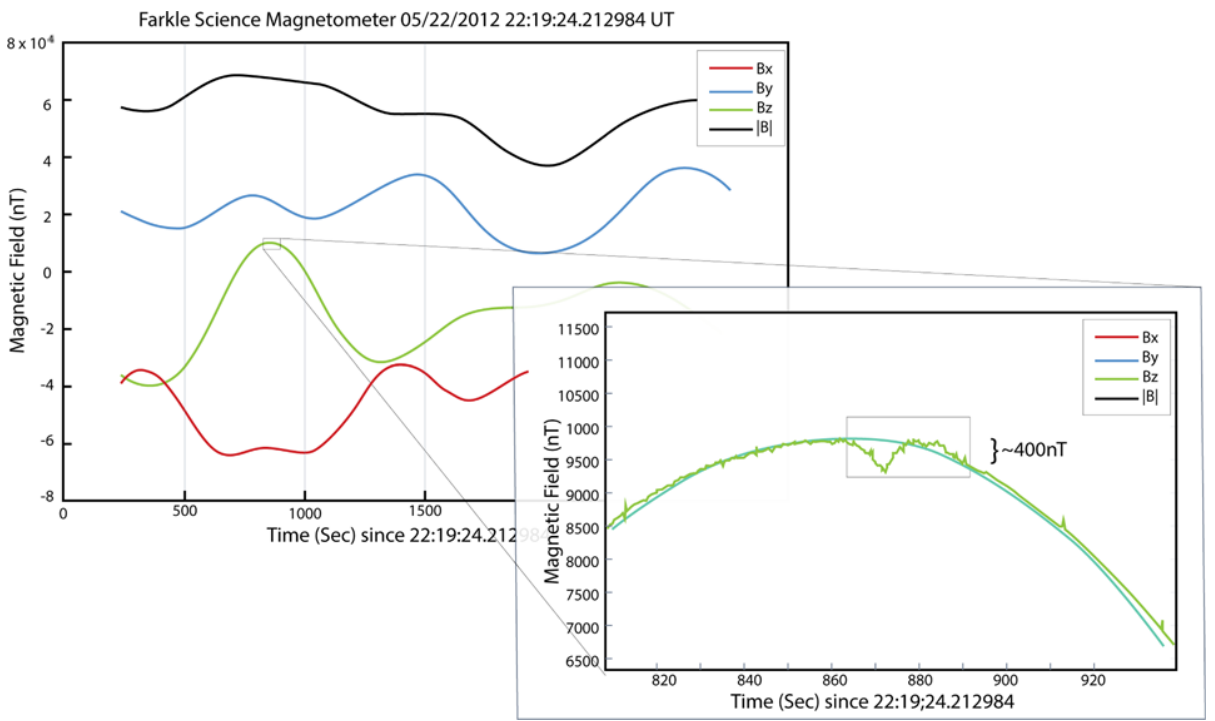

Fig. 50 Geomagnetic disturbance measured by the Farkle SciMag on May 22, 2012

Figure 52 is a comparison of the Farkle SciMag measurements from May 22, 2012 versus AIME model outputs. In the figure, AMIE potential plots (black line contours) with downward FACs (color contours) are shown during a northward Bz, strong By event. The Farkle data is shown as the magnitude of the vector magnetic residual from the IGRF model or $\mathrm{dB}$. The intensity of Farkle $\mathrm{dB}$ corresponds well with the configuration of the electromagnetic disturbance along Farkle's orbital track. The strongest portion of the Farkle dB response is coincident with the biggest changes in current as predicted by AMIE. This analysis further highlights the great potential of the Farkle SciMag measurements. 

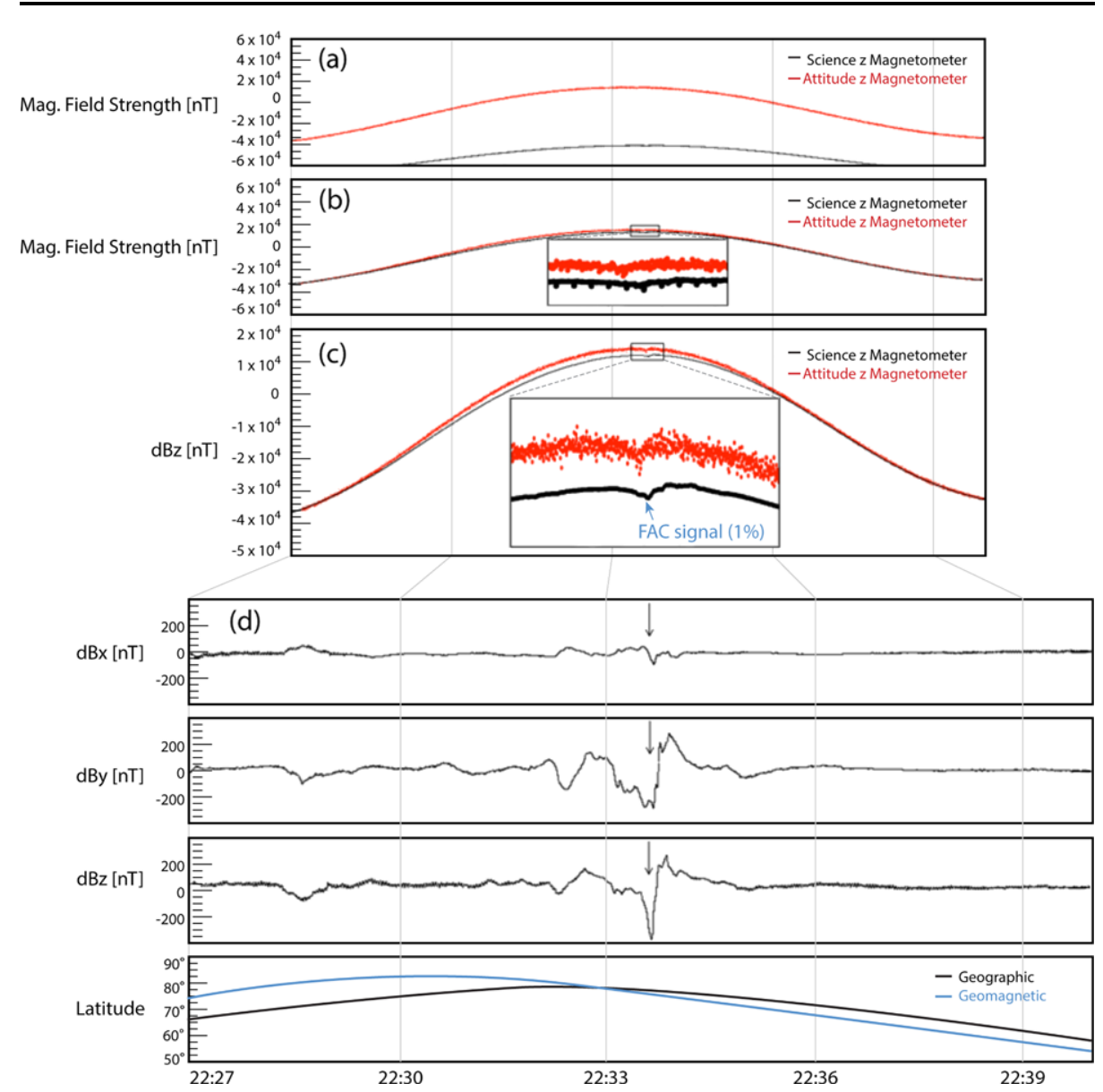

Fig. 51 Analysis of the Farkle SciMag data from May 22, 2012 to investigate the geomagnetic perturbation caused by FACs

The EFP sensors are also acquiring data, however the electric field wire booms have not been deployed as of yet and the electric field sensor spheres continue grounded (stowed configuration) to the spacecraft structures. Deployment of the EFP wire booms would have been a next step in the DICE on-orbit operations process. The on-orbit DICE spacecraft moments of inertia have not been as predicted, resulting in minor axis spinners about the $Z$ axis (long axis of the spacecraft) that have prohibited the deployment of the EFP booms. It appears that the final segments of the UHF booms did not lock into place as expected on orbit and have not provide the added extended mass to create a major axis spinner about the $Z$ axis. If they had, the nominal DICE mission operations process would include a final orientation of the spacecraft $Z$ axis with the geodetic axis (see Fig. 53). Initially, the spacecraft would have been spun up to $\sim 2 \mathrm{~Hz}$ around the $Z$ axis to deploy the booms. The final spin rate, following the $5 \mathrm{~m}$ wire boom deployments, would have been $\sim 0.1 \mathrm{~Hz}$. Electric field measurements would have been made throughout this event to fully characterize the boom deployment process.

A planned nominal spacecraft spin stabilization would have provided two-dimensional measurements of the electric field, and thus allowed a deterministic ground processing re- 


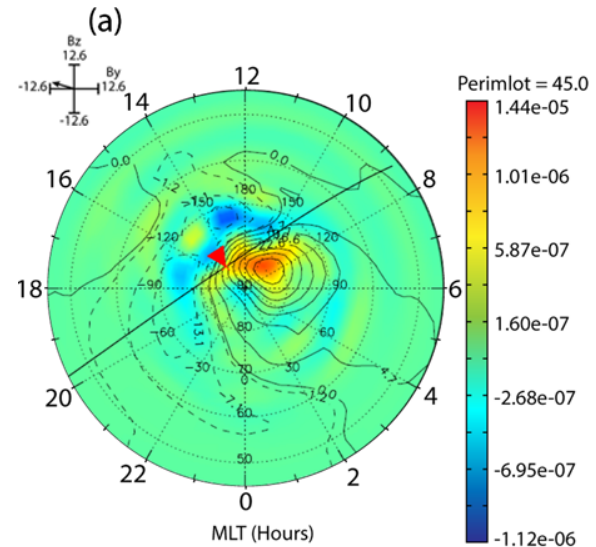

(a)

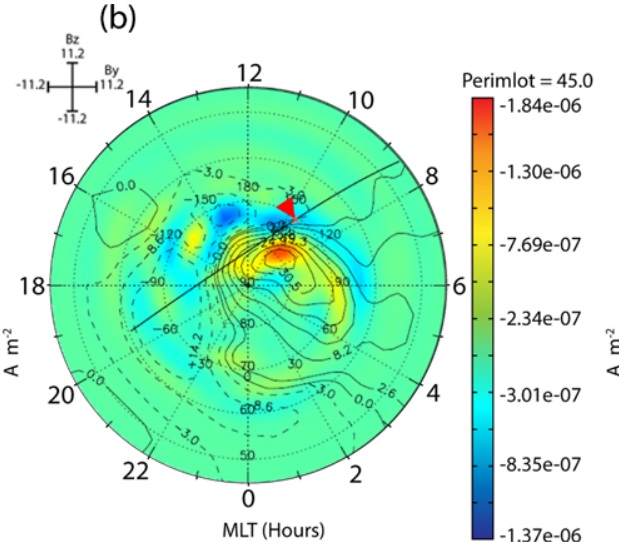

(b)

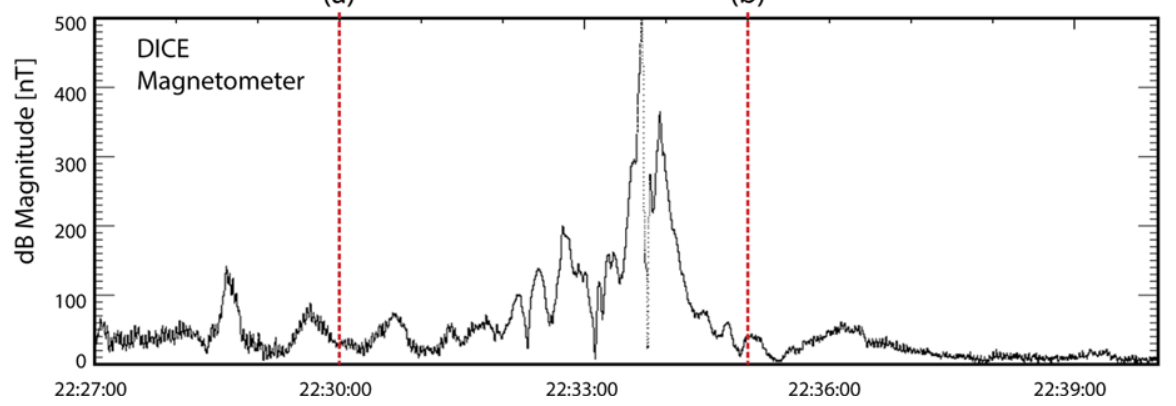

Fig. 52 Comparison of AIME model FAC's versus the Farkle SciMag dB magnitudes for the geomagnetic disturbance observed on May 22, 2012

Fig. 53 DICE sensor-sat orbital orientation in final spin up state

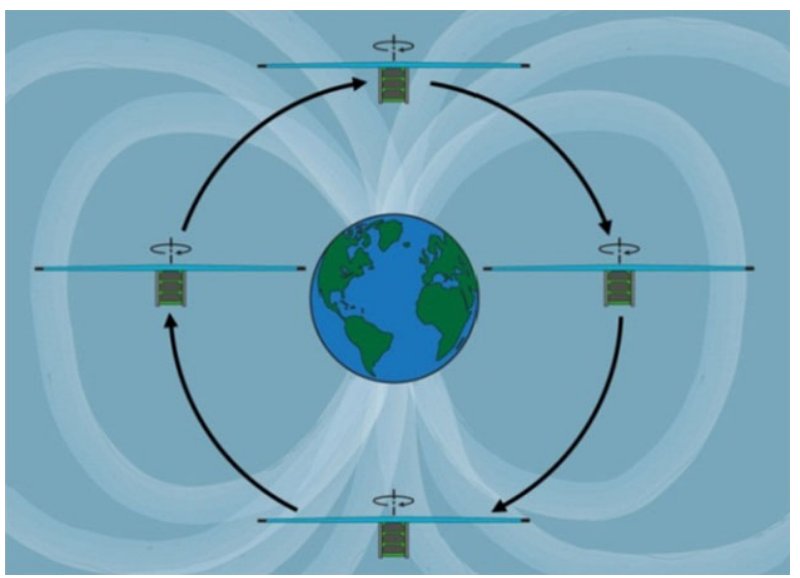

moval of the $\mathbf{V} \times \mathbf{B}$ induced electric field component that is superimposed upon the target environmental electric field due to spacecraft movement through the geomagnetic field. The worst case $\mathbf{V} \times \mathbf{B}$ induced electric field component is expected to be on the order of $350 \mathrm{mV} / \mathrm{m}$, while the quiet time environmental EFP measurement capability is expected to be on the order of $0.5 \mathrm{mV} / \mathrm{m}$. Each DICE spacecraft were designed to spin with the space- 
Fig. 54 The DICE sensor-sats have been gradually separating since mission launch

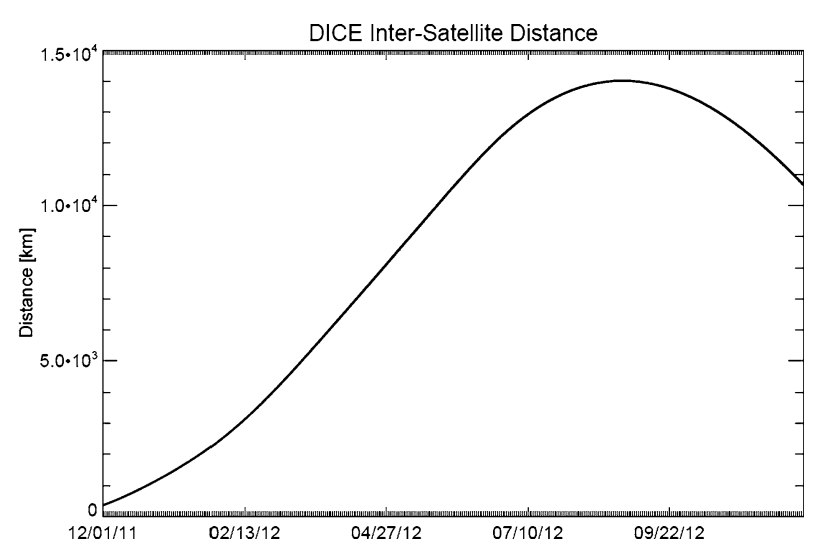

craft $Z$ axis aligned to $\leq 5^{\circ}(1 \sigma)$ of the inertial $\mathbf{J} 2000 z$-axis. As discussed, this orbital orientation facilitates the measurement of the electric field component orthogonal to the geomagnetic field over a majority of the orbit but additionally, this orbital orientation ensures a purely spherical projection of the DCP sphere into the spacecraft ram direction with only minor effect from the cylindrical boom at high latitudes. It is expected that post-processing would determine spacecraft attitude to much less than $1^{\circ}(1 \sigma, 3$-axis).

The progression of the relative separation of the DICE sensor-sats is shown in Fig. 54. This slow separation (and approach) has allowed for study of ionospheric events with varying temporal and spatial characteristics. This process will continue over the life of DICE. Additionally, the DICE orbits continue to precess and move in and out of prime SED coverage. The first prime SED science window occurred shortly after launch, with (see Fig. 55) with future windows cycling into DICE's view every seven months.

\subsection{Communications and Mission Operations}

There has been a tremendous improvement in the amount of downlinked DICE data that can be demodulated and decoded by the software defined radio since the inception of the program. The first set of DICE telemetry was demodulated and decoded at $\sim$ a $10 \%$ success level. However, at the time of this writing, that success level has increased to a consistent 90 $100 \%$. The change in successful demodulation and decoding and final ingestion of the data in to the DC is due to a number of key changes in the hardware and software components of the GS's software defined radio, as well as changes in operational communication bands. The uplink communications have been fully functional at a $100 \%$ success level since launch. The total amount of HK, ADCS, and science data demodulated from both satellites since launch has been 8.1 GByte.

DICE downlink communications began centered at $465 \mathrm{MHz}$. Site surveys at WFF and SRI prior to launch indicated that the $468 \mathrm{MHz}$ band was spectrally cleaner, but a miscommunication during the formal frequency operation request process resulted in a $465 \mathrm{MHz}$ approval. However, DICE was also formally recognized as being able to operate over the entire 460-470 MHz space to earth spectrum during the frequency operation request process; thus, given appropriate approval, DICE could move to other center bands of operation in the $460-470 \mathrm{MHz}$ spectrum if needed. It was quickly determined that a change would be needed. As the 460-470 MHz space to earth spectrum is a secondary user role, DICE downlink transmissions were consistently competing with noise and chatter due to local ground communication (e.g., police and government activities, pager systems, etc.). It was quickly 
Fig. 55 The DICE prime-time science windows are between 1400-1700 LT; (a) definition of the first window; (b) DICE sensor-sats will cycle through the prime-time science windows $\sim$ every 7 months

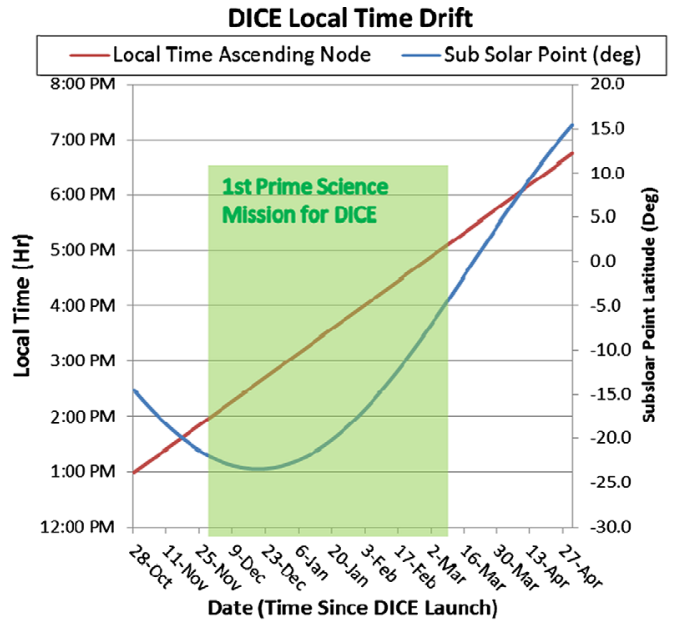

(a)

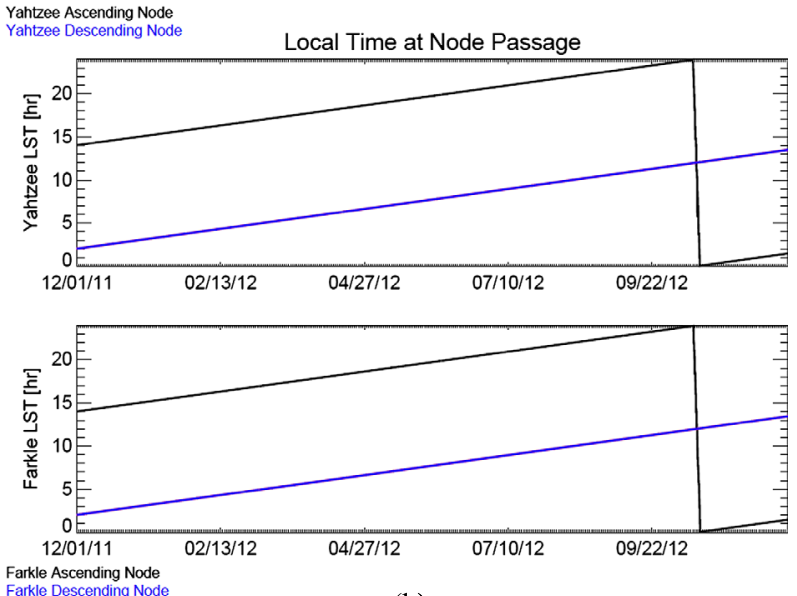

(b)

determined through daily operations that centering around $468 \mathrm{MHz}$ would give DICE a much quieter, although not completely silent, center band of operation for its approximate $\pm 1.5 \mathrm{MHz}$ of required bandwidth.

A formal request was made to change the DICE center band of operation to $468 \mathrm{MHz}$. However, in addition to this operational change, the DICE team made significant improvements in both the hardware and software aspects of the software defined radio GS. First, in collaboration with RF engineers at WFF, the team developed a very sharply filtered lownoise amplifier for $468 \mathrm{MHz}$ operation to go on the front of the USRP2 receiver input. The prior design was a much wider band implementation. Second, the DICE software defined radio team developed new signal processing techniques to remove a large portion of the interference, while still allowing for detection, demodulation, and decoding of the resultant DICE data. An example of this "cleaning" process is shown in Fig. 56.

The formal approval to operate at $468 \mathrm{MHz}$ occurred in May 2012. The DICE spacecraft were commanded to downlink at this frequency, the WFF filter and low-noise amplifier were put in place, and the new software-defined radio signal processing techniques were included 
Fig. 56 The DICE team developed signal processing code to scrub the noisy data and remove the competing local chatter that interferes with the desired DICE spectrum and the spectrogram plots are centered at $465 \mathrm{MHz}$; the top two plots are running spectrograms with time in seconds and in the bottom plot, the green spectrum profile is what is left after the "cleaning" process has occurred (correlates with middle plot) - the blue spectrum profile in the bottom plot correlates with the top plot
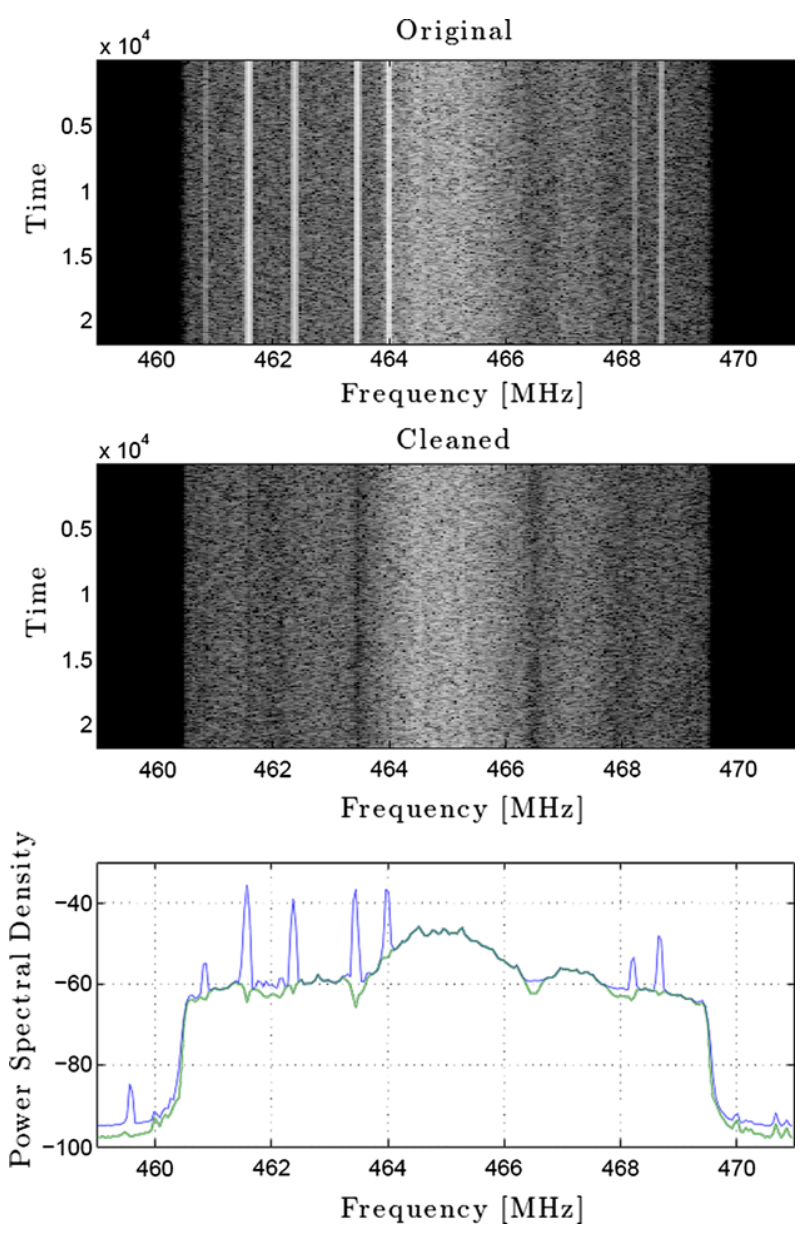

in the code set of the GS. A combination of these pieces, as well as other refinements of the software-defined radio code, has increased the DICE data capture level from a mere $10 \%$ to nearly $100 \%$ for all downlinks. Stored I/Q raw data captured by the software-defined radio has allowed post-processing of data from early in the mission. At the time of this writing, nearly 20 Terabytes of raw data has been archived at USU/SDL facilities. This has allowed the team to achieve this nearly $100 \%$ data capture level from overpasses at the first of the mission. All of the ground-breaking work done with the communications system has allowed the program to downlink $\sim 65$ Gbits of data from the two sensor sats. This data volume is significantly more than that of a standard CubeSat. This opens up a door to science-intensive missions achieved with these miniature sensor sat platforms. A thorough review of the signal processing development for DICE communications can be found in Gunther et al. (2013).

Due to high levels of tracking performance from the GSs at WFF and SRI, and the nearly isotropic DICE spacecraft antenna pattern, DICE earth to space and space to earth communications quite often occur from horizon to horizon $\left(0\right.$ to $\left.0^{\circ}\right)$ at uplink and downlink paths at ranges $>3000 \mathrm{~km}$. When operating in a 24/7 mini-campaign operation mode with both WFF and SRI GSs active, $\sim 90$ minutes. 


\section{Conclusions}

The DICE mission has demonstrated a number of first ever science measurements from a CubeSat, including multi-platform measurements of Langmuir Probe and Science Magnetometer measurements. In this article, we have shown initial on-orbit functionality of these sensors, including observation of FACS from the body-mounted SciMag. Forthcoming publications will more thoroughly investigate the on-orbit calibration and science performance of the DICE LP and SciMag. In the near future, it is expected that the DICE team will deploy the EFP booms. This activity will demonstrate the ability to make multi-meter doubleprobe measurements from a miniature CubeSat platform. These successful demonstrations of science grade measurements from a CubeSat are notable first step in a pathfinder process to low-cost, multi-point CubeSat constellation observations of the Sun-to-Earth system. As discussed, future expectations are for multiple copies of a DICE sensor-sat to be inserted jointly into orbit, enabling networked measurements the state of Earth's upper atmosphere.

DICE has also been revolutionary in opening up a number of groundbreaking technologies to the CubeSat community. Most notably has been the development and demonstration of a multi-Mb/s downlink radio for use on regularly licensed bands. This development includes not only the design and implementation of the high-speed space to ground CadetU radio, but also the tools, facilities, and infrastructure to provide the ground tracking, communication link closure, and data acquisition and management on the ground. This has come in large part to the teaming with L-3 Communications and NASA WFF. DICE has also promoted and demonstrated a miniature, reliable CubeSat deployment mechanism actuator based on the TiNi SMA technology.

Given the very low budget pathfinder and educational nature of the DICE mission, a number of lessons learned were expected. The DICE team feels that these lessons learned that are worth sharing with the community in hopes of helping push forward the great CubeSatbased space research and operations revolution that is advancing at such an accelerated pace.

\subsection{Programmatic Lessons Learned}

1. Great things can indeed come from humble settings. While the funding allocated to the NSF CubeSat programs is miniscule in comparison to most other government-sponsored space flight programs with similar expectations, the leeway given to the PI, professional, and student teams to implement the mission encourages incredible resourcefulness and efficient use of program resources.

2. Positive collaboration between government, academia, small business, and industry with a set of common goals can be very productive in developing far reaching space mission technologies and processes at very low cost and impact. The DICE team was immensely benefited by collaboration and contributions from L-3 Communications, TiNi Aerospace, ATK, WFF, and SRI. Very high-speed space to earth communications, miniature reliable actuators, and high end software-defined radios for GSs were developed through collaborations and teaming.

3. The support of NASA ELaNa in providing launch services to the CubeSat community is invaluable in the sense that it allows the individual mission teams to focus on spacecraft, sensor, and mission operations development. The coordination of launch integration is a very large task and the removal of that burden from the NSF CubeSat program mission teams has a very positive impact. 


\subsection{Technical Lessons Learned}

1. While the development and assembly of small CubeSats can be much easier and quicker than larger spacecraft, the on-orbit spacecraft operations and management of the downlinked data is equivalent in scope to traditional missions. Once the CubeSats have reached orbit, all semblances of "smallness" disappear. Thus, the approach for management of research and operational CubeSat missions needs to be considered in respect to traditional mission approaches.

2. The engineering challenge of producing well performing science instruments within the technical resource constraints of a CubeSat is every bit as valuable as seeing how big we can make our farthest seeing large telescopes. Pushing the frontiers of technology is invaluable to our future progress and competitiveness.

3. NSF and NASA-sponsored CubeSat programs in general can greatly benefit by using government-requested communication bands and established GS sites at WFF, SRI, and potentially other facilities such as Morehead State. This does not exclude use of the amateur community infrastructure (e.g., use of beacons on CubeSats), but it does encourage regular research and operations classification of future government sponsored CubeSat programs and allows the CubeSat community to take advantage of the great amount of engineering that has already occurred in developing the larger GS facilities.

4. CubeSats should, and will be, the backbone of many future global multi-point measurement missions (and not just for geospace). Pushing from the CubeSat end of the size spectrum has opened up many new paths to low cost, easier access to space for upcoming constellation missions.

Acknowledgements The authors gratefully acknowledge funding provided by NSF (grant numbers\# ATM0838059, AGS-1212381, AGS-1255782) and to the NASA ELaNa III group for launch services. The team would also like to gratefully acknowledge the countless hours of dedicated and passionate effort from the students on the DICE program. They indeed rose to the challenge. Without their energy and consistency, DICE would not have become a reality.

Open Access This article is distributed under the terms of the Creative Commons Attribution License which permits any use, distribution, and reproduction in any medium, provided the original author(s) and the source are credited.

\section{References}

W.E. Amatucci, M.E. Koepke, T.E. Sheridan, M.J. Alport, J.J. Carol III, Self-cleaning Langmuir probe. Rev. Sci. Instrum. 64(5), 1253 (1993)

A. Barjatya, C.M. Swenson, J. Geophys. Res. 111(A10) (2006)

A. Barjatya, C.M. Swenson, D.C. Thompson, K.H. Wright, Data analysis of the floating potential measurement unit aboard the international space station. Rev. Sci. Instrum. 80, 041301 (2009). doi:10.1063/1.3116085

Su. Basu, S. Basu, J.J. Makela, R.E. Sheehan, E. MacKenzie, P. Doherty, J.W. Wright, M.J. Keskinen, D. Pallamraju, L.J. Paxton, F.T. Berkey, J. Geophys. Res. Lett. 32 (2003). doi:10.1029/2004GL021669

G.S. Bust, T.W. Garner, T.L. Gaussiran II, J. Geophys. Res. 109, A11312 (2004). doi:10.1029/ 2003JA010234

J.B. Camp, T.W. Darling, R.E. Brown, Macroscopic variations of surface potentials of conductors. J. Appl. Phys. 469(10), 7126 (1991)

A.J. Coster, J. Foster, P. Erickson, Space weather. GPS World 14(5), 42-49 (2003)

G. Crowley, C. Hackert, R.R. Meier, D.J. Strickland, L.J. Paxton, X. Pi, A. Mannucci, A. Christensen, D. Morrison, G. Bust, R.G. Roble, N. Curtis, G. Wene, J. Geophys. Res. 111, A10S18 (2003)

CubeSat Design Specification, Rev. 12, California State Polytechnic University (2010). http://browncubesat. org/wp-content/uploads/2013/01/Cubesat-Reqs.pdf 
L. David, CubeSats: Tiny Spacecraft, Huge Payoffs. Available from http://www.space.com/308-cubesatstiny-spacecraft-huge-payoffs.html (2004)

G.D. Earle, M.C. Kelley, G. Ganguli, J. Geophys. Res. 94(A11), 15321-15333 (1989)

U. Fahleson, Space Sci. Rev. 7, 238-262 (1967)

J.C. Foster, J. Geophys. Res. 98, 1675 (1993)

J.C. Foster, A.J. Coster, J. Atmos. Sol.-Terr. Phys. 69, 1241-1252 (2007)

J.C. Foster, W. Rideout, Geophys. Res. Lett. 32 (2005)

J.C. Foster, P.J. Erickson, F.D. Lind, W. Rideout, Geophys. Res. Lett. 31, L21803 (2004). doi:10.1029/ 2004GL021271

J. Gunther, T. Moon, C. Swenson, C. Fish, Reliable space-to-earth communication as a secondary service in the 460-470 MHz band. Int. J. Satell. Commun. Netw. (2014, in press)

J.A. Klobuchar, J. Aarons, H.H. Hosseinich, J. Geophys. Res. 73, 7530-7534 (1968)

A.J. Mannucci, B.T. Tsurutani, M.A. Abdu, W.D. Gonzales, A. Komjathy, E. Echer, B.A. IIjima, G. Crowley, D. Anderson, J. Geophys. Res (2007)

N.C. Maynard, Electric field measurements in moderate to high density space plasmas with passive double probes, in Measurement Techniques in Space Plasmas Fields, ed. by R.F. Pfaff, J.E. Borovsky, D.T. Young (American Geophysical Union, Washington, 1998). doi:10.1002/9781118664391.ch2

M. Mendillo, Rev. Geophys. 44 (2006)

NASA, Heliophysics the Solar and Space Physics of a New Era, Recommended Roadmap for Science and Technology 2009-2030 (2009). http://science.nasa.gov/media/medialibrary/2010/03/31/ Heliophysics_Roadmap_2009_tagged-quads.pdf

NASA, Solar and Space Physics: A Science for a Technological Society: The 2013-2022 Decadal Survey in Solar and Space Physics (2012)

S. Skone, M. El-Gizawy, S.M. Shrestha, Radio Sci. 39, RS1S17 (2004). doi:1029/2002RS002884

J.J. Sojka, D. Rice, J.V. Eccles, F.T. Berkey, P. Kintner, W. Denig, Space Weather 2, S10006 (2004)

C.T. Steigies, A. Barjatya, Contamination effects on fixed-bias Langmuir probes. Rev. Sci. Instrum. 83, 113502 (2012). doi:10.1063/1.4764582

D. Vallado, Fundamentals of Astrodynamics and Applications. The Space Technology Library, 2nd edn. (Microcosm, Inc., Hawtkorne, 2001) 